\title{
q-Virasoro Modular Double and 3d Partition Functions
}

\author{
Anton Nedelin ${ }^{1,2}$, Fabrizio Nieri ${ }^{3}$ (D), Maxim Zabzine ${ }^{3}$ \\ 1 Dipartimento di Fisica, Università di Milano-Bicocca, Piazza della Scienza 3, 20126, Milan, Italy \\ 2 INFN, sezione di Milano-Bicocca, 20126, Milan, Italy. E-mail: anton.nedelin@ unimib.it \\ 3 Department of Physics and Astronomy, Uppsala University, Box 516, 75120, Uppsala, Sweden. \\ E-mail: fb.nieri@gmail.com; maxim.zabzine@physics.uu.se
}

Received: 15 August 2016/ Accepted: 9 February 2017

Published online: 11 April 2017 - (C) The Author(s) 2017. This article is an open access publication

\begin{abstract}
We study partition functions of $3 \mathrm{~d} \mathcal{N}=2 \mathrm{U}(N)$ gauge theories on compact manifolds which are $S^{1}$ fibrations over $S^{2}$. We show that the partition functions are free field correlators of vertex operators and screening charges of the $q$-Virasoro modular double, which we define. The inclusion of supersymmetric Wilson loops in arbitrary representations allows us to show that the generating functions of Wilson loop vacuum expectation values satisfy two $\operatorname{SL}(2, \mathbb{Z})$-related commuting sets of $q$-Virasoro constraints. We generalize our construction to $3 \mathrm{~d} \mathcal{N}=2$ unitary quiver gauge theories and as an example we give the free boson realization of the $\mathrm{ABJ}(\mathrm{M})$ model.
\end{abstract}

\section{Contents}

1. Introduction . . . . . . . . . . . . . . . . . . . 1060

2. Matrix Models, Free Fields and 3d Gauge Theories . . . . . . . . . . . . . 1064

2.1 Virasoro matrix model . . . . . . . . . . . . . . . . . . . . 1064

$2.2 q$-Virasoro matrix model . . . . . . . . . . . . . . . . . 1066

2.3 Gauge theory on $D^{2} \times S^{1} \ldots \ldots \ldots$. . . . . . . . . . . . . . . . . . . . . . . . . . . . . . . . .

3. Gauge Theory on $S_{b}^{3} \ldots \ldots \ldots \ldots \ldots$. . . . . . . . . . . . . . . . . . . . . 1071

3.1 Generating function . . . . . . . . . . . . . . . . . 1072

3.2 Free boson realization . . . . . . . . . . . . . . . . . . . . 1074

3.3 Round $S^{3}: \mathrm{W}_{1, t}$ and Virasoro limits . . . . . . . . . . . . . 1076

4. Other Compact Backgrounds . . . . . . . . . . . . . . . . . . . . . . . . 1079

$4.1 L(r, 1) \ldots \ldots \ldots \ldots$. . . . . . . . . . . . . . . . 1079

$4.2 S^{2} \times S^{1}$ (index) . . . . . . . . . . . . . . . . 1080

$4.3 S^{2} \times S^{1}$ (twisted index) . . . . . . . . . . . . . . . 1081

5. Inclusion of Chern-Simons Terms . . . . . . . . . . . . . . . . . . . 1084

5.1 Decoupling hyper multiplets . . . . . . . . . . . . . . . . 1085

5.2 Pure Chern-Simons and torus knots . . . . . . . . . . . . . . . . . . 1087

5.3 Refined Chern-Simons . . . . . . . . . . . . . . . . . . . . 1087 
6. Generalization to Quiver Gauge Theories . . . . . . . . . . . . . . . . . . 1088

$6.1 \mathrm{ABJ}(\mathrm{M})$ theory . . . . . . . . . . . . . . . . . . . . 1091

7. Summary, Comments and Outlook . . . . . . . . . . . . . . . . 1094

A. Special Functions . . . . . . . . . . . . . . . . . . . . . . 1096

References . . . . . . . . . . . . . . . . . . . . 1097

\section{Introduction}

Quantum field theories in three dimensions have played a relevant role in theoretical physics and many branches of mathematics since Witten's seminal work on Wilson loops in Chern-Simons theory and Jones polynomials [1]. Chern-Simons theory and its refined [2-4] or supersymmetric extensions feature prominently in topological string theory [57], in the study of the low energy physics of string/M-theory through Hanany-Witten brane constructions [8] or the celebrated $\mathrm{ABJ}(\mathrm{M})$ model for the effective theory of $\mathrm{M} 2$ branes $[9,10]$. The application of field theory methods for studying $3 \mathrm{~d}$ manifolds and knot theory has recently produced many new results and connections between the two fields, culminated in the discovery of the $3 \mathrm{~d}-3 \mathrm{~d}$ correspondence [11-14] (see also the review [15]) relating $3 \mathrm{~d} \mathcal{N}=2$ SCFTs arising from M5 branes compactified on a $3 \mathrm{~d}$ manifold to complex Chern-Simons on the latter [16]. More generally, the embedding of $3 \mathrm{~d} \mathcal{N}=2$ Chern-Simons-Yang-Mills theories in string/M-theory has provided many insights into their physics, including 3d Seiberg-like dualities [17-22] and mirror symmetry [23-25]. However, a better understanding of the rich dynamics and web of dualities of these field theories is desirable, perhaps exploiting some large symmetry hidden in this class of theories: this is the topic of this work.

In this paper we focus on a wide class of $3 \mathrm{~d} \mathcal{N}=2$ Yang-Mills-Chern-Simons (YMCS) unitary quiver gauge theories. A simple yet instrumental example for our analysis is the $\mathrm{U}(N)$ theory coupled to 1 adjoint chiral multiplet, which has a distinguished role also within the $3 \mathrm{~d}-3 \mathrm{~d}$ correspondence $[14,26,27]$. These theories can conveniently be studied on compact backgrounds [28-33] such as the squashed $S_{b}^{3}$, lens spaces $L(r, 1)$ and $S^{2} \times S^{1}$. The application of supersymmetric localization [34] to this class of theories [35-47] has been a powerful tool for studying non-perturbative gauge dynamics over the past few years. In fact, one of the main outcomes of the localization method is that expectation values of supersymmetric observables can be exactly computed by reducing path integrals to finite dimensional matrix models (Coulomb branch localization), which can then be analyzed from different angles. Our goal is to use these results to show that there is a universal algebraic structure underlying the supersymmetric sector of these theories in any such background, which we call the $W_{q, t}$ modular double, or $q$-Virasoro modular double for the single node quiver.

In order to explain our results it is enough to consider the reference example given by the $\mathrm{U}(N)$ theory coupled to 1 adjoint chiral multiplet and possibly (anti-)fundamental chiral multiplets, in which case the Coulomb branch partition function of the theory can be schematically written as

$$
Z=\sum_{\underline{\ell} \in \mathbb{F}^{N}} \int \mathrm{d}^{N} \underline{x} \Delta(\underline{x}, \underline{\ell}) \mathrm{e}^{\sum_{j} V\left(x_{j}, \ell_{j}\right)},
$$

where the continuous and discrete variables $\{\underline{x}, \underline{\ell}\}=\left\{x_{j}, \ell_{j}, j=1, \ldots, N\right\}$ parametrize the localization locus, $\Delta(\underline{x}, \underline{\ell})$ is the 1-loop contribution of the vector and adjoint multiplets, $\exp \left(\sum_{j} V\left(x_{j}, \ell_{j}\right)\right)$ is the 1-loop contribution of (anti-)fundamental matter and 
classical CS action, while the sum is over the different topological sectors: $\mathbb{F}=\left(\emptyset, \mathbb{Z}_{r}, \mathbb{Z}\right)$ for $S_{b}^{3}, L(r, 1)$ and $S^{2} \times S^{1}$ respectively. One of our main results is that 3d compact space partition functions are free boson correlators of vertex operators $(\mathcal{V}(z))$ and integrated screening currents $(\mathcal{S}(x))$ of a modular double version of the $q$-Virasoro algebra [48] which we define, namely

$$
Z=\int \mathrm{d}^{N} \underline{x}\left\langle\prod_{f} \mathcal{V}_{f}\left(z_{f}\right) \prod_{j=1}^{N} \mathcal{S}\left(x_{j}\right)\right\rangle,
$$

leading to a dual 2d CFT-like description in the spirit of the AGT correspondence [49$51]$ and similar to the proposal of $[52,53]$ for the SQED. The origin of the $q$-deformation has been proposed to lie in the little string deformation of the $6 \mathrm{~d}(2,0)$ theory [54].

The central object of our construction is the modular double screening current $\mathcal{S}(x)$. We define it to be the operator which commutes, up to total differences, with two commuting copies $(i=1,2)$ of the $q$-Virasoro generators $\left\{\mathrm{T}_{n, i}, n \in \mathbb{Z}\right\}_{i=1,2}$ whose $q$-deformation parameters are related by $\operatorname{SL}(2, \mathbb{Z})$ transformations, namely

$$
\begin{aligned}
{\left[\mathrm{T}_{n, 1}, \mathrm{~T}_{m, 2}\right] } & =0, \quad\left[\mathrm{~T}_{n, i}, \mathcal{S}(x)\right]=\text { total difference, } \\
q_{1} & =\mathrm{e}^{2 \pi \mathrm{i} \epsilon}, \quad q_{2}=\mathrm{e}^{-2 \pi \mathrm{i} g \cdot \epsilon},
\end{aligned}
$$

where $g \cdot$ is the standard $g \in \operatorname{SL}(2, \mathbb{Z})$ action on the modular parameter $\epsilon$. In order to avoid possible confusion, throughout this paper the index $i=1,2$ will be exclusively used for distinguishing the two copies and nothing else. The $q_{i}$ are related to geometric moduli of the gauge theory background (squashing or fibration parameters); in fact

$$
g \cdot \epsilon=\frac{\epsilon}{1-r \epsilon}
$$

for the lens space $L(r, 1)$, whereas $r=1,0$ for the particular cases $S_{b}^{3}$ and $S^{2} \times S^{1}$ respectively. The fact that the $q$-deformation parameters are related by $\operatorname{SL}(2, \mathbb{Z})$ is crucial: in this case we can give $\mathcal{S}(x)$ in terms of the screening currents $\mathrm{S}(w)_{i}$ of the individual $q$-Virasoro copies according to

$$
\mathcal{S}(x)=\sum_{\ell \in \mathbb{F}} w(x, \ell)_{1} w(x, \ell)_{2} \mathrm{~S}(w(x, \ell))_{1} \otimes \mathbf{S}(w(x, \ell))_{2},
$$

where the dependence of the summands on the gauge theory continuous and discrete variables is through the "holomorphic" coordinate $w(x, \ell)_{1}$ and its "conjugate" in the sense of the $\operatorname{SL}(2, \mathbb{Z})$ pairing, which acts also on the position in a certain way

$$
w(x, \ell)_{2}=g \cdot w(x, \ell)_{1} .
$$

From the gauge theory viewpoint, $w(x, \ell)_{i}$ are supersymmetric Wilson lines $\left(\boldsymbol{w}_{i}\right)$ at the North $(i=1)$ and South $(i=2)$ poles of the $S^{2}$ base

$$
\boldsymbol{w}_{i}=\operatorname{Pexp}\left(\mathrm{i} \oint_{\mathcal{C}_{i}}\left(A-\mathrm{i} \sigma\left|\dot{\mathcal{C}}_{i}\right| \mathrm{d} s\right)\right),
$$

evaluated at the localization locus and projected on a $\mathrm{U}(N)$ fundamental weight $\rho$

$$
w(x, \ell)_{1}=\rho\left(\boldsymbol{w}(\underline{x}, \underline{\ell})_{1}\right), \quad w(x, \ell)_{2}=\rho\left(\boldsymbol{w}(\underline{x}, \underline{\ell})_{2}\right),
$$


whose expressions depend on the specific background. Here $A$ denotes the gauge connection, $\sigma$ the vector multiplet scalar and $\mathcal{C}_{i}$ a supersymmetry preserving cycle.

The partition function is not the most general observable one can consider. Following what we have just mentioned, an important class of observables that can be computed through localization is given by supersymmetric Wilson loops. The evaluation of Wilson loop vacuum expectation values (v.e.v.) at the North or South poles of the $S^{2}$ base of the geometries we are considering amounts to insert

$$
\operatorname{Tr}_{\mathcal{R}_{i}}\left(\boldsymbol{w}(\underline{x}, \underline{\ell})_{i}\right)
$$

into the Coulomb branch partition function, where $\mathcal{R}_{i}$ is a representation of the $\mathrm{U}(N)$ gauge group. Using the standard character decomposition, we can package Wilson loop v.e.v.'s in arbitrary representations into the generating function

$$
Z\left(\underline{\tau}_{1}, \underline{\tau}_{2}\right)=\sum_{\mathcal{R}_{1}, \mathcal{R}_{2}} \sum_{\underline{\ell} \in \mathbb{F}^{N}} \int \mathrm{d}^{N} \underline{x} \Delta(\underline{x}, \underline{\ell}) \mathrm{e}^{\sum_{j} V\left(x_{j}, \ell_{j}\right)} \prod_{i=1,2} \operatorname{Tr}_{\mathcal{R}_{i}}\left(\boldsymbol{v}\left(\underline{\tau}_{i}\right)\right) \operatorname{Tr}_{\mathcal{R}_{i}}\left(\boldsymbol{w}(\underline{x}, \underline{\ell})_{i}\right),
$$

where the additional insertions $\operatorname{Tr}_{\mathcal{R}_{i}}\left(\boldsymbol{v}\left(\underline{\tau}_{i}\right)\right)$ can be thought of as background Wilson loops. The generating function is the natural object to consider from a matrix model perspective, and we can give it a $q$-Virasoro interpretation as well: for YM theories it can be identified with the highest weight state

$$
Z\left(\underline{\tau}_{1}, \underline{\tau}_{2}\right) \simeq \int \mathrm{d}^{N} \underline{x} \prod_{j=1}^{N} \mathcal{S}\left(x_{j}\right)|\alpha\rangle
$$

In this language, (anti-)fundamental matter can be coupled to the gauge theory by shifting the "time" variables $\underline{\tau}_{i}$ (isomorphic to the creation operators in the free boson representation of $q$-Virasoro), or equivalently by acting on the state with additional vertex operators. Inclusion of CS terms can be dealt with similarly. Remarkably, our identification implies the existence of two $\operatorname{SL}(2, \mathbb{Z})$-related commuting sets of $q$-Virasoro constraints (or Ward identities) satisfied by the YM generating function

$$
T_{n}\left(\underline{\tau}_{i}\right) Z\left(\underline{\tau}_{1}, \underline{\tau}_{2}\right)=0, \quad n>0,
$$

where $T_{n}\left(\underline{\tau}_{i}\right) \simeq \mathrm{T}_{n, i}$ are differential operators in $\underline{\tau}_{i}$, which express the highest weight condition of the YM generating function. A similar description holds when including CS terms. This observation opens up the possibility of characterizing compact space generating functions as solutions of two infinite sets of PDEs. Similar considerations have been put forward in $[12-14,55,56]$, where it is shown that the algebra of line operators and their action on $3 \mathrm{~d}$ partition functions gives rise to recurrence relations quantizing classical spectral curves or knot polynomials, and in [57-60], where the relation of line operators with difference operators/quantum Hamiltonians of integrable systems is discussed.

Our results fit nicely with the observed factorization properties of $3 \mathrm{~d}$ compact space partition functions [13,58,61-67]. All the manifolds we are interested in admit indeed a decomposition into a pair of solid tori $\left(D^{2} \times S^{1}\right)_{i=1,2}$, where the boundary homeomorphism is implemented by the $g \in \operatorname{SL}(2, \mathbb{Z})$ element acting on one boundary torus with modulus $\epsilon$. Gauge theory partition functions on the diverse compact spaces can be recovered by $\operatorname{SL}(2, \mathbb{Z})$ gluings of partition functions on the solid torus $D^{2} \times S^{1}$. 
Supersymmetric partition functions on such elementary background are known as $3 \mathrm{~d}$ holomorphic blocks [13]

$$
\mathcal{B}_{c}^{3 \mathrm{~d}}=\oint_{c} \mathrm{~d}^{N} \underline{w} \Upsilon^{3 \mathrm{~d}}(\underline{w}),
$$

and for $\mathrm{U}(N)$ YM theories they have been shown [68] (see also the review [69]) to be captured by free boson correlators of $q$-Virasoro screening currents and vertex operators $(\mathrm{H}(z))$

$$
\mathcal{B}_{c}^{3 \mathrm{~d}}=\oint_{c} \mathrm{~d}^{N} \underline{w}\left\langle\prod_{f} \mathrm{H}_{f}\left(z_{f}\right) \prod_{j=1}^{N} \mathrm{~S}\left(w_{j}\right)\right\rangle .
$$

Our construction then reveals the algebraic structure behind the observed non-trivial decomposition [62,67]

$$
Z=\sum_{\underline{\ell} \in \mathbb{F}^{N}} \int \mathrm{d}^{N} \underline{x} \Upsilon^{3 \mathrm{~d}}(\underline{w}(\underline{x}, \underline{\ell}))_{1} \Upsilon^{3 \mathrm{~d}}(\underline{w}(\underline{x}, \underline{\ell}))_{2}
$$

of compact space partition functions. Moreover, in many cases it has been shown that $Z$ can be completely factorized as

$$
Z=\sum_{\{c\}}\left(\mathcal{B}_{c}^{3 \mathrm{~d}}\right)_{1}\left(\mathcal{B}_{c}^{3 \mathrm{~d}}\right)_{2},
$$

where the sum is over the supersymmetric massive vacua of the effective $2 \mathrm{~d}$ theory on the cigar or flat connections in complex CS through the $3 \mathrm{~d}-3 \mathrm{~d}$ correspondence. Our general results explain this property from the existence of two commuting sets of $q$-Virasoro constraints satisfied by the generating functions.

Finally, our results can also be read in the context of the BPS/CFT correspondence and $5 \mathrm{~d}$ AGT. Supersymmetric $5 \mathrm{~d}$ unitary quiver gauge theories in the $\Omega$-background have an interesting class of observables known as $q q$-characters, which have been recently constructed in [70] (building on previous works [71,72]). In particular, it is shown in [73] that the $q q$-characters generate quiver $\mathrm{W}_{q, t}$ symmetry algebras and Ward identities for $5 \mathrm{~d}$ (extended) Nekrasov partition functions $[74,75]$. When $5 \mathrm{~d}$ gauge theories can be engineered by M-theory compactifications on toric Calabi-Yau 3-folds [76,77] or type IIB $(p, q)$-webs [78,79], one can also use the refined topological vertex formalism [8082] to conveniently compute the $5 \mathrm{~d}$ Nekrasov partition functions. Using this approach it has been recently realized $[83,84]$ that 5 d gauge theories supported on $(p, q)$-webs form a representation of the Ding-Iohara-Miki algebra $[85,86]$, which is the building block for constructing $\mathrm{W}_{q, t}$ algebras (at least in the $A_{n}$ case) as much as the strip geometry [87] is the building block for constructing toric webs. In any case, the free boson representation of the relevant symmetry algebra yields a matrix model description of the $5 \mathrm{~d}$ Nekrasov partition function [88-95], which at isolated points on the Coulomb branch describes a $3 d$ vortex theory $[54,68,69,96]$. We thus expect that our construction describes the compact space version, or the non-perturbative completion in the sense of [97], of this chain of dualities between gauge/string theory and quantum algebras.

The rest of this paper is organized as follows. In Sect. 2, we review some basics in the theory of conformal matrix models and Virasoro constraints, and the analogous constructions for the $q$-deformed case. We also review the $q$-Virasoro description of $3 \mathrm{~d}$ holomorphic blocks of $\mathrm{U}(N)$ YM theories, and propose the $q$-Virasoro interpretation of the Wilson loop generating function. In Sect. 3, we discuss in detail $\mathrm{U}(N)$ YM theories 
on the squashed $S_{b}^{3}$, focusing on the Coulomb branch partition function and Wilson loop generating function. We then discuss how these objects can be mapped to correlators or highest weight states of the $q$-Virasoro modular double and we find two SL(2, $\mathbb{Z})$ related commuting sets of $q$-Virasoro constraints annihilating the generating function. We also comment on a few interesting limits of the gauge theory and the associated constraints, such as the round $S^{3}$ or special values of the adjoint mass. In Sect. 4, we extend our analysis to the lens space partition function, the index and twisted index. In Sect. 5, we discuss how CS terms can be described in the $q$-Virasoro side, leading to "dressed" correlators and modified $q$-Virasoro constraints. In Sect. 6, we consider the generalization to quiver gauge theories, with special focus on the $\mathrm{ABJ}(\mathrm{M})$ theory, and the relation to quiver $\mathrm{W}_{q, t}$ algebras. In Sect. 7, we summarize our results and comment on open questions and interesting directions for future work, such as the possible $4 \mathrm{~d} / \mathrm{elliptic}$ lift of our construction and the relation to $5 \mathrm{~d}$ theories.

\section{Matrix Models, Free Fields and 3d Gauge Theories}

In this section we summarize basic facts about the $\beta$-ensemble and its free boson Virasoro construction, while for a detailed review we refer to $[98,99]$. This elementary discussion will allow us to introduce the main tools which also apply to the $q$-deformed $\beta$-ensemble and $q$-Virasoro algebra, for details we refer to $[88,100]$. We then recall the $q$-Virasoro interpretation of $3 \mathrm{~d}$ partition functions on $D^{2} \times S^{1}$ given by [68], and we extend the duality by mapping the Wilson loop generating function to the $q$-deformed $\beta$-ensemble.

\subsection{Virasoro matrix model. Let us consider the matrix model}

$$
Z(\underline{\tau})=\mathcal{N}_{0} \int \mathrm{d}^{N} \underline{w} \Delta_{\beta}(\underline{w}) \mathrm{e}^{\sqrt{\beta} \sum_{j} V\left(w_{j} \mid \underline{\tau}\right)}, \quad V(w \mid \underline{\tau})=\sum_{n>0} \tau_{n} w^{n},
$$

where $\Delta_{\beta}(\underline{w})$ is the integration measure describing the interactions between the eigenvalues $\underline{w}, V(w \mid \underline{\tau})$ is the potential whose shape is described by the time parameters $\underline{\tau}, \mathcal{N}_{0}$ is a normalization parametrized by $\tau_{0}$ and $\beta \in \mathbb{C}$. We refer to such a partition function as a Virasoro matrix model if it satisfies the Virasoro constraints

$$
L_{n}(\underline{\tau}) Z(\underline{\tau})=0, \quad n \in \mathbb{Z}_{>0},
$$

where $L_{n}(\underline{\tau})$ are differential operators in the time variables satisfying the positive mode subalgebra of the full Virasoro algebra

$$
\left[L_{n}, L_{m}\right]=(n-m) L_{n+m}+\frac{c}{12} n\left(n^{2}-1\right) \delta_{n+m, 0}, \quad n, m \in \mathbb{Z} .
$$

The fact that the matrix model partition function depends on infinitely many parameters and that it is subject to infinitely many constraints forming a closed algebra, is a strong indication that the matrix model can be defined as the (unique) solution to the Virasoro constraint equations (with suitable boundary conditions).

A simple method to built the matrix model satisfying Virasoro constraints is exploiting the free boson realization of the Virasoro algebra. Let us consider the Heisenberg algebra (we display non-trivial relations only)

$$
\left[\mathrm{a}_{n}, \mathrm{a}_{m}\right]=2 n \delta_{n+m, 0}, \quad[\mathrm{P}, \mathrm{Q}]=2, \quad n, m \in \mathbb{Z} \backslash\{0\},
$$


and the Fock module $\mathcal{F}_{\alpha}$ over the charged vacuum $|\alpha\rangle$ spanned by the states

$$
\mathcal{F}_{\alpha}=\left\{\prod_{n=1}^{|\mu|} \mathrm{a}_{-\mu_{n}}|\alpha\rangle\right\}, \quad \prod_{n=1}^{|\mu|} \mathrm{a}_{\mu_{n}}|\alpha\rangle=0, \quad|\alpha\rangle=\mathrm{e}^{\frac{\alpha}{2} \mathrm{Q}}|0\rangle, \quad \mathrm{P}|\alpha\rangle=\alpha|\alpha\rangle,
$$

for any partition $\mu$ of length $|\mu|$ and given momentum $\alpha \in \mathbb{C}$. The operators

$$
\begin{aligned}
& \mathrm{L}_{n}=\frac{1}{4} \sum_{k \neq 0, n}: \mathrm{a}_{n-k} \mathrm{a}_{k}:+\frac{1}{2} \mathrm{a}_{n} \mathrm{P}-\frac{1}{2} Q_{\beta}(n+1) \mathrm{a}_{n}, \quad n \neq 0, \\
& \mathrm{~L}_{0}=\frac{1}{2} \sum_{k>0} \mathrm{a}_{-k} \mathrm{a}_{k}+\frac{\mathrm{P}^{2}}{4}-\frac{1}{2} \mathrm{P} Q_{\beta}, \quad Q_{\beta}=\sqrt{\beta}-\frac{1}{\sqrt{\beta}},
\end{aligned}
$$

where : : denotes normal ordering (i.e. positive modes to the right of negative modes and $\mathrm{P}$ to the right of $\mathrm{Q}$ ), close the Virasoro algebra (2.3) with central charge $c=1-6 Q_{\beta}^{2}$. Using the algebra representation

$$
\mathrm{a}_{-n} \simeq n \tau_{n}, \quad \mathrm{a}_{n} \simeq 2 \frac{\partial}{\partial \tau_{n}}, \quad \mathrm{Q} \simeq \tau_{0}, \quad \mathrm{P} \simeq 2 \frac{\partial}{\partial \tau_{0}}, \quad|\alpha\rangle=\mathrm{e}^{\frac{\alpha}{2} \mathrm{Q}}|0\rangle \simeq \mathrm{e}^{\tau_{0} \frac{\alpha}{2}} \cdot 1,
$$

we get the differential representation

$$
\mathrm{L}_{n} \simeq L_{n}(\underline{\tau})=\sum_{k \geq 0} k \tau_{k} \frac{\partial}{\partial \tau_{n+k}}+\sum_{k=0}^{n} \frac{\partial^{2}}{\partial \tau_{n-k} \partial \tau_{k}}-Q_{\beta}(n+1) \frac{\partial}{\partial \tau_{n}}, \quad n>0 .
$$

Then the original problem (2.2) can be solved by finding a free boson operator $\mathrm{S}(w)$ whose commutator with the Virasoro generators is a total derivative, namely

$$
\left[\mathrm{L}_{n}, \mathrm{~S}(w)\right]=\frac{\mathrm{d}}{\mathrm{d} w} \mathrm{O}(w)
$$

for some (n-dependent) operator $\mathrm{O}(w)$. In fact, by defining ${ }^{1}$

$$
\mathbf{Z}=\mathrm{J}^{N}, \quad \mathbf{J}=\int \mathrm{d} w \mathrm{~S}(w),
$$

through the representation (2.7) we immediately get ${ }^{2}$

$$
\mathrm{Z}|\alpha\rangle \simeq Z(\underline{\tau}), \quad \mathrm{L}_{n} \mathrm{Z}|\alpha\rangle \simeq L_{n}(\underline{\tau}) Z(\underline{\tau})=0, \quad n>0,
$$

where the last equality follows from the screening charge conservation $\left[L_{n}, J\right]=0$ and the highest weight condition $L_{n>0}|\alpha\rangle=0 .{ }^{3}$ Notice that by packaging the Virasoro

\footnotetext{
1 A suitable choice of integration contour is to be understood.

2 This identification is usually achieved by an explicit projection onto the coherent state $\left\langle\alpha_{\infty}\right| \mathrm{G}(\underline{\tau}), \mathrm{G}(\underline{\tau})=$ $\exp \left(\frac{1}{2} \sum_{n>0} \tau_{n} \mathrm{a}_{n}\right)$, with $\alpha_{\infty}=\alpha+2 \sqrt{\beta} N$ and $\left\langle\alpha_{\infty}\right|$ the dual charged Fock vacuum $\left(\left\langle\alpha_{a} \mid \alpha_{b}\right\rangle=\delta_{a b}\right)$. This projection is equivalent to the representation (2.7): $2 \frac{\partial}{\partial \tau_{n}}\left\langle\alpha_{\infty}\right| \mathrm{G}(\underline{\tau})=\left\langle\alpha_{\infty}\right| \mathrm{G}(\underline{\tau}) \mathrm{a}_{n}, n \tau_{n}\left\langle\alpha_{\infty}\right| \mathrm{G}(\underline{\tau})=$ $\left\langle\alpha_{\infty}\right| \mathrm{G}(\underline{\tau}) \mathrm{a}_{-n}, \alpha_{\infty}\left\langle\alpha_{\infty}\right| \mathrm{G}(\underline{\tau})=\left\langle\alpha_{\infty}\right| \mathrm{G}(\underline{\tau}) \mathrm{P}=2 \frac{\partial}{\partial \tau_{0}}\left\langle\alpha_{\infty}\right| \mathrm{G}(\underline{\tau})$.

${ }^{3}$ For $\alpha=0$ there are the additional constraints $n=-1,0$ due to $\mathfrak{s l}_{2}$ invariance of the vacuum $|0\rangle$.
} 
generators into the current (stress tensor) $\mathrm{L}(z)=\sum_{n \in \mathbb{Z}} \mathrm{L}_{n} z^{-n-2}$, the constraints (2.2) are equivalent to the regularity condition (Ward identities)

$$
z^{2} L(z \mid \underline{\tau}) Z(\underline{\tau})=\operatorname{Pol}(z)
$$

for a certain ( $\underline{\tau}$-dependent) polynomial $\operatorname{Pol}(z)$.

The central object of the free boson construction is the screening current $\mathrm{S}(w)$ defined by (2.9), and its free boson representation is given by

$$
\mathrm{S}(w)=: \mathrm{e}^{-\sqrt{\beta} \sum_{n \neq 0} \frac{w^{-n}}{n} \mathrm{a}_{n}}: \mathrm{e}^{\sqrt{\beta} \mathrm{Q}} w^{\sqrt{\beta} \mathrm{P}} .
$$

This representation allows us to write down the matrix model explicitly, in fact

$$
\prod_{j=1}^{N} \mathrm{~S}\left(w_{j}\right)=: \prod_{j=1}^{N} \mathrm{~S}\left(w_{j}\right): \Delta_{\beta}(\underline{w}), \quad \Delta_{\beta}(\underline{w})=\prod_{k<j}\left(w_{k}-w_{j}\right)^{2 \beta},
$$

and hence

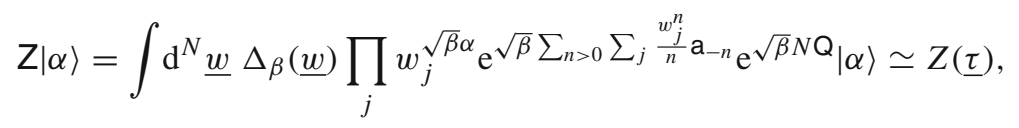

with $V(w \mid \underline{\tau})=\alpha \ln w+\sum_{n>0} \tau_{n} w^{n}, \mathcal{N}_{0}=\exp \sqrt{\beta} \tau_{0}\left(N+\frac{\alpha}{2 \sqrt{\beta}}\right)$, where we used (2.7).

We conclude this brief review of Virasoro matrix models with three remarks. Firstly, this $2 \mathrm{~d}$ CFT construction can be easily generalized to other (quantum) algebras provided that the free boson representation of generators and screening currents is known. For instance, this is the case with $\mathrm{W}$ algebras and their $q$-deformation, which will be in fact the main focus of this paper. Secondly, different looking matrix models may actually be related by a simple redefinition of the time variables. Thirdly, the matrix model can be enriched through the inclusion of vertex operators in the free boson correlator. In $2 \mathrm{~d}$ CFTs there is a distinguished set of operators called primaries, which in the free boson representation are given by

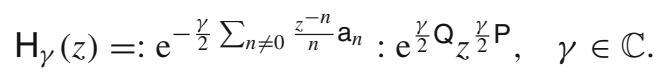

Their OPE with the screening current is

$$
\mathrm{H}_{\gamma}(z) \mathrm{S}(w)=: \mathrm{H}_{\gamma}(z) \mathrm{S}(w):\left(1-w z^{-1}\right)^{\sqrt{\beta} \gamma} z^{\sqrt{\beta} \gamma},
$$

whose effect is simply to add a constant background to the time variables

$$
\tau_{n} \rightarrow \tau_{n}-\gamma \frac{z^{-n}}{n}
$$

and to change the normalization by a constant multiplicative factor. For this reason we will be mainly interested in theories without vertex operators.

2.2. $q$-Virasoro matrix model. The $q$-Virasoro algebra is the associative algebra generated by $\left\{T_{n}, n \in \mathbb{Z}\right\}$ satisfying the relation [48] 


$$
\begin{aligned}
f & \left(\frac{w}{z}\right) T(z) T(w)-f\left(\frac{z}{w}\right) T(w) T(z) \\
& =-\frac{(1-q)\left(1-t^{-1}\right)}{(1-p)}\left(\delta\left(p \frac{w}{z}\right)-\delta\left(p^{-1} \frac{w}{z}\right)\right),
\end{aligned}
$$

where

$$
T(z)=\sum_{n \in \mathbb{Z}} T_{n} z^{-n}, \quad f(z)=\sum_{\ell \geq 0} f_{\ell} z^{\ell}=\mathrm{e}^{\sum_{n>0} \frac{\left(1-q^{n}\right)\left(1-t^{-n}\right)}{n\left(1+p^{n}\right)} z^{n}}, \quad \delta(z)=\sum_{n \in \mathbb{Z}} z^{n},
$$

and $q, t, p \in \mathbb{C}$ with $p=q t^{-1}$. This algebra provides a 1-parameter deformation of the Virasoro algebra. In fact, upon setting $t=q^{\beta}, q=\mathrm{e}^{\hbar}$, we have the small $\hbar \in \mathbb{R}$ expansion

$$
T_{n}=2 \delta_{n, 0}+\hbar^{2} \beta\left(L_{n}+\frac{Q_{\beta}^{2}}{4} \delta_{n, 0}\right)+O\left(\hbar^{4}\right),
$$

where the operators $L_{n}$ close the Virasoro algebra (2.3) with central charge $c=1-6 Q_{\beta}^{2}$.

The Heisenberg algebra (we display non-trivial relations only)

$\left[\mathrm{a}_{n}, \mathrm{a}_{m}\right]=\frac{1}{n}\left(q^{\frac{n}{2}}-q^{-\frac{n}{2}}\right)\left(t^{\frac{n}{2}}-t^{-\frac{n}{2}}\right)\left(p^{\frac{n}{2}}+p^{-\frac{n}{2}}\right) \delta_{n+m, 0}, \quad[\mathrm{P}, \mathrm{Q}]=2, \quad n, m \in \mathbb{Z} \backslash\{0\}$,

gives a free boson representation of the $q$-Virasoro algebra according to

$$
\mathrm{T}(z)=\sum_{n \in \mathbb{Z}} \mathrm{T}_{n} z^{-n}=\sum_{\sigma= \pm 1} \Lambda_{\sigma}(z), \quad \Lambda_{\sigma}(z)=: \mathrm{e}^{\sigma \sum_{n \neq 0} \frac{z^{-n}}{\left(1+p^{-\sigma n}\right)} \mathrm{a}_{n}}: q^{\sigma \frac{\sqrt{\beta}}{2} \mathrm{P}} p^{\frac{\sigma}{2}}
$$

where $\beta=\ln t / \ln q$. The $q$-Virasoro screening current is given by ${ }^{4}$

$$
\mathrm{S}(w)=: \mathrm{e}^{-\sum_{n \neq 0} \frac{w^{-n}}{q^{n / 2}-q^{-n / 2}} \mathrm{a}_{n}}: \mathrm{e}^{\sqrt{\beta} \mathrm{Q}} w^{\sqrt{\beta} \mathrm{P}} .
$$

One can indeed verify that the defining relation

$$
\left[\mathrm{T}_{n}, \mathrm{~S}(w)\right]=\frac{\mathrm{O}(q w)-\mathrm{O}(w)}{w}
$$

holds true for a certain ( $n$-dependent) operator $\mathrm{O}(w)$, implying the conservation of the screening charge for a suitable contour, $\left[\mathrm{T}_{n}, \oint \mathrm{d} w \mathrm{~S}(w)\right]=0$.

The $q$-Virasoro matrix model can now be constructed by exploiting the strategy outlined in the previous subsection. The product of several $q$-Virasoro screening currents yields

$$
\prod_{j=1}^{N} \mathrm{~S}\left(w_{j}\right)=: \prod_{j=1}^{N} \mathrm{~S}\left(w_{j}\right): \Delta_{\beta}(\underline{w} ; q) c_{\beta}(\underline{w}, 1 ; q) \prod_{j} w_{j}^{\beta(N-1)},
$$

with

$$
\Delta_{\beta}(\underline{w} ; q)=\prod_{k \neq j} \frac{\left(w_{k} w_{j}^{-1} ; q\right)_{\infty}}{\left(t w_{k} w_{j}^{-1} ; q\right)_{\infty}}, \quad c_{\beta}(\underline{w}, m ; q)=\prod_{k<j}\left(w_{k} w_{j}^{-1}\right)^{\beta} \frac{\Theta\left(t m w_{k} w_{j}^{-1} ; q\right)}{\Theta\left(m w_{k} w_{j}^{-1} ; q\right)},
$$

\footnotetext{
4 There is another screening current with $q \rightarrow t^{-1}, \sqrt{\beta} \rightarrow-\frac{1}{\sqrt{\beta}}$, but we do not need it in this work.
} 
or

$$
\Delta_{\beta}(\underline{w} ; q) c_{\beta}(\underline{w} ; q)=\prod_{k<j}\left(w_{k} w_{j}^{-1}\right)^{\beta}\left(1-w_{j} w_{k}^{-1}\right) \frac{\left(q t^{-1} w_{j} w_{k}^{-1} ; q\right)_{\infty}}{\left(t w_{j} w_{k}^{-1} ; q\right)_{\infty}}
$$

where the $q$-Pochhammer symbol and $\Theta$ function are defined in (A.1) and (A.5) respectively. As before, we can now define the operator

$$
\mathbf{Z}=\mathrm{J}^{N}, \quad \mathrm{~J}=\oint \mathrm{d}^{N} w \mathrm{~S}(w)
$$

and consider the state

$$
\begin{aligned}
\mathrm{Z}|\alpha\rangle= & \oint \frac{\mathrm{d}^{N} \underline{w}}{2 \pi \underline{\mathrm{i}} \underline{w}} \Delta_{\beta}(\underline{w} ; q) c_{\beta}(\underline{w}, 1 ; q) \\
& \prod_{j} w_{j}^{\sqrt{\beta}\left(\alpha+\sqrt{\beta} N-Q_{\beta}\right)} \mathrm{e}^{\sum_{n>0} \frac{\sum_{j} w_{j}^{n}}{q^{n / 2}-q^{-n / 2}} \mathrm{a}_{-n}} \mathrm{e}^{\sqrt{\beta} N \mathrm{Q}}|\alpha\rangle .
\end{aligned}
$$

Finally, the algebra representation

$$
\begin{aligned}
\mathrm{a}_{-n} & \simeq\left(q^{\frac{n}{2}}-q^{-\frac{n}{2}}\right) \tau_{n}, \quad \mathrm{a}_{n} \simeq \frac{1}{n}\left(t^{\frac{n}{2}}-t^{-\frac{n}{2}}\right)\left(p^{\frac{n}{2}}+p^{-\frac{n}{2}}\right) \frac{\partial}{\partial \tau_{n}}, \quad n \in \mathbb{Z}_{>0}, \\
\sqrt{\beta} \mathrm{Q} & \simeq \tau_{0}, \quad \mathrm{P} \simeq \frac{2}{\sqrt{\beta}} \frac{\partial}{\partial \tau_{0}}, \quad|\alpha\rangle=\mathrm{e}^{\frac{\alpha}{2} \mathrm{Q}}|0\rangle \simeq \mathrm{e}^{\tau_{0} \frac{\alpha}{2 \sqrt{\beta}}} \cdot 1,
\end{aligned}
$$

yields the matrix model

$$
\begin{aligned}
Z|\alpha\rangle \simeq Z(\underline{\tau}) & =\mathcal{N}_{0} \oint \frac{\mathrm{d}^{N} \underline{w}}{2 \pi \underline{\mathrm{w}} \underline{w}} \Delta_{\beta}(\underline{w} ; q) c_{\beta}(\underline{w}, 1 ; q) \mathrm{e}^{\sum_{j} V\left(w_{j} \mid \underline{\tau}\right)}, \\
V(w \mid \underline{\tau}) & =\sqrt{\beta}\left(\alpha+\sqrt{\beta} N-Q_{\beta}\right) \ln w+\sum_{n>0} \tau_{n} w^{n}, \quad \mathcal{N}_{0}=\mathrm{e}^{\tau_{0}\left(N+\frac{\alpha}{2 \sqrt{\beta}}\right)} .
\end{aligned}
$$

Due to the conservation of the screening charge $\left[\mathrm{T}_{n}, \mathrm{~J}\right]=0$ and the highest weight condition $\mathrm{T}_{n>0}|\alpha\rangle=0$, the above partition function satisfies $q$-Virasoro constraints by construction [88]

$$
T(z \mid \underline{\tau}) Z(\underline{\tau})=\operatorname{Pol}(z) \Rightarrow T_{n}(\underline{\tau}) Z(\underline{\tau})=0, \quad n>0,
$$

where the operators $T_{n}(\underline{\tau})$ can be read from the modes $T_{n}$ using (2.31). Explicitly, we have

$$
\mathrm{T}_{n}=\left\{\begin{array}{ll}
\sum_{\sigma= \pm 1} q^{\sigma \frac{\sqrt{\beta}}{2} \mathrm{P}} p^{\frac{\sigma}{2}} \sum_{k \geq 0} \frac{B_{k}\left(\left\{A_{-k}^{(\sigma)}\right\}\right) B_{n+k}\left(\left\{A_{n+k}^{(\sigma)}\right\}\right)}{(n+k) ! k !}, & n \geq 0 \\
\sum_{\sigma= \pm 1} q^{\sigma \frac{\sqrt{\beta}}{2} \mathrm{P}} p^{\frac{\sigma}{2}} \sum_{k \geq 0} \frac{B_{k-n}\left(\left\{A_{n-k}^{(\sigma)}\right\}\right) B_{k}\left(\left\{A_{k}^{(\sigma)}\right\}\right)}{(k-n) ! k !}, & n<0
\end{array},\right.
$$

where we set

$$
A_{n}^{(\sigma)}=\sigma \frac{\mathrm{a}_{n}|n| !}{\left(1+p^{-\sigma n}\right)}, \quad B_{n}\left(\left\{A_{n}\right\}\right)=B_{n}\left(A_{1}, \ldots, A_{n}\right), \quad B_{0}=1,
$$


with $B_{n}\left(\left\{A_{n}\right\}\right)$ the complete Bell polynomial defined by $\exp \sum_{n>0} \frac{A_{n}}{n !} z^{n}=$ $\sum_{n \geq 0} \frac{B_{n}\left(\left\{A_{n}\right\}\right)}{n !} z^{n}$.

As we have remarked at the end of the previous subsection, we can enrich the matrix model with the inclusion of vertex operators. The $q$-deformation of the operator (2.16) which is usually employed is [88] (see also [101] for a recent discussion)

$$
\mathrm{H}_{\gamma}(z)=: \mathrm{e}^{-\sum_{n \neq 0} \frac{\left(t^{\gamma n / 2}-t^{-\gamma n / 2}\right) z^{-n}}{\left(q^{n / 2}-q^{-n / 2}\right)\left(t^{n / 2}-t^{-n / 2}\right)} \lambda_{n}}: \mathrm{e}^{\frac{\gamma}{2} \sqrt{\beta} \mathrm{Q}} z^{\frac{\gamma}{2} \sqrt{\beta} \mathrm{P}},
$$

where we have introduced the new basis

$$
\lambda_{n}=\mathrm{a}_{n}\left(p^{n / 2}+p^{-n / 2}\right)^{-1}
$$

of the Heisenberg algebra. The OPE with the $q$-Virasoro screening current is

$$
\mathrm{H}_{\gamma}(z) \mathrm{S}(w)=: \mathrm{H}_{\gamma}(z) \mathrm{S}(w): \frac{\left(q^{\frac{1}{2}} t^{-\frac{\gamma}{2}} w z^{-1} ; q\right)_{\infty}}{\left(q^{\frac{1}{2}} t^{\frac{\gamma}{2}} w z^{-1} ; q\right)_{\infty}} z^{\beta \gamma},
$$

and thus the inclusion of such a vertex operator simply amounts to add a constant background to the time variables

$$
\tau_{n} \rightarrow \tau_{n}-\frac{\left(q^{\frac{1}{2}} t^{-\frac{\gamma}{2}} z^{-1}\right)^{n}}{n\left(1-q^{n}\right)}+\frac{\left(q^{\frac{1}{2}} t^{\frac{\gamma}{2}} z^{-1}\right)^{n}}{n\left(1-q^{n}\right)},
$$

and to modify the normalization by a constant multiplicative factor. However, for our purposes it is also convenient to consider the "half" vertex operator

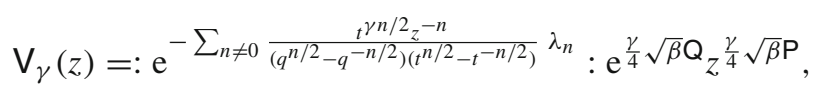

whose inclusion will shift the time variables by the last term in (2.39). With this definition, we also have

$$
\mathrm{H}_{\gamma}(z)=: \mathrm{V}_{\gamma}(z) \mathrm{V}_{-\gamma}(z)^{-1}:
$$

2.3. Gauge theory on $D^{2} \times S^{1}$. In this subsection we review a relevant application of the $q$-Virasoro theory for the study of $3 \mathrm{~d} \mathcal{N}=2$ gauge theories, and we also propose a gauge theory interpretation of the $q$-Virasoro matrix model (2.32), extending the results of $[68,69]$.

Partition functions of $3 \mathrm{~d} \mathcal{N}=2$ gauge theories compactified on $D^{2} \times S^{1}$ are computed by $3 \mathrm{~d}$ holomorphic block integrals introduced in [62] (see also [102] for derivation through localization)

$$
\mathcal{B}_{c}^{3 \mathrm{~d}}=\oint_{c} \frac{\mathrm{d}^{\mathrm{rk} G} \underline{w}}{2 \pi \mathrm{i} \underline{w}} \Upsilon^{3 \mathrm{~d}}(\underline{w}),
$$

where the integral kernel $\Upsilon^{3 \mathrm{~d}}(\underline{w})$ is determined by the specific theory with gauge group $G$ and the integration is over a basis of middle dimensional cycles $\{c=1, \ldots\}$ in $\left(\mathbb{C}^{\times}\right)^{\mathrm{rk} G}$. The vector multiplet contributes with the 1-loop factor ${ }^{5}$

$$
\Upsilon_{\operatorname{vec}}^{3 \mathrm{~d}}(\underline{w})=\prod_{\alpha \neq 0}\left(w_{\alpha} ; q\right)_{\infty},
$$

\footnotetext{
5 There can be anomalous terms in the 1-loop factors represented by quadratic polynomials. We will simply omit these factors because they vanish for the theories we are considering in this section.
} 
where $\alpha$ is a root of the gauge Lie algebra, while a chiral multiplet in a gauge representation $\mathcal{R}$ contributes with

$$
\Upsilon_{\mathrm{N}}^{3 \mathrm{~d}}(\underline{w}, m)=\prod_{\rho \in \mathcal{R}} \frac{1}{\left(w_{\rho} m ; q\right)_{\infty}}, \quad \text { or } \quad \Upsilon_{\mathrm{D}}^{3 \mathrm{~d}}(\underline{w}, m)=\prod_{\rho \in \mathcal{R}}\left(q w_{\rho}^{-1} m^{-1} ; q\right)_{\infty},
$$

where $\rho$ is a weight of $\mathcal{R}, m$ is a global $\mathrm{U}(1)$ fugacity and the index $\mathrm{N}$ or D refers to Neumann or Dirichlet boundary conditions. The $\epsilon$ parameter

$$
q=\mathrm{e}^{2 \pi \mathrm{i} \epsilon}
$$

can be interpreted as the disk equivariant parameter $\left(D^{2} \times S^{1} \simeq \mathbb{R}_{\epsilon}^{2} \times S^{1}\right)$ or as the modular parameter of the boundary torus $\left(\partial\left(D^{2} \times S^{1}\right) \simeq \mathbb{T}^{2}\right)$. Moreover, one can consider additional $2 \mathrm{~d}$ vector, Fermi or chiral multiplets on the boundary torus and contributing to the integral kernel respectively with [103-105]

$$
\prod_{\alpha \neq 0} \Theta\left(w_{\alpha} ; q\right), \quad \prod_{\rho \in \mathcal{R}} \Theta\left(w_{\rho} m ; q\right)^{ \pm 1} .
$$

These contributions are important to introduce the correct CS units and ensure local and large gauge invariance $[56,62,102]$.

As a concrete example we can consider the $\mathrm{U}(N)$ YM theory coupled to 1 adjoint chiral multiplet and additional $N_{\mathrm{f}}$ fundamental and anti-fundamental chiral multiplets, in which case the $3 \mathrm{~d}$ holomorphic block integral can be written as

$$
\mathcal{B}_{c}^{3 \mathrm{~d}}=\oint_{c} \frac{\mathrm{d}^{N} \underline{w}}{2 \pi \underline{\mathrm{i}} \underline{w}} \prod_{k \neq j=1}^{N} \frac{\left(w_{k} w_{j}^{-1} ; q\right)_{\infty}}{\left(m_{\mathrm{a}} w_{k} w_{j}^{-1} ; q\right)_{\infty}} \prod_{j=1}^{N} w_{j}^{\kappa_{1}} \prod_{f=1}^{N_{\mathrm{f}}} \frac{\left(q w_{j} \bar{m}_{f} ; q\right)_{\infty}}{\left(w_{j} m_{f} ; q\right)_{\infty}},
$$

where $m_{\mathrm{a}}$ is the adjoint fugacity, $m_{f}, \bar{m}_{f}$ are the fugacities for the fundamental and antifundamentals and we also turned on the Fayet-Iliopoulos (FI) parameter $\kappa_{1}$. Notice that the multiplet content is proper of an $\mathcal{N}=4$ theory, namely the vector and adjoint chiral multiplets form a $\mathcal{N}=4$ vector multiplet, while the fundamental and anti-fundamental chirals form a fundamental hyper multiplet, however supersymmetry is explicitly broken down to $\mathcal{N}=2$ by mass parameters and superpotential terms which we do not discuss.

It was pointed out in [68] (see also the review [69] and [54,96] for the $A D E$ generalization) that the matrix model above manifestly matches a free boson correlator of $q$-Virasoro screening currents and vertex operators. In fact, by using the results reviewed in the previous subsection we can immediately identify ${ }^{6}$

$$
\mathcal{B}_{c}^{3 \mathrm{~d}}=\oint_{c} \mathrm{~d}^{N} \underline{w}\left\langle\alpha_{\infty}\left|\prod_{f=1}^{N_{\mathrm{f}}} \mathrm{H}_{\gamma_{f}}\left(z_{f}\right) \prod_{j=1}^{N} \mathrm{~S}\left(w_{j}\right)\right| \alpha_{0}\right\rangle
$$

up to proportionality factors, provided that we identify

\begin{tabular}{|c|c|c|c|c|c|}
\hline Gauge theory & $q$ & $m_{\mathrm{a}}$ & $m_{f}$ & $\bar{m}_{f}$ & $\kappa_{1}$ \\
\hline \hline$q$-Virasoro & $q$ & $t$ & $q^{\frac{1}{2}} t^{\frac{\gamma_{f}}{2}} z_{f}^{-1}$ & $q^{-\frac{1}{2}} t^{-\frac{\gamma_{f}}{2}} z_{f}^{-1}$ & $\sqrt{\beta}\left(\alpha_{0}+\sqrt{\beta} N-Q_{\beta}\right)$ \\
\hline
\end{tabular}

\footnotetext{
${ }^{6}$ Here we have to use that the $q$-constant $c_{\beta}(\underline{w} ; q)$ in (2.27) simply contributes with a constant multiplicative factor to the integral along the contour chosen in [68].
} 
with $\alpha_{\infty}=\alpha_{0}+2 \sqrt{\beta} N+\sum_{f} \gamma_{f}$. We see that the screening currents provide the vector and adjoint integration measure, while each vertex operator provide the 1-loop potential of a pair of fundamental/anti-fundamental chiral multiplets (the letter $\mathrm{H}$ refers indeed to hyper multiplet, while the half vertex operator $V$ introduced in (2.40) couples a single chiral).

The correlator (2.47) is a particular projection of the state given in (2.32): we have simply to shift the time variables $\tau_{n}$ as in (2.39) and then set $\tau_{n}=0$. Therefore the natural question arises whether we can give a gauge theory interpretation to the more general state (2.32). In order to answer to this question we have to include more observables in the gauge theory. One kind of such observable is given by supersymmetric Wilson loops along the $S^{1}$ at the tip of the disk. ${ }^{7}$ Such insertions contribute to the integral kernel $\Upsilon^{3 \mathrm{~d}}(\underline{w})$ with

$$
\operatorname{Tr}_{\mathcal{R}}(\boldsymbol{w})=s_{\mathcal{R}}(\underline{w})
$$

where $\mathcal{R}$ is an arbitrary $\mathrm{U}(N)$ representation, $s_{\mathcal{R}}(\underline{w})$ is the associated Schur polynomial, $\boldsymbol{w}=\prod_{j} w_{j}^{\boldsymbol{h}^{j}}$ is the Wilson line at the localization locus and $\left\{\boldsymbol{h}^{j}, j=1, \ldots, N\right\}$ are Cartan generators of the gauge group. By using the Cauchy identity

$$
\sum_{\mathcal{R}} s_{\mathcal{R}}(\underline{\hat{\tau}}) s_{\mathcal{R}}(\underline{w})=\mathrm{e}^{-\sum_{j, k} \ln \left(1-\hat{\tau}_{k} w_{j}\right)}=\mathrm{e}^{\sum_{n>0} \tau_{n} \sum_{j} w_{j}^{n}}, \quad \tau_{n}=\sum_{k} \frac{\hat{\tau}_{k}^{n}}{n},
$$

we are naturally led to package Wilson loop v.e.v.'s into the generating function

$$
\begin{aligned}
Z(\underline{\tau}) & =\sum_{\mathcal{R}} s_{\mathcal{R}}(\underline{\hat{\tau}}) \oint_{c} \frac{\mathrm{d}^{N} \underline{w}}{2 \pi \mathrm{i} \underline{w}} \prod_{j=1}^{N} w_{j}^{\kappa_{1}} \prod_{k \neq j=1}^{N} \frac{\left(w_{k} w_{j}^{-1} ; q\right)_{\infty}}{\left(m_{\mathrm{a}} w_{k} w_{j}^{-1} ; q\right)_{\infty}} s_{\mathcal{R}}(\underline{w}) \\
& =\oint_{c} \frac{\mathrm{d}^{N} \underline{w}}{2 \pi \underline{\mathrm{i}} \underline{w}} \Delta_{\beta}(\underline{w}) \mathrm{e}^{\sum_{n>0} \tau_{n} \sum_{j} w_{j}^{n}+\kappa_{1} \sum_{j} \ln w_{j}},
\end{aligned}
$$

which matches (modulo the remark in footnote 6) the highest weight state (2.32) by using the representation (2.31). Our interpretation also implies that the generating function of the theory on $D^{2} \times S^{1}$ satisfies the $q$-Virasoro constraints (2.33).

Starting from the next section, we are going to study $3 \mathrm{~d} \mathcal{N}=2$ gauge theories on compact spaces. We are going to show that the $q$-Virasoro algebra still plays a prominent role, but we have to introduce a new remarkable structure: the modular double.

\section{Gauge Theory on $S_{b}^{3}$}

Supersymmetric gauge theories can be conveniently studied on compact spaces, and $3 \mathrm{~d}$ $\mathcal{N}=2$ YM-CS theories can be placed in a variety of backgrounds while preserving 2 supercharges of opposite R-charge [28-32]. Expectation values of supersymmetric observables computed through Coulomb branch localization provide interesting examples of matrix models, and our goal is to show that we can use $q$-Virasoro/W algebras techniques to study these theories. We will be focusing on single node $\mathrm{U}(N)$ theories coupled to 1 adjoint chiral multiplet and possibly (anti-)fundamental chirals, postponing the discussion of more general unitary quiver theories to Sect. 6. We will not discuss

\footnotetext{
7 Other defects includes boundary degrees of freedom such as walls, see e.g. [56]. We will consider such insertion in Sect. 5 when discussing the inclusion of CS terms.
} 
superpotential terms which cannot be seen by the matrix model except for possible restrictions on the parameters of the theory.

In this section we focus on gauge theories on the squashed $S_{b}^{3}$ geometry [35-38] (see also the review [106]). The $S_{b}^{3}$ can be defined by the usual embedding $S^{3} \subset \mathbb{R}^{4}$ endowed with the metric

$$
\mathrm{d} s^{2}=\omega_{1}^{2}\left(\mathrm{~d} x_{1}^{2}+\mathrm{d} x_{2}^{2}\right)+\omega_{2}^{2}\left(\mathrm{~d} x_{3}^{2}+\mathrm{d} x_{4}^{2}\right), \quad b^{2}=\frac{\omega_{2}}{\omega_{1}}, \quad \omega=\omega_{1}+\omega_{2},
$$

where $\omega_{1,2}$ are squashing parameters. ${ }^{8}$ For our purposes it is useful to keep in mind that $S_{b}^{3}$ can be obtained by gluing two solid tori $D^{2} \times S^{1}$ through the $S \in \operatorname{SL}(2, \mathbb{Z})$ element acting on the boundary torus with modular parameter $\epsilon$, namely

$$
q=\mathrm{e}^{2 \pi \mathrm{i} \epsilon} \rightarrow \mathrm{e}^{-2 \pi \mathrm{i} S \cdot \epsilon}, \quad \epsilon \rightarrow S \cdot \epsilon=-\frac{1}{\epsilon} .
$$

3.1. Generating function. Coulomb branch localization implies that the path integral localizes onto trivial field configurations except for a constant profile of the adjoint vector multiplet scalar $\boldsymbol{X}=\sum_{j} X_{j} \boldsymbol{h}^{j}$ in the Cartan subalgebra, which is to be integrated over. If we consider the YM-CS theory coupled to 1 adjoint chiral multiplet of complexified mass $M_{\mathrm{a}}{ }^{9}$ the partition function of the theory is given by ${ }^{10}$

$$
\begin{aligned}
Z & =\mathcal{N}_{0} \int_{\mathrm{i}_{\mathbb{R}^{N}}} \mathrm{~d}^{N} \underline{X} \Delta_{S}(\underline{X}) \mathrm{e}^{\sum_{j} V\left(X_{j}\right)}, \\
\Delta_{S}(\underline{X}) & =\prod_{k \neq j} \frac{S_{2}\left(X_{k}-X_{j} \mid \underline{\omega}\right)}{S_{2}\left(M_{\mathrm{a}}+X_{k}-X_{j} \mid \underline{\omega}\right)},
\end{aligned}
$$

where $\Delta_{S}(\underline{X)}$ is the integration measure capturing the vector and adjoint contributions and the double Sine function is defined in (A.10). The potential $V(X)$ is determined by the classical CS action (including the FI)

$$
V(X)=-\frac{i \pi \kappa_{2}}{\omega_{1} \omega_{2}} X^{2}+\frac{2 \pi i \kappa_{1}}{\omega_{1} \omega_{2}} X,
$$

where $\kappa_{2}$ is the CS level and $\kappa_{1}$ is the FI parameter, while $\mathcal{N}_{0}$ is an overall normalization constant. Notice that the theory with $\kappa_{2}=\kappa_{1}=0$ is a.k.a. the $\mathcal{N}=2^{*}$ theory, namely the pure $\mathcal{N}=4$ YM theory broken down to $\mathcal{N}=2$ by the adjoint mass.

A relevant class of observables which can be computed though localization is provided by supersymmetric Wilson loops in arbitrary representations of the gauge group, which can be inserted along the great circles $x_{3}=x_{4}=0$ (length $\left.2 \pi / \omega_{1}\right)$ or $x_{1}=x_{2}=0$ (length $\left.2 \pi / \omega_{2}\right)$ at the North or South poles of the Hopf base (see for instance [108]). Such insertions amount to evaluate the v.e.v. of

$$
\operatorname{Tr}_{\mathcal{R}_{i}}\left(\mathrm{e}^{\frac{2 \pi \mathrm{i}}{\omega_{i}} X}\right)=s_{\mathcal{R}_{i}}\left(\mathrm{e}^{\frac{2 \pi \mathrm{i}}{\omega_{i}} \underline{X}}\right),
$$

\footnotetext{
${ }^{8}$ In this parametrization the squashing parameters are assumed to be real. However, once the gauge theory observables are computed by localization, $\omega_{1,2}$ can be taken to be complex.

9 The real mass is $M_{\mathrm{a}}^{\mathbb{R}}=\mathrm{i} M_{\mathrm{a}}-\mathrm{i} \frac{\omega}{2} \Delta$, where $\Delta$ is the Weyl dimension. The latter is absorbed into the complex mass due to holomorphy [107].

10 To compare with the literature we have to use $S_{2}(\omega / 2-\mathrm{i} X \mid \underline{\omega})=s_{b}(X)$, where $\omega_{1}=\omega_{2}^{-1}=b$.
} 
for an arbitrary $\mathrm{U}(N)$ representation $\mathcal{R}_{i}$. Using the identity (2.50) we are naturally led to package Wilson loop v.e.v.'s into the generating function

$$
\begin{aligned}
& Z\left(\underline{\tau}_{1}, \underline{\tau}_{2}\right)=\sum_{\mathcal{R}_{1}, \mathcal{R}_{2}} \mathcal{N}_{0} \int_{i \mathbb{R}^{N}} \mathrm{~d}^{N} \underline{X} \Delta_{S}(\underline{X}) \mathrm{e}^{\sum_{j} V\left(X_{j}\right)} \prod_{i=1,2} s_{\mathcal{R}_{i}}\left(\underline{\hat{\tau}}_{i}\right) s_{\mathcal{R}_{i}}\left(\mathrm{e}^{\frac{2 \pi \mathrm{i}}{\omega_{i}} \underline{X}}\right) \\
& =\int_{\mathrm{i} \mathbb{R}^{N}} \mathrm{~d}^{N} \underline{X} \Delta_{S}(\underline{X}) \mathrm{e}^{\sum_{j} V\left(X_{j}\right)} \prod_{i=1,2} \exp \left(\sum_{n>0} \tau_{n, i} \sum_{j} \mathrm{e}^{\frac{2 \pi \mathrm{in} n}{\omega_{i}} X_{j}}+\tau_{0, i} N \kappa_{0}\right),
\end{aligned}
$$

where we parametrized $\mathcal{N}_{0}=\exp N \kappa_{0}\left(\tau_{0,1}+\tau_{0,2}\right)$. Therefore, the inclusion of Wilson loops modifies the matrix model potential according to

$$
V(X) \rightarrow V\left(X \mid \underline{\tau}_{1}, \underline{\tau}_{2}\right)=V(X)+\sum_{i=1,2}\left(\sum_{n>0} \tau_{n, i} \mathrm{e}^{\frac{2 \pi \mathrm{i} n}{\omega_{i}} X}+\tau_{0, i} \kappa_{0}\right)
$$

Notice that the partition function $Z$ is simply $Z(\underline{0}, \underline{0})$. However, the generating function is a much more interesting object to study as it contains more information than the bare partition function. Moreover, the contribution of (anti-)fundamental matter can be included as a background for the time variables and CS levels, which is the reason why we will be mostly interested in the theory without (anti-)fundamental matter. In fact, a fundamental chiral multiplet of complexified mass $M_{\mathrm{f}}$ can be coupled to the theory by adding the following 1-loop term to the potential

$$
V(X)_{\text {fund. }}=-\ln S_{2}\left(X+M_{\mathrm{f}} \mid \underline{\omega}\right) .
$$

On the other hand, for generic squashing parameters (i.e. $\left.\operatorname{Im}\left(\omega_{2} / \omega_{1}\right) \neq 0\right)$ the double Sine function has the representation (A.13)

$$
-\ln S_{2}(X \mid \underline{\omega})=-\frac{\mathrm{i} \pi}{2 \omega_{1} \omega_{2}}\left(X^{2}-X \omega+\frac{\omega^{2}+\omega_{1} \omega_{2}}{6}\right)+\sum_{i=1,2} \sum_{n>0} \frac{\mathrm{e}^{\frac{2 \pi \mathrm{in}}{\omega_{i}} X}}{n\left(1-\mathrm{e}^{2 \pi \mathrm{i} n \frac{\omega}{\omega_{i}}}\right)} .
$$

It is therefore clear that a fundamental chiral multiplet can be simply coupled to the theory by shifting the time variables and CS levels according to

$$
\kappa_{2} \rightarrow \kappa_{2}+\frac{1}{2}, \quad \kappa_{1} \rightarrow \kappa_{1}+\frac{\omega}{4}-\frac{M_{\mathrm{f}}}{2}, \quad \tau_{n, i} \rightarrow \tau_{n, i}+\frac{\mathrm{e}^{\frac{2 \pi \mathrm{i} n}{\omega_{i}} M_{\mathrm{f}}}}{n\left(1-\mathrm{e}^{2 \pi \mathrm{i} n \frac{\omega}{\omega_{i}}}\right)}, \quad n>0
$$

and $\ln \mathcal{N}_{0} \rightarrow \ln \mathcal{N}_{0}-\frac{\mathrm{i} \pi}{2 \omega_{1} \omega_{2}}\left(M_{\mathrm{f}}^{2}-M_{\mathrm{f}} \omega-\frac{\omega_{1}^{2}+\omega_{2}^{2}-3 \omega^{2}}{12}\right)$. It is worth noting that the shift of the time variables alone corresponds to gauging a tetrahedron theory $[12,13]$ for each weight, which automatically gets rid of the parity anomaly due to half-integer CS units.

In the next subsection we are going to show that the generating function (3.7) has a neat $q$-Virasoro interpretation and that it satisfies two commuting copies of $q$-Virasoro constraints. 
3.2. Free boson realization. To begin with, let us consider the matrix model corresponding to the gauge theory generating function (3.7) without pure CS action, i.e. $\kappa_{2}=0$. We discuss the inclusion of CS terms in Sect. 5. As reviewed in Sect. 2, the simplest strategy to derive the constraints satisfied by the matrix model is to look for its free boson realization. We can in fact give such representation by means of two commuting copies of the very same Heisenberg algebra (2.22) (we display non-trivial relations only)

$$
\begin{gathered}
{\left[\mathrm{a}_{n, i}, \mathrm{a}_{m, i}\right]=\frac{1}{n}\left(q_{i}^{\frac{n}{2}}-q_{i}^{-\frac{n}{2}}\right)\left(t_{i}^{\frac{n}{2}}-t_{i}^{-\frac{n}{2}}\right)\left(p_{i}^{\frac{n}{2}}+p_{i}^{-\frac{n}{2}}\right) \delta_{n+m, 0},} \\
{\left[\mathrm{P}_{i}, \mathrm{Q}_{i}\right]=2, n, m \in \mathbb{Z} \backslash\{0\},}
\end{gathered}
$$

where the subindex $i=1,2$ denotes the two copies. The $q$-Virasoro and gauge theory parameters are related by

\begin{tabular}{|c|l|l|}
\hline Gauge & \multicolumn{1}{|c|}{$\omega_{1}, \omega_{2}, M_{\mathrm{a}}$} \\
\hline \hline \multirow{4}{*}{$q$-Virasoro } & \multicolumn{1}{|c|}{ Copy 1 } & \multicolumn{1}{|c|}{ Copy 2 } \\
\cline { 2 - 3 } & $q_{1}=\mathrm{e}^{2 \pi \mathrm{i} \frac{\omega}{\omega_{1}}}$ & $q_{2}=\mathrm{e}^{2 \pi \mathrm{i} \frac{\omega}{\omega_{2}}}$ \\
& $t_{1}=\mathrm{e}^{2 \pi \mathrm{i} \frac{\beta_{1} \omega}{\omega_{1}}}=\mathrm{e}^{\frac{2 \pi \mathrm{i}}{\omega_{1}} M_{\mathrm{a}}}$ & $t_{2}=\mathrm{e}^{2 \pi \mathrm{i} \frac{\beta_{2} \omega}{\omega_{2}}}=\mathrm{e}^{\frac{2 \pi \mathrm{i}}{\omega_{2}} M_{\mathrm{a}}}$ \\
& $\beta_{1}=\beta$ & $\beta_{2}=\beta$ \\
\hline
\end{tabular}

By introducing the fundamental weight variables (i.e. the Wilson lines evaluated on the $j$ th fundamental weight)

$$
\left(w_{j}\right)_{1}=\mathrm{e}^{\frac{2 \pi \mathrm{i}}{\omega_{1}} X_{j}}, \quad\left(w_{j}\right)_{2}=\mathrm{e}^{\frac{2 \pi \mathrm{i}}{\omega_{2}} X_{j}},
$$

the integration measure (3.4) is reproduced by the current

$$
\mathcal{S}(X)=(w)_{1}(w)_{2} \mathbf{S}(w)_{1} \otimes \mathbf{S}(w)_{2},
$$

which is essentially the product of two commuting $q$-Virasoro screening currents defined in (2.24). In fact

$$
\prod_{j} \mathcal{S}\left(X_{j}\right)=: \prod_{j} \mathrm{~S}\left(w_{j}\right)_{1} \otimes \mathrm{S}\left(w_{j}\right)_{2}: \Delta_{S}(\underline{X}) \mathrm{e}^{\frac{2 \pi \mathrm{i} \omega \sqrt{\beta}}{\omega_{1} \omega_{2}}\left(\sqrt{\beta} N-Q_{\beta}\right) \sum_{j} X_{j}},
$$

where we recall the definition $Q_{\beta}=\sqrt{\beta}-1 / \sqrt{\beta}$. Here we used (2.27), the representation (A.13) of the double Sine function and the modular property (A.6) of the $\Theta$ function with $\epsilon=\omega / \omega_{1}, r=1$. Table (3.13) summarizes the $g \in \operatorname{SL}(2, \mathbb{Z})$ gluing involved in our construction

$$
\epsilon \rightarrow g \cdot \epsilon=\frac{\epsilon}{1-\epsilon} \Rightarrow q_{1}=\mathrm{e}^{2 \pi \mathrm{i} \epsilon}, \quad q_{2}=\mathrm{e}^{-2 \pi \mathrm{i} g \cdot \epsilon}
$$

which nicely reflects the geometric decomposition of $S_{b}^{3}$ into a pair of solid tori $D^{2} \times S^{1}$ each equipped with its own copy of the $q$-Virasoro algebra. Moreover, by parametrizing $X / \omega_{1}=\chi$ we also have the $g \in \operatorname{SL}(2, \mathbb{Z})$ action on the coordinates

$$
\chi \rightarrow g \cdot \chi=\frac{\chi}{1-\epsilon} \Rightarrow(w)_{1}=\mathrm{e}^{2 \pi \mathrm{i} \chi}, \quad(w)_{2}=\mathrm{e}^{-2 \pi \mathrm{i} g \cdot \chi}
$$


We should notice that the above parametrization is adapted to the lens space description $S_{b}^{3} \simeq L(1,1)$ of the next section, but we can recover the more familiar description through the $S \in \operatorname{SL}(2, \mathbb{Z})$ gluing by identifying $\epsilon \simeq \epsilon+1$.

We next define the operator

$$
\mathcal{Z}=\mathcal{J}^{N}, \quad \mathcal{J}=\int_{\text {i }} \mathrm{d} X \mathcal{S}(X)
$$

built from the screening charge $\mathcal{J}$. When acting on the charged Fock vacuum state $|\alpha\rangle$ defined by

$$
|\alpha\rangle=\mathrm{e}^{\frac{\alpha}{2} \mathrm{Q}_{1}} \otimes \mathrm{e}^{\frac{\alpha}{2} \mathrm{Q}_{2}}|0\rangle, \quad \mathrm{a}_{n, i}|0\rangle=0, \quad \mathrm{P}_{i}|\alpha\rangle=\alpha|\alpha\rangle, \quad n>0,
$$

we get

$$
\begin{aligned}
\mathcal{Z}|\alpha\rangle= & \int_{i \mathbb{R}^{N}} \mathrm{~d}^{N} \underline{X} \Delta_{S}(\underline{X}) \mathrm{e}^{\frac{2 \pi \mathrm{i} \omega \sqrt{\beta}}{\omega_{1} \omega_{2}}\left(\alpha+\sqrt{\beta} N-Q_{\beta}\right) \sum_{j} X_{j}} \\
& \times \bigotimes_{i=1,2} \exp \left(\sum_{n>0} \frac{\sum_{j}\left(w_{j}^{n}\right)_{i}}{q_{i}^{n / 2}-q_{i}^{-n / 2}} \mathrm{a}_{-n, i}\right) \mathrm{e}^{\sqrt{\beta} N \mathrm{Q}_{i}}|\alpha\rangle .
\end{aligned}
$$

Using the algebra representation (2.31) we can finally write

$$
\begin{aligned}
\mathcal{Z}|\alpha\rangle \simeq Z\left(\underline{\tau}_{1}, \underline{\tau}_{2}\right)= & \int_{\mathrm{i} \mathbb{R}^{N}} \mathrm{~d}^{N} \underline{X} \Delta_{S}(\underline{X}) \mathrm{e}^{\frac{2 \pi \mathrm{i} \omega \sqrt{\beta}}{\omega_{1} \omega_{2}}\left(\alpha+\sqrt{\beta} N-Q_{\beta}\right) \sum_{j} X_{j}} \\
& \times \prod_{i=1,2} \exp \left(\sum_{n>0} \tau_{n, i} \sum_{j} \mathrm{e}^{\frac{2 \pi \mathrm{in}}{\omega_{i}} X_{j}}+\tau_{0, i}\left(N+\frac{\alpha}{2 \sqrt{\beta}}\right)\right),
\end{aligned}
$$

matching the gauge theory generating function provided that we identify

$$
\kappa_{1}=\omega \sqrt{\beta}\left(\alpha+\sqrt{\beta} N-Q_{\beta}\right), \quad \kappa_{0}=1+\frac{\alpha}{2 N \sqrt{\beta}} .
$$

In order to show that the matrix model (3.22) satisfies two commuting copies of $q$-Virasoro constraints, we have to verify that the $q$-Virasoro generators $\mathrm{T}_{n, i}$ in the free boson representation (3.12) commute with the screening current (3.15) up to total differences, namely

$$
\left[\mathrm{T}_{n, i}, \mathcal{S}(X)\right]=\mathcal{O}\left(\lambda_{i}+X\right)_{i}-\mathcal{O}(X)_{i}
$$

for some ( $n$-dependent) operator $\mathcal{O}(X)_{i}$ and $\lambda_{i} \in \mathbb{C}$. Indeed, assuming that $\sqrt{\beta} \mathrm{P}_{i}$ has integer eigenvalues, ${ }^{11}$ we can use the $\omega_{i}$-periodicity in the $i^{\text {th }}$ copy and the relation $(2.25)$ valid for the two copies

$$
\begin{aligned}
& {\left[\mathrm{T}_{n, 1}, \mathrm{~S}(w)_{i}\right]=(w)_{i}^{-1}\left(\mathrm{O}\left(q_{i} w\right)_{i}-\mathrm{O}(w)_{i}\right) \delta_{1, i},} \\
& {\left[\mathrm{~T}_{n, 2}, \mathrm{~S}(w)_{i}\right]=(w)_{i}^{-1}\left(\mathrm{O}\left(q_{i} w\right)_{i}-\mathrm{O}(w)_{i}\right) \delta_{2, i},}
\end{aligned}
$$

to conclude that (3.24) holds true with

$\mathcal{O}(X)_{1}=(w)_{2} \mathrm{O}(w)_{1} \otimes \mathrm{S}(w)_{2}, \quad \mathcal{O}(X)_{2}=(w)_{1} \mathrm{~S}(w)_{1} \otimes \mathrm{O}(w)_{2}, \quad \lambda_{1,2}=\omega_{2,1}$.

\footnotetext{
11 We can relax this constraint by modifying the zero modes without affecting the algebra.
} 
This non-trivial property of the screening current $\mathcal{S}(X)$ and the $\operatorname{SL}(2, \mathbb{Z})$ pairing justify the name "modular double" that we are using for our construction. Finally, from the interpretation (3.22) and the highest weight condition

$$
\mathrm{T}_{n, i}|\alpha\rangle=0, \quad n>0,
$$

we easily get $q$-Virasoro constraints through the representation (2.31) of (2.33)

$$
T\left(z \mid \underline{\tau}_{i}\right)_{i} Z\left(\underline{\tau}_{1}, \underline{\tau}_{2}\right)=\operatorname{Pol}(z)_{i} \Rightarrow T_{n, i}\left(\underline{\tau}_{i}\right) Z\left(\underline{\tau}_{1}, \underline{\tau}_{2}\right)=0, \quad n>0,
$$

for a certain $\left(\tau_{i}\right.$-dependent) polynomial $\operatorname{Pol}(z)_{i}$ and where $T_{n, i}\left(\underline{\tau}_{i}\right)$ are the differential operators given in (2.34).

3.3. Round $S^{3}: W_{1, t}$ and Virasoro limits. The $q$-Virasoro $=\mathrm{W}_{q, t}\left(A_{1}\right)$ algebra admits several interesting limits in which it reduces to other known algebras, the most famous one being the conformal limit discussed around (2.21). Other interesting limits are: the Hall-Littlewood limit $q \rightarrow 0$ with $t$ fixed [109] and recently discussed in [110] in the context of the 5d AGT correspondence; the root of unity limit [111] recently discussed in the context of the $4 \mathrm{~d}$ AGT correspondence in [112,113]; the special values $\beta=1,3 / 2,2$ in which case connections with Kac-Moody, topological and $\mathrm{W}_{1+\infty}$ algebras respectively were discussed in [109]; the Frenkel-Reshetikhin limit $t \rightarrow 1$ with $q$ fixed (classical $q$-Virasoro algebra) or $q \rightarrow 1$ with $t$ fixed [114], in which case the algebra becomes commutative but inherits a natural Poisson algebra structure isomorphic to the Poisson algebra obtained from the difference Drinfeld-Sokolov reduction of $\widehat{\mathrm{SL}}_{2}[115,116]$.

It would be very interesting to understand all these limits from the viewpoint of the $q$-Virasoro modular double and $3 \mathrm{~d}$ gauge theories on compact spaces, but the general discussion is beyond the aim of this work. Also, we should observe that taking the limits at the algebra level might be very subtle: in fact, it may happen that a particular (naive) limit on one $q$-Virasoro factor is ill-defined on the other. In the following we will simply ignore these subtleties and study instead a couple of particular limits where the compact space generating function is perfectly defined and allows us to explicitly find the constraints it satisfies by standard matrix model techniques. For concreteness, we will focus on the $S_{b}^{3}$ geometry discussed in this section, analyzing the matrix model (3.22) in special limits of the deformation parameters. First of all, we consider the round $S^{3}$ limit corresponding to $\omega_{1} \rightarrow \omega_{2} \rightarrow 1$ from a complex direction. In terms of the parameters of the $q$-Virasoro algebra this limit corresponds to $q_{1}^{1 / 2} \rightarrow q_{2}^{1 / 2} \rightarrow 1$. However, in this limit the value of $t_{1}^{1 / 2} \rightarrow t_{2}^{1 / 2} \rightarrow \mathrm{e}^{2 \pi \mathrm{i} \beta}$ is still a free parameter and each copy of the $q$-Virasoro algebra should reduce to the $\mathrm{W}_{1, t}\left(A_{1}\right)$ algebra mentioned above. In order to be able to study the matrix model exactly, we can take a further limit on $\beta$ such that $t_{1} \rightarrow t_{2} \rightarrow \pm 1$, in which case we expect to find a relation with the Virasoro algebra (a different one w.r.t. (2.21) though).

$\beta \in \frac{\mathbb{Z}}{2}$ or $t=1$ : Virasoro limit. The first simple example is when $\beta \in \frac{\mathbb{Z}}{2}$, in which case $t_{1,2} \rightarrow t=\mathrm{e}^{4 \pi i \beta} \rightarrow 1$. In this limit the measure (3.4) of the matrix model (3.22) reduces to the following expression

$$
\Delta_{S}(\underline{X})=\left((-1)^{\beta(2 \beta-1)} 2^{2 \beta}\right)^{N(N-1)} \prod_{k \neq j} \sin ^{2 \beta}\left(\pi\left(X_{k}-X_{j}\right)\right),
$$

which can be derived from the reflection property (A.12). Therefore the matrix model becomes 


$$
\begin{aligned}
Z(\underline{\tau})= & \int_{i \mathbb{R}^{N}} \mathrm{~d}^{N} \underline{X} \prod_{k \neq j} \sin ^{2 \beta}\left(\pi\left(X_{k}-X_{j}\right)\right) \\
& \times \mathrm{e}^{2 \pi \mathrm{i} \kappa_{1} \sum_{j} X_{j}} \exp \left(\sqrt{2 \beta} \sum_{n>0} \sum_{j} \tau_{n} \mathrm{e}^{2 \pi \mathrm{i} n X_{j}}+\sqrt{2 \beta} \tau_{0} N \kappa_{0}\right),
\end{aligned}
$$

where we set $\sqrt{2 \beta} \tau_{n}=\tau_{n, 1}+\tau_{n, 2}$ and neglected an overall prefactor which is not relevant for our further discussions. Introducing the exponentiated variables $x_{j}=\mathrm{e}^{2 \pi \mathrm{i} X_{j}}$, we can rewrite the matrix model as

$$
Z(\underline{\tau})=\int_{0}^{\infty} \mathrm{d}^{N} \underline{x} \prod_{j} x_{j}^{(1+2 \sqrt{\beta} \alpha)} \prod_{k \neq j}\left(x_{k}-x_{j}\right)^{2 \beta} \mathrm{e}^{\sqrt{2 \beta}\left(\sum_{n>0} \tau_{n} \sum_{j} x_{j}^{n}+\tau_{0} N \kappa_{0}\right)},
$$

up to unimportant proportionality factors, and where we used $\kappa_{1}=2 \sqrt{\beta}\left(\sqrt{\beta} N-Q_{\beta}+\alpha\right)$. Now we can derive the constraints for this matrix integral using standard techniques (for details we refer to [117-119]). We shift the integration variables $x_{j} \rightarrow x_{j}+\varepsilon_{n} x_{j}^{n+1}$, $n \in \mathbb{Z}_{>0}$, and collect all the variations under the integral, leading to the following Ward identities

$$
\begin{aligned}
& \left\langle\sum_{k \geqslant 1} \sqrt{2 \beta} \tau_{k} k \sum_{j} x_{j}^{n+k}+2 \beta \sum_{k=1}^{n-1} \sum_{\ell, j} x_{\ell}^{k} x_{j}^{n-k}\right. \\
& \left.\quad+(4 \beta N+2 \sqrt{\beta} \alpha+1+(n+1)(1-2 \beta)) \sum_{j} x_{j}^{n}\right\rangle=0
\end{aligned}
$$

where \langle\rangle denotes the matrix model average. Such identities are equivalent to the differential constraints $L_{n}(\underline{\tau}) Z(\underline{\tau})=0$ where the $L_{n}(\underline{\tau})$ operators are

$L_{n}(\underline{\tau})=\sum_{k \geqslant 1} \tau_{k} k \frac{\partial}{\partial \tau_{n+k}}+\sum_{k=1}^{n-1} \frac{\partial^{2}}{\partial \tau_{k} \partial \tau_{n-k}}+\left(2 \sqrt{2 \beta} N+\sqrt{2} \alpha+\frac{1}{\sqrt{2 \beta}}-(n+1) Q_{2 \beta}\right) \frac{\partial}{\partial \tau_{n}}$.

We can also use

$$
2 \frac{\partial}{\partial \tau_{0}} Z(\underline{\tau})=2 \sqrt{2 \beta} N \kappa_{0} Z(\underline{\tau}), \quad \kappa_{0}=1+\frac{\alpha}{2 N \sqrt{\beta}},
$$

to rewrite

$$
L_{n}(\underline{\tau})=\sum_{k \geqslant 1} \tau_{k} k \frac{\partial}{\partial \tau_{n+k}}+\sum_{k=1}^{n-1} \frac{\partial^{2}}{\partial \tau_{k} \partial \tau_{n-k}}+\left(2 \frac{\partial}{\partial \tau_{0}}+\frac{1}{\sqrt{2 \beta}}-(n+1) Q_{2 \beta}\right) \frac{\partial}{\partial \tau_{n}} .
$$

These differential operators represent the Virasoro operators (2.8) with zero mode $\mathrm{P} \simeq$ $2 \frac{\partial}{\partial \tau_{0}}+\frac{1}{\sqrt{2 \beta}}$ and central charge $c=1-6 Q_{2 \beta}^{2}$.

$\beta \in \frac{1}{4}+\frac{\mathbb{Z}}{2}$ or $t=-1$ : another Virasoro limit. Another situation in which we can explicitly derive the constraints satisfied by the matrix model is when $\beta \in \frac{1}{4}+\frac{\mathbb{Z}}{2}$. This case corresponds to $t_{1,2} \rightarrow t=\mathrm{e}^{4 \pi \mathrm{i} \beta} \rightarrow-1$. In order to find the limit of the measure (3.4) of the matrix model (3.22) we use 


$$
\frac{S_{2}\left(X+\frac{1}{2} \mid 1,1\right)}{S_{2}\left(X+n+\frac{1}{2} \mid 1,1\right)}=(-1)^{\frac{n(n-1)}{2}} 2^{n} \cos ^{n}(\pi X), \quad n=2 \beta-\frac{1}{2} \in \mathbb{Z},
$$

and the reflection property (A.12), leading the following matrix model

$$
\begin{aligned}
Z(\underline{\tau})= & \int_{i \mathbb{R}^{N}} \mathrm{~d}^{N} \underline{X} \prod_{k<j} \sin ^{2}\left(\pi\left(X_{k}-X_{j}\right)\right) \cos ^{(4 \beta-2)}\left(\pi\left(X_{k}-X_{j}\right)\right) \\
& \times \mathrm{e}^{2 \pi \mathrm{i} \kappa_{1} \sum_{j} X_{j}} \exp \left(\sqrt{2 \beta} \sum_{n>0} \sum_{j} \tau_{n} e^{2 \pi i n X_{j}}+\sqrt{2 \beta} \tau_{0} N \kappa_{0}\right),
\end{aligned}
$$

up to proportionality factors, and where we set $\sqrt{2 \beta} \tau_{n}=\tau_{n, 1}+\tau_{n, 2}$. Rewriting the matrix model in terms of the $x_{j}=\mathrm{e}^{2 \pi \mathrm{i} X}{ }_{j}$ variables we obtain

$$
Z(\underline{\tau})=\int_{0}^{\infty} \mathrm{d}^{N} \underline{x} \prod_{j} x_{j}^{(1+2 \alpha \sqrt{\beta})} \prod_{k<j}\left(x_{k}-x_{j}\right)^{2}\left(x_{k}+x_{j}\right)^{2(2 \beta-1)} \mathrm{e}^{\sqrt{2 \beta}\left(\sum_{n>0} \tau_{n} \sum_{j} x_{j}^{n}+\tau_{0} N \kappa_{0}\right)},
$$

up to proportionality factors, and where we used $\kappa_{1}=2 \sqrt{\beta}\left(\sqrt{\beta} N-Q_{\beta}+\alpha\right)$. Performing the shift $x_{j} \rightarrow x_{j}+\varepsilon_{n} x_{j}^{n+1}, n \in 2 \mathbb{Z}_{>0}$, and collecting all the variations under the integral we arrive at the Ward identities

$$
\begin{aligned}
& \left\langle\sqrt{2 \beta} \sum_{k \geqslant 1} \tau_{k} k \sum_{j} x_{j}^{n+k}+\sum_{k=0}^{n}\left(1+(-1)^{k}(2 \beta-1)\right)\right. \\
& \left.\times \sum_{\ell, j} x_{\ell}^{k} x_{j}^{n-k}+(2 \sqrt{\beta} \alpha+2-2 \beta) \sum_{j} x_{j}^{n}\right\rangle=0 .
\end{aligned}
$$

The reason why these Ward identities can be derived for even $n$ only is that the variation of $\prod_{k \neq j}\left(x_{k}+x_{j}\right)^{2 \beta-1}$ contains

$$
\sum_{k \neq j} \frac{x_{k}^{n+1}+x_{j}^{n+1}}{x_{k}+x_{j}}=\sum_{k=0}^{n} \sum_{\ell \neq j}(-1)^{k} x_{\ell}^{k} x_{j}^{n-k}, \quad n \in 2 \mathbb{Z},
$$

while for odd $n$ a similar simplification to the r.h.s. does not take place. The derived Ward identities can be generated by the action of the following differential operators on the matrix model

$$
\begin{aligned}
L_{n}(\underline{\tau})= & \sum_{k \geqslant 1} \tau_{k} k \frac{\partial}{\partial \tau_{n+k}}+\frac{1}{2 \beta} \sum_{k=1}^{n-1}\left(1+(-1)^{k}(2 \beta-1)\right) \\
& \times \frac{\partial^{2}}{\partial \tau_{k} \partial \tau_{n-k}}+\left(2 \frac{\partial}{\partial \tau_{0}}+\sqrt{\frac{2}{\beta}}-2 \sqrt{\frac{\beta}{2}}\right) \frac{\partial}{\partial \tau_{n}} .
\end{aligned}
$$

In order to interpret these operators, we can make the following identification between the differential and free boson operators satisfying the usual Heisenberg algebra $\left[\mathrm{a}_{n}, \mathrm{a}_{m}\right]=$ $2 n \delta_{n+m, 0}$ 


$$
\begin{aligned}
\mathrm{a}_{n} & \simeq 2 \sqrt{\frac{1+(-1)^{n}(2 \beta-1)}{2 \beta}} \frac{\partial}{\partial \tau_{n}}, \quad \mathrm{a}_{-n} \simeq n \sqrt{\frac{2 \beta}{1+(-1)^{n}(2 \beta-1)}} \tau_{n}, \quad n>0, \\
\mathrm{P} & \simeq 2 \frac{\partial}{\partial \tau_{0}}+\frac{2}{\sqrt{2 \beta}}-2 \sqrt{\frac{\beta}{2}} .
\end{aligned}
$$

Under this identification our operators become

$$
L_{n}(\underline{\tau}) \simeq \mathrm{L}_{n}=\frac{1}{4} \sum_{k \neq 0, n}: \mathrm{a}_{n-k} \mathrm{a}_{k}:+\frac{1}{2} \mathrm{a}_{n} \mathrm{P},
$$

satisfying the Virasoro algebra with the central charge $c=1$ when extended to $n \in \mathbb{Z}$.

\section{Other Compact Backgrounds}

In this section we extend our results to other gauge theory backgrounds. Since the analysis will be quite analogous to the previous one for the $S_{b}^{3}$ background, but with the important difference given by non-trivial fundamental groups, we will be more concise in the presentation.

4.1. $L(r, 1)$. The focus of this subsection is on gauge theories on the squashed lens space $L(r, 1)=S_{b}^{3} / \mathbb{Z}_{r}$ [39-43]. The lens space can be defined as the $S_{b}^{3}$ with metric (3.1) and the additional $\mathbb{Z}_{r}$ quotient by the action

$$
x_{1}+\mathrm{i} x_{2} \rightarrow \mathrm{e}^{\frac{2 \pi \mathrm{i}}{r}}\left(x_{1}+\mathrm{i} x_{2}\right), \quad x_{3}+\mathrm{i} x_{4} \rightarrow \mathrm{e}^{-\frac{2 \pi \mathrm{i}}{r}}\left(x_{3}+\mathrm{i} x_{4}\right) .
$$

It is also useful to keep in mind that $L(r, 1)$ can be obtained by gluing two solid tori $D^{2} \times S^{1}$ through the $g_{r} \in \operatorname{SL}(2, \mathbb{Z})$ element acting on the boundary torus with modular parameter $\epsilon$, namely

$$
q=\mathrm{e}^{2 \pi \mathrm{i} \epsilon} \rightarrow \mathrm{e}^{-2 \pi \mathrm{i} g_{r} \cdot \epsilon}, \quad \epsilon \rightarrow g_{r} \cdot \epsilon=\frac{\epsilon}{1-r \epsilon} .
$$

Generating function. Coulomb branch localization implies that the path integral localizes onto flat connections and a constant profile for the adjoint vector multiplet scalar $\boldsymbol{X}$ in the Cartan, which is to be integrated over. Flat connections are classified by $\pi_{1}(L(r, 1)) \simeq \mathbb{Z}_{r}$, and hence labeled by integers $\underline{\ell} \in \mathbb{Z}_{r}^{N}$ which are to be summed over (we consider unordered sequences). If we consider the YM-CS theory coupled to 1 adjoint chiral multiplet of complexified mass $M_{\mathrm{a}}$ the partition function of the theory is given by

$$
Z=\mathcal{N}_{0} \sum_{\underline{\ell} \in \mathbb{Z}_{r}^{N}} \int_{i \mathbb{R}^{N}} \mathrm{~d}^{N} \underline{X} \Delta_{r}(\underline{X}, \underline{\ell}) \mathrm{e}^{\sum_{j} V\left(X_{j}, \ell_{j}\right)},
$$

where

$$
\begin{aligned}
\Delta_{r}(\underline{X}, \underline{\ell}) & =\prod_{k \neq j} \frac{S_{2,-\left(\ell_{k}-\ell_{j}\right)}\left(X_{k}-X_{j} \mid \underline{\omega}\right)}{S_{2,-\left(\ell_{k}-\ell_{j}\right)}\left(M_{\mathrm{a}}+X_{k}-X_{j} \mid \underline{\omega}\right)}, \\
V(X, \ell) & =-\frac{\mathrm{i} \pi \kappa_{2}}{r \omega_{1} \omega_{2}} X^{2}-\frac{\mathrm{i} \pi \kappa_{2}}{r} \ell^{2}+\frac{2 \pi \mathrm{i} \kappa_{1}}{r \omega_{1} \omega_{2}} X .
\end{aligned}
$$


Here $\kappa_{2}$ is the CS level, $\kappa_{1}$ the FI while the generalized double Sine function is defined in (A.14). Supersymmetric Wilson loops can be inserted along the non-contractible cycles at the North and South poles of the Hopf base. Such insertions amount to evaluate the v.e.v. of

$$
\operatorname{Tr}_{\mathcal{R}_{i}}\left(\mathrm{e}^{\frac{2 \pi \mathrm{i}}{r \omega_{i}} \boldsymbol{X}} \mathrm{e}^{s_{i} \frac{2 \pi \mathrm{i}}{r} \ell}\right), \quad s_{1,2}= \pm 1,
$$

where $\mathrm{e}^{ \pm \frac{2 \pi \mathrm{i}}{r} \ell}$ is the holonomy of the gauge connection along the non-contractible cycle. The generating function thus reads as

$$
\begin{gathered}
Z\left(\underline{\tau}_{1}, \underline{\tau}_{2}\right)=\sum_{\underline{\ell} \in \mathbb{Z}_{r}^{N}} \int_{i \mathbb{R}^{N}} \mathrm{~d}^{N} \underline{X} \Delta_{r}(\underline{X}, \underline{\ell}) \mathrm{e}^{\sum_{j} V\left(X_{j}, \ell_{j} \mid \underline{\tau}_{1}, \underline{\tau}_{2}\right)}, \\
V\left(X, \ell \mid \underline{\tau}_{1}, \underline{\tau}_{2}\right)=V(X, \ell)+\sum_{i=1,2}\left(\sum_{n>0} \tau_{n, i} \mathrm{e}^{\frac{2 \pi i n}{r \omega_{i}}\left(X+s_{i} \omega_{i} \ell\right)}+\tau_{0, i} \kappa_{0}\right),
\end{gathered}
$$

where we parametrized $\mathcal{N}_{0}=\mathrm{e}^{N \kappa_{0}\left(\tau_{0,1}+\tau_{0,2}\right)}$. A fundamental chiral multiplet of complexified mass $M_{\mathrm{f}}$ together with $1 / 2 \mathrm{CS}$ units can be coupled to the theory by shifting the time variables according to

$$
\tau_{n, i} \rightarrow \tau_{n, i}+\frac{\mathrm{e}^{\frac{2 \pi \mathrm{i} n}{r \omega_{i}} M_{\mathrm{f}}}}{n\left(1-\mathrm{e}^{2 \pi \mathrm{i} n \frac{\omega}{r \omega_{i}}}\right)} .
$$

4.2. $S^{2} \times S^{1}$ (index). In this subsection we consider gauge theories on the $S^{2} \times S^{1}$ background associated to the superconformal index $[44,45,66]$. The $S^{1}$ period can be parametrized by $q=\mathrm{e}^{\hbar \epsilon}$. It is also useful to keep in mind that $S^{2} \times S^{1}$ can be obtained by gluing two solid tori $D^{2} \times S^{1}$ through the $i d \in \mathrm{SL}(2, \mathbb{Z})$ element acting on the boundary torus with modular parameter $\epsilon$

$$
q=\mathrm{e}^{\hbar \epsilon} \rightarrow \mathrm{e}^{-\hbar i d \cdot \epsilon}, \quad \epsilon \rightarrow i d \cdot \epsilon=\epsilon .
$$

Generating function. Coulomb branch localization implies that the path integral localizes onto monopole configurations on $S^{2}$ labeled by quantized fluxes $\ell$ to be summed over (we consider unordered sequences), and constant gauge holonomy $\boldsymbol{x}$ around $S^{1}$ to be integrated over the maximal torus. The adjoint vector multiplet scalar is also constant and proportional to the flux. If we consider the YM-CS theory coupled to 1 adjoint chiral multiplet with global fugacity $m_{\mathrm{a}}$ the partition function of the theory is

$$
Z=\mathcal{N}_{0} \sum_{\underline{\ell} \in \mathbb{Z}^{N}} \oint_{\mathbb{T}^{N}} \frac{\mathrm{d}^{N} \underline{x}}{2 \pi \underline{\mathrm{i}} \underline{x}} \Delta_{i d}(\underline{x}, \underline{\ell}) \mathrm{e}^{\sum_{j} V\left(x_{j}, \ell_{j}\right)},
$$

where

$$
\begin{aligned}
\Delta_{i d}(\underline{x}, \underline{\ell}) & =\prod_{k<j} m_{\mathrm{a}}^{-\left(\ell_{k}-\ell_{j}\right)}\left(1-x_{k} x_{j}^{-1} q^{\frac{\ell_{k}-\ell_{j}}{2}}\right)\left(1-x_{j} x_{k}^{-1} q^{\frac{\ell_{k}-\ell_{j}}{2}}\right) \times \\
& \times \frac{\left(m_{\mathrm{a}}^{-1} x_{j} x_{k}^{-1} q^{1+\frac{\ell_{k}-\ell_{j}}{2}} ; q\right)_{\infty}\left(m_{\mathrm{a}}^{-1} x_{k} x_{j}^{-1} q^{1+\frac{\ell_{k}-\ell_{j}}{2}} ; q\right)_{\infty}}{\left(m_{\mathrm{a}} x_{k} x_{j}^{-1} q^{\frac{\ell_{k}-\ell_{j}}{2}} ; q\right)_{\infty}\left(m_{\mathrm{a}} x_{j} x_{k}^{-1} q^{\frac{\ell_{k}-\ell_{j}}{2}} ; q\right)_{\infty}},
\end{aligned}
$$




$$
V(x, \ell)=\kappa_{2} \ell \ln x+\kappa_{1} \ln x+\eta_{1} \ell \ln q .
$$

Here $\kappa_{2}$ is the CS level, $\kappa_{1}$ is the FI and we turned on also the holonomy $q^{\eta_{1}}$ for the topological U(1). Supersymmetric Wilson loops can be supported at the poles of $S^{2}$, and their evaluation amounts to computing the average of

$$
\operatorname{Tr}_{\mathcal{R}_{i}}\left(\boldsymbol{x}^{s_{i}} q^{-\frac{\ell}{2}}\right), \quad s_{1,2}= \pm 1
$$

The generating function of Wilson loop v.e.v.'s. is therefore

$$
\begin{aligned}
Z\left(\underline{\tau}_{1}, \underline{\tau}_{2}\right) & =\sum_{\underline{\ell} \in \mathbb{Z}^{N}} \oint_{\mathbb{T}^{N}} \frac{\mathrm{d}^{N} \underline{x}}{2 \pi \underline{\mathrm{i}} \underline{x}} \Delta_{i d}(\underline{x}, \underline{\ell}) \mathrm{e}^{\sum_{j} V\left(x_{j}, \ell_{j} \mid \underline{\tau}_{1}, \underline{\tau}_{2}\right)}, \\
V\left(x, \ell \mid \underline{\tau}_{1}, \underline{\tau}_{2}\right) & =V(x, \ell)+\sum_{n \neq 0} \tau_{n} x^{n} q^{-\frac{\ell}{2}|n|}+\kappa_{0}\left(\tau_{0,1}+\tau_{0,2}\right),
\end{aligned}
$$

where we set $\tau_{n, 1}=\tau_{n}, \tau_{n, 2}=\tau_{-n}$ for $n>0$ and parametrized $\mathcal{N}_{0}=\mathrm{e}^{N \kappa_{0}\left(\tau_{0,1}+\tau_{0,2}\right)}$. A fundamental chiral multiplet of global fugacity $m_{\mathrm{f}}$ together with $1 / 2$ CS units can be coupled to the theory by shifting the time variables

$$
\tau_{n} \rightarrow \tau_{n}+\frac{m_{\mathrm{f}}^{n}}{|n|\left(1-q^{n}\right)}, \quad n \neq 0 .
$$

4.3. $S^{2} \times S^{1}$ (twisted index). In this subsection we consider gauge theories on the Atwisted $S^{2} \times S^{1}$ background leading to the twisted index [46]. The metric is

$$
\mathrm{d} s^{2}=\mathrm{d} \theta^{2}+\sin ^{2} \theta(\mathrm{d} \phi-2 \pi \epsilon \mathrm{d} y)^{2}+\mathrm{d} y^{2},
$$

where $q=\mathrm{e}^{2 \pi \mathrm{i} \epsilon}$ can be interpreted as the angular momentum fugacity. This background is characterized by a flux for the R-symmetry connection. It is also useful to keep in mind that $S^{2} \times S^{1}$ can be obtained by gluing two solid tori $D^{2} \times S^{1}$ through the id $\in \operatorname{SL}(2, \mathbb{Z})$ element acting on the boundary torus with modular parameter $\epsilon$

$$
q=\mathrm{e}^{2 \pi \mathrm{i} \epsilon} \rightarrow \mathrm{e}^{-2 \pi \mathrm{i} i d \cdot \epsilon}, \quad \epsilon \rightarrow i d \cdot \epsilon=\epsilon .
$$

Generating function. Coulomb branch localization implies that the path integral localizes onto monopole configurations on $S^{2}$ labeled by quantized fluxes $\ell$ to be summed over (we consider unordered sequences), and complexified constant gauge holonomy $\boldsymbol{x}$ around $S^{1}$ to be integrated over the maximal torus. If we consider the YM-CS theory coupled to 1 adjoint chiral multiplet with fugacity $v$ and R-charge $R$ the partition function of the theory is given by (we refer to [46] for the J.K. contour)

$$
Z=\mathcal{N}_{0} \sum_{\underline{\ell} \in \mathbb{Z}^{N}} \oint_{\text {J.K. }} \frac{\mathrm{d}^{N} \underline{x}}{2 \pi \underline{\mathrm{i}} \underline{x}} \Delta_{A}(\underline{x}, \underline{\ell}) \mathrm{e}^{\sum_{j} V\left(x_{j}, \ell_{j}\right)},
$$

where 


$$
\begin{aligned}
\Delta_{A}(\underline{x}, \underline{\ell})= & \prod_{k<j}(-1)^{\ell_{k}-\ell_{j}-R} q^{-\frac{\ell_{k}-\ell_{j}}{2}}\left(1-x_{k} x_{j}^{-1} q^{\frac{\ell_{k}-\ell_{j}}{2}}\right)\left(1-x_{j} x_{k}^{-1} q^{\frac{\ell_{k}-\ell_{j}}{2}}\right) \\
& \times \frac{v^{1-R}\left(x_{k} x_{j}^{-1}\right)^{\ell_{k}-\ell_{j}}}{\left(v x_{k} x_{j}^{-1} q^{\frac{1-\left(1+\ell_{k}-\ell_{j}-R\right)}{2}} ; q\right)_{1+\ell_{k}-\ell_{j}-R}\left(v x_{j} x_{k}^{-1} q^{\frac{1-\left(1+\ell_{j}-\ell_{k}-R\right)}{2}} ; q\right)_{1+\ell_{j}-\ell_{k}-R}},
\end{aligned}
$$

$$
V(x, \ell)=\kappa_{2} \ell \ln x+\kappa_{1} \ln x+\eta_{1} \ell \ln q .
$$

Here $\kappa_{2}$ is the CS level, $\kappa_{1}$ the FI and we turned on also the holonomy $q^{\eta_{1}}$ for the topological U(1), while the finite $q$-Pochhammer symbol is defined in (A.3). Supersymmetric Wilson loops wrapping the integral curve of $\partial_{y}+2 \pi \epsilon \partial_{\phi}$ can be supported at the poles of $S^{2}$. The evaluation of a Wilson loop amounts to compute the average of

$$
\operatorname{Tr}_{\mathcal{R}}\left(x q^{s_{i} \frac{\ell}{2}}\right), \quad s_{1,2}= \pm 1 .
$$

The generating function then reads as

$$
\begin{aligned}
Z\left(\underline{\tau}_{1}, \underline{\tau}_{2}\right) & =\sum_{\underline{\ell} \in \mathbb{Z}^{N}} \oint_{\mathrm{J} . \mathrm{K} .} \frac{\mathrm{d}^{N} \underline{x}}{2 \pi \underline{\mathrm{i}} \underline{x}} \Delta_{A}(\underline{x}, \underline{\ell}) \mathrm{e}^{\sum_{j} V\left(x_{j}, \ell_{j} \mid \underline{\tau}_{1}, \underline{\tau}_{2}\right)}, \\
V\left(x, \ell \mid \underline{\tau}_{1}, \underline{\tau}_{2}\right) & =V(x, \ell)+\sum_{n \neq 0} \tau_{n} x^{|n|} q^{-\frac{\ell}{2} n}+\left(\tau_{0,1}+\tau_{0,2}\right) \kappa_{0},
\end{aligned}
$$

where we also set $\tau_{n, 1}=\tau_{n}, \tau_{n, 2}=\tau_{-n}$ for $n>0$ and parametrized $\mathcal{N}_{0}=\mathrm{e}^{N \kappa_{0}\left(\tau_{0,1}+\tau_{0,2}\right)}$. A fundamental chiral multiplet with global fugacity $m_{\mathrm{f}}$ together with $1 / 2 \mathrm{CS}$ units can be coupled to the theory by shifting the time variables

$$
\tau_{n} \rightarrow \tau_{n}+\frac{m_{\mathrm{f}}^{|n|}}{|n|\left(1-q^{n}\right)}, \quad n \neq 0 .
$$

Free boson realization. The matrix models arising from the different backgrounds find a unified description in terms of the Virasoro modular double. Let us start by identifying the geometric parameters, fundamental weight variable $w$ associated to the supersymmetric Wilson loops, the adjoint mass, fugacity or R-charge as

\begin{tabular}{|c|c|c|c|}
\hline$q$-Virasoro & $L(r, 1)$ & $S^{2} \times S^{1}$ (index) & $S^{2} \times S^{1}$ (twisted index) \\
\hline$q_{1}$ & $\mathrm{e}^{2 \pi \mathrm{i} \frac{\omega}{r \omega_{1}}}$ & $\mathrm{e}^{\hbar \epsilon}$ & $\mathrm{e}^{2 \pi \mathrm{i} \epsilon}$ \\
\hline$q_{2}$ & $\mathrm{e}^{2 \pi \mathrm{i} \frac{\omega}{r \omega_{2}}}$ & $\mathrm{e}^{-\hbar \epsilon}$ & $\mathrm{e}^{-2 \pi \mathrm{i} \epsilon}$ \\
\hline$(w)_{1}$ & $\mathrm{e}^{\frac{2 \pi \mathrm{i}}{r} \ell} \mathrm{e}^{\frac{2 \pi \mathrm{i}}{r \omega_{1}} X}$ & $\mathrm{e}^{-\hbar \epsilon \frac{\ell}{2}} x$ & $\mathrm{e}^{-2 \pi \mathrm{i} \epsilon \frac{\ell}{2}} x$ \\
\hline$(w)_{2}$ & $\mathrm{e}^{-\frac{2 \pi \mathrm{i}}{r} \ell} \mathrm{e}^{\frac{2 \pi \mathrm{i}}{r \omega_{2}} X}$ & $\mathrm{e}^{-\hbar \epsilon \frac{\ell}{2}} x^{-1}$ & $\mathrm{e}^{2 \pi \mathrm{i} \epsilon \frac{\ell}{2}} x$ \\
\hline$t_{1}=q_{1}^{\beta_{1}}$ & $\mathrm{e}^{\frac{2 \pi \mathrm{i}}{r \omega_{1}} M_{\mathrm{a}}}$ & $m_{\mathrm{a}}$ & $\mathrm{e}^{2 \pi \mathrm{i} \epsilon \frac{R}{2}} v$ \\
\hline$t_{2}=q_{2}^{\beta_{2}}$ & $\mathrm{e}^{\frac{2 \pi \mathrm{i}}{r \omega_{2}} M_{\mathrm{a}}}$ & $m_{\mathrm{a}}^{-1}$ & $\mathrm{e}^{-2 \pi \mathrm{i} \epsilon \frac{R}{2}} v$ \\
\hline$\left(\beta_{1}, \beta_{2}\right)$ & $(\beta, \beta)$ & $(\beta, \beta)$ & $(\beta, R-\beta)$ \\
\hline
\end{tabular}

This table summarizes the $g \in \mathrm{SL}(2, \mathbb{Z})$ gluings involved in our construction. If we denote $\omega / r \omega_{1}=\epsilon, X / r \omega_{1}=\chi$ for $L(r, 1)$ or $x=\mathrm{e}^{2 \pi \mathrm{i} \chi}$ for $S^{2} \times S^{1}$, then the two copies are related by 


\begin{tabular}{|c|c|c|c|}
\hline$q$-Virasoro & $L(r, 1)$ & $S^{2} \times S^{1}$ (index) & $S^{2} \times S^{1}$ (twisted index) \\
\hline$q_{1}$ & $\mathrm{e}^{2 \pi \mathrm{i} \epsilon}$ & $\mathrm{e}^{\hbar \epsilon}$ & $\mathrm{e}^{2 \pi \mathrm{i} \epsilon}$ \\
\hline$q_{2}$ & $\mathrm{e}^{-2 \pi \mathrm{i} g \cdot \epsilon}$ & $\mathrm{e}^{-\hbar g \cdot \epsilon}$ & $\mathrm{e}^{-2 \pi \mathrm{i} g \cdot \epsilon}$ \\
\hline$(w)_{1}$ & $\mathrm{e}^{\frac{2 \pi \mathrm{i}}{r} \ell} \mathrm{e}^{2 \pi \mathrm{i} \chi}$ & $\mathrm{e}^{-\hbar \epsilon} \frac{\ell}{2} \mathrm{e}^{2 \pi \mathrm{i} \chi}$ & $\mathrm{e}^{-2 \pi \mathrm{i} \epsilon \frac{\ell}{2}} \mathrm{e}^{2 \pi \mathrm{i} \chi}$ \\
\hline$(w)_{2}$ & $\mathrm{e}^{-\frac{2 \pi \mathrm{i}}{r} g \cdot \ell} \mathrm{e}^{-2 \pi \mathrm{i} g \cdot \chi}$ & $\mathrm{e}^{-\hbar g \cdot \epsilon g \cdot \frac{\ell}{2}} \mathrm{e}^{-2 \pi \mathrm{i} g \cdot \chi}$ & $\mathrm{e}^{-2 \pi \mathrm{i} g \cdot \epsilon g \cdot \frac{\ell}{2}} \mathrm{e}^{-2 \pi \mathrm{i} g \cdot \chi}$ \\
\hline$g \cdot \epsilon$ & $\frac{\epsilon}{1-r \epsilon}$ & $\epsilon$ & $\epsilon$ \\
\hline$g \cdot \chi$ & $\frac{\chi}{1-r \epsilon}$ & $\chi$ & $-\chi$ \\
\hline$g \cdot \ell$ & $\ell$ & $\ell$ & $-\ell$ \\
\hline
\end{tabular}
rent

The matrix models are reproduced by the $q$-Virasoro modular double screening cur-

$$
\mathcal{S}(\chi)=\sum_{\ell \in \mathbb{F}}(w)_{1}(w)_{2} \mathbf{S}(w)_{1} \otimes \mathbf{S}(w)_{2},
$$

where we recall that $\mathbb{F}=\left(\mathbb{Z}_{r}, \mathbb{Z}\right)$ for $L(r, 1)$ and $S^{2} \times S^{1}$ respectively. In fact

$$
\prod_{j} \mathcal{S}\left(\chi_{j}\right)=\sum_{\underline{\ell} \in \mathbb{F}^{N}}: \prod_{j} \mathrm{~S}\left(w_{j}\right)_{1} \otimes \mathrm{S}\left(w_{j}\right)_{2}: \Delta(\underline{\chi}, \underline{\ell}) \prod_{j} \Delta_{0}\left(\chi_{j}, \ell_{j}\right),
$$

where the measure $\Delta(\underline{\chi}, \underline{\ell})$ is the one appearing in (4.4), (4.11), (4.19) respectively, and

$$
\Delta_{0}\left(\chi_{j}, \ell_{j}\right)= \begin{cases}\mathrm{e}^{\frac{2 \pi \mathrm{i} \omega \sqrt{\beta}}{r \omega 1} \omega_{2}\left(\sqrt{\beta} N-Q_{\beta}\right) X_{j}} & : L(r, 1) \\ q^{-\sqrt{\beta} \ell_{j}\left(\sqrt{\beta} N-Q_{\beta}\right)} & : S^{2} \times S^{1} \text { (index) } \\ x_{j}^{R(N-1)+2} q^{-\ell_{j}\left(\beta-\frac{R}{2}\right)(N-1)} & : S^{2} \times S^{1} \text { (twisted index) }\end{cases}
$$

Here we used (2.27), (2.28), (A.15), (A.6), (A.2), (A.4). We next define the operator

$$
\mathcal{Z}=\mathcal{J}^{N}, \quad \mathcal{J}=\int_{\mathrm{i} \mathbb{R}} \mathrm{d} \chi \mathcal{S}(\chi)
$$

yielding the state

$$
\begin{aligned}
\mathcal{Z}|\alpha\rangle= & \sum_{\underline{\ell} \in \mathbb{F}^{N}} \int \mathrm{d}^{N} \underline{\chi} \Delta(\underline{\chi}, \underline{\ell}) \prod_{j} \Delta_{0}\left(\chi_{j}, \ell_{j}\right)\left(w_{j}\right)_{1}^{\sqrt{\beta} \alpha}\left(w_{j}\right)_{2}^{\sqrt{\beta} \alpha} \\
& \times \bigotimes_{i=1,2} \exp \left(\sum_{n>0} \frac{\sum_{j}\left(w_{j}^{n}\right)_{i}}{q_{i}^{n / 2}-q_{i}^{-n / 2}} \mathrm{a}_{-n, i}\right) \mathrm{e}^{\sqrt{\beta} N \mathrm{Q}_{i}}|\alpha\rangle .
\end{aligned}
$$

Using the algebra representation (2.31) we can finally match (4.7), (4.14), (4.22) identifying

\begin{tabular}{|l|c|c|c|}
\hline & $L(r, 1)$ & $S^{2} \times S^{1}$ (index) & $S^{2} \times S^{1}$ (twisted index) \\
\hline$\kappa_{2}$ & 0 & 0 & 0 \\
\hline$\kappa_{1}$ & $\omega \sqrt{\beta}\left(\sqrt{\beta} N-Q_{\beta}+\alpha\right)$ & 0 & $2 \sqrt{\beta} \alpha+R(N-1)+2$ \\
\hline$\kappa_{0}$ & \multicolumn{3}{|c|}{$1+\frac{\alpha}{2 N \sqrt{\beta}}$} \\
\hline$\eta_{1}$ & - & $-\sqrt{\beta}\left(\alpha+\sqrt{\beta} N-Q_{\beta}\right)$ & $(1-N)\left(\beta-\frac{R}{2}\right)$ \\
\hline
\end{tabular}


In order to show that the generating function (4.30) satisfies $q$-Virasoro constraints, we have to verify that

$$
\left[\mathrm{T}_{n, i}, \mathcal{S}(\chi)\right]=\text { total difference }=\sum_{\ell \in \mathbb{F}}\left(\mathcal{O}_{\ell}\left(\lambda_{i}+\chi\right)_{i}-\mathcal{O}_{\ell}(\chi)_{i}\right)
$$

for some ( $n$-dependent) operator $\mathcal{O}_{\ell}(\chi)_{i}$ and $\lambda_{i} \in \mathbb{C}$. Indeed, the relation (3.25) can be used to conclude that (4.33) holds true with ${ }^{12}$

$$
\mathcal{O}_{\ell}(X)_{1}=(w)_{2} \mathrm{O}(w)_{1} \otimes \mathrm{S}(w)_{2}, \quad \mathcal{O}_{\ell}(X)_{2}=(w)_{1} \mathrm{~S}(w)_{1} \otimes \mathrm{O}(w)_{2}
$$

Then two commuting sets of $q$-Virasoro constraints for the generating function (4.30) follow by the usual algebra representation (2.31).

\section{Inclusion of Chern-Simons Terms}

In the previous sections we have reviewed how supersymmetric localization allows us to compute partition functions or Wilson loop generating functions of $3 \mathrm{~d} \mathcal{N}=2 \mathrm{U}(N)$ YM-CS theories on various compact spaces. Focusing on theories with no bare CS level, we have shown that such observables have a natural interpretation in terms of what we called the $q$-Virasoro modular double. Exploiting the free boson representation of this construction, we have derived two commuting sets of (infinitely-many) differential constraints that the generating functions have to satisfy.

In this section we analyze the inclusion of a bare CS level and its $q$-Virasoro interpretation. The main observation is that CS terms with integer levels can be represented in the matrix models by "SL(2, $\mathbb{Z})$-squares" of $\Theta$ functions (see comment around (2.45), footnote 7 and [62] for more details)

$$
\mathrm{e}^{-S_{\mathrm{CS}}}=\prod_{j=1}^{N}\left(\frac{\Theta\left(-q_{1}^{1 / 2}\left(w_{j}\right)_{1} ; q_{1}\right)}{\Theta\left(-q_{1}^{1 / 2} ; q_{1}\right)} \frac{\Theta\left(-q_{2}^{1 / 2}\left(w_{j}\right)_{2} ; q_{2}\right)}{\Theta\left(-q_{2}^{1 / 2} ; q_{2}\right)}\right)^{\kappa_{2}}
$$

where $\left(w_{j}\right)_{i}$ is a fundamental weight variable introduced in (4.24) and $\kappa_{2}$ is the integer CS level. In fact, by using the $\operatorname{SL}(2, \mathbb{Z})$ modular properties (A.6), (A.7), (A.8) of the $\Theta$ function and (A.2) we have

$$
\frac{\Theta\left(-q_{1}^{1 / 2}(w)_{1} ; q_{1}\right)}{\Theta\left(-q_{1}^{1 / 2} ; q_{1}\right)} \frac{\Theta\left(-q_{2}^{1 / 2}(w)_{2} ; q_{2}\right)}{\Theta\left(-q_{2}^{1 / 2} ; q_{2}\right)}= \begin{cases}\mathrm{e}^{-\frac{\mathrm{i} \pi X^{2}}{\omega_{1} \omega_{2}}} & : S_{b}^{3} \\ \mathrm{e}^{-\frac{\mathrm{i} \pi X^{2}}{r \omega_{1} \omega_{2}}} \mathrm{e}^{-\frac{\mathrm{i} \pi \ell^{2}}{r}} & : L(r, 1) \\ x^{\ell} & : S^{2} \times S^{1} \text { (index) } \\ x^{\ell} & : S^{2} \times S^{1} \text { (twisted index) }\end{cases}
$$

reproducing the localized CS action on the various backgrounds. From the $q$-Virasoro perspective, the inclusion of CS terms in the gauge theory modifies the external Fock

\footnotetext{
12 For $L(r, 1)$ we require for simplicity that $\sqrt{\beta} \mathrm{P}_{i} / r$ has integer eigenvalues.
} 
states on which we evaluate the free boson operators by inserting additional vertex operators creating particular coherent states ${ }^{13}$

$$
\begin{gathered}
|\alpha\rangle \rightarrow \mathcal{V}_{-}^{\kappa_{2}}|\alpha\rangle, \quad\langle\alpha| \rightarrow\langle\alpha| \mathcal{V}_{+}^{\kappa 2}, \quad \mathcal{V}_{ \pm}=\mathrm{V}_{ \pm, 1} \otimes \mathrm{V}_{ \pm, 2} \\
\mathrm{~V}_{ \pm, i}=\mathrm{e}^{ \pm \sum_{n>0} \frac{(-1)^{n} \lambda_{ \pm n, i}}{\left(q_{i}^{n / 2}-q_{i}^{-n / 2}\right)\left(t_{i}^{n / 2}-t_{i}^{-n / 2}\right)}}
\end{gathered}
$$

where we recall the definition (2.37) of the operators $\lambda_{n, i}$. We can check that the vertex operators (5.3) have the desired property by considering their OPE with the $q$-Virasoro screening currents (we drop a normal ordering constant)

$$
\mathrm{V}_{+, i} \mathrm{~S}(w)_{i} \mathrm{~V}_{-, i}=\mathrm{V}_{-, i} \mathrm{~S}(w)_{i} \mathrm{~V}_{+, i} \Theta\left(-q_{i}^{1 / 2}(w)_{i} ; q_{i}\right)
$$

For instance, in the $q$-Virasoro modular double algebra associated to the $S_{b}^{3}$ geometry studied in Sect. 3 we have

$$
\begin{array}{rl}
\mathcal{V}_{+}^{\kappa_{2}} & \mathcal{Z} \mathcal{V}_{-}^{\kappa_{2}}|\alpha\rangle=\int \mathrm{d}^{N} \underline{X} \mathrm{e}^{-\frac{\mathrm{i} \pi \kappa_{2}}{\omega_{1} \omega_{2}} \sum_{j} X_{j}^{2}} \mathcal{V}_{-}^{\kappa_{2}} \prod_{j} \mathcal{S}\left(X_{j}\right) \mathcal{V}_{+}^{\kappa_{2}}|\alpha\rangle \\
= & \int \mathrm{d}^{N} \underline{X} \Delta_{S}(\underline{X}) \mathrm{e}^{-\frac{\mathrm{i} \pi \kappa_{2}}{\omega_{1} \omega_{2}} \sum_{j} X_{j}^{2}} \mathrm{e}^{\frac{2 \pi \mathrm{i} \omega \sqrt{\beta}}{\omega_{1} \omega_{2}}\left(\sqrt{\beta} N-Q_{\beta}+\alpha\right) \sum_{j} X_{j}} \times \bigotimes_{i=1,2} \\
& \times \exp \left(\sum_{n>0} \frac{\sum_{j}\left(w_{j}^{n}\right)_{i}}{q_{i}^{n / 2}-q_{i}^{-n / 2}} \mathrm{a}_{-n, i}-\sum_{n>0} \frac{\kappa_{2}(-1)^{n}}{\left(q_{i}^{n / 2}-q_{i}^{-n / 2}\right)\left(t_{i}^{n / 2}-t_{i}^{-n / 2}\right)} \lambda_{-n, i}\right) \\
& \mathrm{e}^{\sqrt{\beta} N Q_{i}}|\alpha\rangle,
\end{array}
$$

where we dropped a proportionality factor. Using the algebra representation (2.31) we finally get the matrix model (3.7) with $\kappa_{2} \neq 0$. We also see that while (anti-) fundamental chiral matter can be included by shifting the time variables $\underline{\tau}_{i}$ in the potential $\sum_{j} V\left(X_{j} \mid \underline{\tau}_{1}, \underline{\tau}_{2}\right)$, the inclusion of CS terms shifts the power sums $\sum_{j}^{-}\left(w_{j}^{n}\right)_{i}$. Also, the inclusion of CS terms will modify the differential constraints satisfied by the generating functions, which can be computed by action of the $q$-Virasoro modular double currents on the "dressed" state $\mathcal{V}_{+}^{\kappa_{2}} \mathcal{Z} \mathcal{V}_{-}^{\kappa_{2}}|\alpha\rangle$.

5.1. Decoupling hyper multiplets. Another way to generate an integer CS term in the gauge theory is to couple a pair of fundamental/anti-fundamental chiral multiplets (in fact, a hyper multiplet) and then letting the physical masses go to infinity. In this limit the multiplets can be integrated out and their contribution to the partition function simplifies dramatically leaving behind an integer CS unit. Focusing on the $S_{b}^{3}$ geometry for concreteness, this can be explicitly seen from the 1-loop matter contribution to the matrix model potential

$$
V(X)_{\text {matter }}=-\ln S_{2}(X+M \mid \underline{\omega})-\ln S_{2}(-X+\bar{M} \mid \underline{\omega}),
$$

where $M=-\mathrm{i} M^{\mathbb{R}}+\frac{\omega}{2} \Delta, \bar{M}=-\mathrm{i} \bar{M}^{\mathbb{R}}+\frac{\omega}{2} \bar{\Delta}$ are the complexified masses with $M^{\mathbb{R}}, \bar{M}^{\mathbb{R}}$ and $\Delta, \bar{\Delta}$ being the real masses and Weyl dimensions respectively. We can further

13 The operators $\mathrm{V}_{ \pm, i}$ can be thought of as particular specializations of the vertex operator defined in (2.40) when acting on the (dual) vacuum. While it is possible to use the more general vertex operator (2.40), we use its specializations in order to avoid unnecessary clutterings. 
split $M^{\mathbb{R}}=M_{V}+M_{A}, \bar{M}^{\mathbb{R}}=-M_{V}+M_{A}$, where $M_{V}, M_{A}$ are the vector and axial masses respectively. Upon specializing to $M_{V}=0$ and $\Delta=\bar{\Delta}$, in the decoupling limit $M_{A} \rightarrow \pm \infty$ the matter contribution reduces to

$$
V(X)_{\text {matter }} \sim \operatorname{sign}\left(M_{A}\right) \frac{\mathrm{i} \pi}{\omega_{1} \omega_{2}} X^{2},
$$

where we neglected divergent background terms and used the asymptotic expansion of the double Sine function

$$
\ln S_{2}(X \mid \underline{\omega}) \sim \operatorname{sign}(\operatorname{Im}(X)) \frac{\mathrm{i} \pi}{2 \omega_{1} \omega_{2}}\left(X^{2}-\omega X+\frac{\omega^{2}+\omega_{1} \omega_{2}}{6}\right), \quad|X| \rightarrow \infty .
$$

We can now recognize in the above contribution an induced CS level $\kappa_{2}=-\operatorname{sign}\left(M_{A}\right)$.

From the $q$-Virasoro viewpoint, the coupling/decoupling procedure of the chiral multiplet pair involves the insertion of the modular double version of the vertex operator (2.36), which for the $S_{b}^{3}$ geometry reads as

$$
\mathcal{H}_{\gamma}(Z)=\mathrm{H}_{\gamma}\left(\mathrm{e}^{\frac{2 \pi \mathrm{i}}{\omega_{1}} Z}\right)_{1} \otimes \mathrm{H}_{\gamma}\left(\mathrm{e}^{\frac{2 \pi \mathrm{i}}{\omega_{2}} Z}\right)_{2}
$$

and the large momentum limit $\operatorname{Im}(\omega \beta \gamma) \rightarrow \pm \infty$. In order to see that we can take its OPE with the modular double screening current, yielding

$$
\mathcal{H}_{\gamma}(Z) \mathcal{S}(X)=: \mathcal{H}_{\gamma}(Z) \mathcal{S}(X): \frac{\mathrm{e}^{\frac{\mathrm{i} \pi}{\omega_{1} \omega_{2}} \gamma \beta \omega(X+Z)}}{S_{2}\left(\frac{\omega}{2}+\frac{\gamma \beta \omega}{2}+X-Z \mid \underline{\omega}\right) S_{2}\left(\frac{\omega}{2}+\frac{\gamma \beta \omega}{2}-X+Z \mid \underline{\omega}\right)} .
$$

When acting on the charged Fock vacuum $|\alpha\rangle$ we obtain

$$
\begin{aligned}
& \mathcal{H}_{\gamma}(Z) \mathcal{S}(X)|\alpha\rangle=\frac{\mathrm{e}^{\frac{\pi \mathrm{i} \omega}{\omega_{1} \omega_{2}} Z(\beta \gamma+\sqrt{\beta} \gamma \alpha)} \mathrm{e}^{\frac{2 \pi \mathrm{i} \omega}{\omega_{1} \omega_{2}} X\left(1+\sqrt{\beta} \alpha+\frac{\beta \gamma}{2}\right)}}{S_{2}\left(\frac{\omega}{2}+\frac{\gamma \beta \omega}{2}+X-Z \mid \underline{\omega}\right) S_{2}\left(\frac{\omega}{2}+\frac{\gamma \beta \omega}{2}-X+Z \mid \underline{\omega}\right)} \\
& \quad \times \bigotimes_{i=1,2} \exp \left(\sum_{n>0} \frac{\mathrm{e}^{\frac{2 \pi \mathrm{i} n}{\omega_{i}} X}}{q_{i}^{n / 2}-q_{i}^{-n / 2}} \mathrm{a}_{-n, i}+\sum_{n>0} \frac{\left(t_{i}^{\gamma / 2}-t_{i}^{-\gamma n / 2}\right) \mathrm{e}^{\frac{2 \pi \mathrm{i} n}{\omega_{i}} Z}}{\left(q_{i}^{n / 2}-q_{i}^{-n / 2}\right)\left(t_{i}^{n / 2}-t_{i}^{-n / 2}\right)} \lambda_{-n, i}\right) \\
& \mathrm{e}^{\sqrt{\beta} \mathrm{Q}_{i}\left(1+\frac{\gamma}{2}\right)}|\alpha\rangle,
\end{aligned}
$$

and upon shifting $\alpha \rightarrow \alpha-\sqrt{\beta} \gamma / 2$ and taking the $\operatorname{limit} \operatorname{Im}(\omega \beta \gamma) \rightarrow \pm \infty$ we get an effective contribution $\kappa_{2}=\operatorname{sign}(\operatorname{Im}(\omega \beta \gamma))$ to the CS level in the matrix model potential.

It is worth observing that this mechanism is essentially equivalent to the one we have discussed previously: in fact, on the one hand we can write

$$
\mathcal{H}_{\gamma}(Z) \mathcal{S}(X)=\left[\mathcal{H}_{\gamma}(Z)\right]_{0}\left[\mathcal{H}_{\gamma}(Z)\right]_{-} \mathcal{S}(X)\left[\mathcal{H}_{\gamma}(Z)\right]_{+} \operatorname{OPE}(X),
$$

where []$_{ \pm, 0}$ denotes the positive, negative or zero mode part and $\operatorname{OPE}(X)$ is the normal ordering function giving rise to the CS level in the limit; on the other hand we can split $\left[\mathcal{H}_{\gamma}(Z)\right]_{ \pm}=\left[\mathcal{V}_{\gamma}(Z) \mathcal{V}_{-\gamma}(Z)^{-1}\right]_{ \pm}$as in (2.41), and in the limit one of the component 
vertices is predominant over the other and the resulting action on the vacuum is essentially equivalent to the action of $\mathcal{V}_{ \pm}$defined in (5.3).

5.2. Pure Chern-Simons and torus knots. Since through the inclusion of the vertex operators (5.3) we can introduce CS terms in our $q$-Virasoro matrix models, it is interesting to investigate the relation of the latter to pure CS matrix models [120,121]. Focusing again on the $S_{b}^{3}$ geometry, we immediately see that upon setting $\beta=1 / 2$ the generating function (3.7) reduces to

$$
\begin{aligned}
Z\left(\underline{\tau}_{1}, \underline{\tau}_{2}\right)= & (-4)^{\frac{N(N-1)}{2}} \int_{\mathrm{i} \mathbb{R}^{N}} \mathrm{~d}^{N} \underline{X} \prod_{k<j} \sin \left(\pi \frac{X_{k}-X_{j}}{\omega_{1}}\right) \sin \left(\pi \frac{X_{k}-X_{j}}{\omega_{2}}\right) \\
& \times \mathrm{e}^{\frac{\mathrm{i} \pi \kappa_{2}}{\omega_{1} \omega_{2}} \sum_{j} X_{j}^{2}} \mathrm{e}^{\frac{2 \pi \mathrm{i} \kappa_{1}}{\omega_{1} \omega_{2}} \sum_{j} X_{j}} \prod_{i=1,2} \exp \left(\sum_{n>0} \tau_{n, i} \sum_{j} \mathrm{e}^{\frac{2 \pi \mathrm{i} n}{\omega_{i}} X_{j}}+\tau_{0, i} N \kappa_{0}\right),
\end{aligned}
$$

corresponding to the generating function of Wilson loops in pure CS theory on $S_{b}^{3}$. Physically, the value $\beta=1 / 2$ corresponds to a massless adjoint chiral multiplet, and hence its 1-loop contribution is trivial due to cancellations between opposite roots.

In contrast to Sect. 3.3, where we considered $\beta=1 / 2$ as a particular case in the round $S^{3}$ geometry, here the algebra of the constraints is still given by the $q$-Virasoro modular double.

A particularly interesting situation is when $\omega_{1}$ and $\omega_{2}$ are two coprime integers. In this case the matrix model (5.13) corresponds to the Wilson loop generating function for torus knots. Notice that in this limit the deformation parameters $q_{1}=\mathrm{e}^{2 \pi \mathrm{i} \frac{\omega}{\omega_{1}}}$ and $q_{2}=\mathrm{e}^{2 \pi \mathrm{i} \frac{\omega}{\omega_{2}}}$ both go to roots of unity. It is also known that the matrix integral (5.13) satisfy usual Virasoro constraints in this limit [122].

5.3. Refined Chern-Simons. The $q$-Virasoro matrix model (2.32), or equivalently the $D^{2} \times S^{1}$ generating function (2.51), is of refined CS type [2-4]. In fact, the vector and adjoint multiplets provide the Macdonald integration measure $\Delta_{\beta}(\underline{w} ; q)(2.27)$. Considering an $S^{1}$ fibration over $S^{2}$ with first Chern class $\kappa$, the partition function of refined CS theory reads as

$$
Z_{\mathrm{rCS}}(\kappa)=\int \mathrm{d}^{N} \underline{W} \Delta_{\beta_{\mathrm{rCS}}}\left(\mathrm{e}^{\underline{W}} ; q_{\mathrm{rCS}}\right) \mathrm{e}^{-\frac{\kappa}{2 g_{s}} \sum_{j} W_{j}^{2}},
$$

where we used the parametrization $w=\mathrm{e}^{W}$ and $q_{\mathrm{rCS}}=\mathrm{e}^{g_{s}}$. In particular, $\kappa=0,1$ corresponds to $S^{2} \times S^{1}$ and $S^{3}$ respectively. Given the relation between the $q$-Virasoro matrix model and refined CS, it is natural to ask whether there is any relation between $q$-Virasoro modular double matrix models, or equivalently $3 \mathrm{~d}$ compact space generating functions, and refined CS. For instance, the $S_{b}^{3}$ partition function (3.7) with $\kappa_{1}=0$ reads as

$$
Z(\underline{0}, \underline{0})=\int_{\mathbb{i}^{N}} \mathrm{~d}^{N} \underline{X} \Delta_{S}(\underline{X}) \mathrm{e}^{-\frac{\mathrm{i} \pi \kappa_{2}}{\omega_{1} \omega_{2}} \sum_{j} X_{j}^{2}} .
$$

In order to establish a clear relation with refined CS, let us start by taking the specialization $\beta \omega=b_{2} \omega_{2}, b_{2} \in \mathbb{Z}_{>0}$, in the $S_{b}^{3}$ matrix model, in which case the measure simplifies to 


$$
\begin{aligned}
\left.\Delta_{S}(\underline{X})\right|_{\beta=\frac{b_{2} \omega_{2}}{\omega}}=2^{b_{2} N(N-1)} \prod_{j \neq k} \prod_{n=0}^{b_{2}-1} \sin \left(\pi \frac{n \omega_{2}+X_{j}-X_{k}}{\omega_{1}}\right) \\
\left.\quad=\left(-2 \mathrm{i}^{b_{2}}\right)^{b_{2} N(N-1)} \prod_{j \neq k}^{b_{2}-1} \prod_{n=0}^{\frac{\mathrm{i} \pi}{\omega_{1}}\left(n \omega_{2}+X_{j}-X_{k}\right)}-\mathrm{e}^{-\frac{\mathrm{i} \pi}{\omega_{1}}\left(n \omega_{2}+X_{j}-X_{k}\right)}\right) .
\end{aligned}
$$

On the other hand, the Macdonald measure for $\beta_{\mathrm{rCS}} \in \mathbb{Z}_{>0}$ (which is a common specialization in refined CS) simplifies to

$$
\begin{aligned}
& \left.\Delta_{\beta_{\mathrm{rCS}}}\left(\mathrm{e}^{\frac{W}{-}} ; q_{\mathrm{rCS}}\right)\right|_{\beta_{\mathrm{rCS}} \in \mathbb{Z}_{>0}}=\prod_{k \neq j} \prod_{n=0}^{\beta_{\mathrm{rCS}}-1}\left(1-q_{\mathrm{rCS}}^{n} \mathrm{e}^{W_{j}-W_{k}}\right) \\
& =q_{\mathrm{rCS}}^{N(N-1) \frac{\beta_{\mathrm{rCS}}\left(\beta_{\mathrm{rCS}}-1\right)}{4}} \prod_{j \neq k} \prod_{n=0}^{\beta_{\mathrm{rCS}}-1}\left(q_{\mathrm{rCS}}^{\frac{n}{2}} \mathrm{e}^{\frac{W_{j}-W_{k}}{2}}-q_{\mathrm{rCS}}^{-\frac{n}{2}} \mathrm{e}^{\frac{W_{k}-W_{j}}{2}}\right),
\end{aligned}
$$

implying that the $S_{b}^{3}$ matrix model collapses to the refined CS matrix model upon identifying $g_{s}=2 \pi \mathrm{i} \omega_{2} / \omega_{1}, \beta_{\mathrm{rCS}}=b_{2}, W=2 \pi \mathrm{i} X / \omega_{1}$ and $\kappa=\kappa_{2}$. Similarly, if we take the more general specialization $\beta \omega=b_{1} \omega_{1}+b_{2} \omega_{2}, b_{1,2} \in \mathbb{Z}_{>0}$, the $\Delta_{S}(\underline{X})$ measure collapses to two copies of the Macdonald measure due to (A.11). We can in fact relax any specialization of the parameters and consider instead the limit where the $S_{b}^{3}$ is very squashed, i.e. $\left|\omega_{1} / \omega_{2}\right| \gg 1$, in which case the double Sine function has the semiclassical behaviour (assuming $\operatorname{Im}\left(\omega_{2} / \omega_{1}\right)>0$ )

$$
S_{2}(X \mid \underline{\omega})=\mathrm{e}^{\mathrm{i} \pi} \frac{\mathrm{i}}{2} B_{22}(X \mid \underline{\omega})\left(\mathrm{e}^{\frac{2 \pi \mathrm{i}}{\omega_{1}} X} ; \mathrm{e}^{2 \pi \mathrm{i} \frac{\omega_{2}}{\omega_{1}}}\right)_{\infty}\left(1+O\left(\mathrm{e}^{2 \pi \mathrm{i} \frac{\omega_{1}}{\omega_{2}}}\right)\right),
$$

and hence

$$
\Delta_{S}(\underline{X})=\Delta_{\beta}\left(\mathrm{e}^{\frac{2 \pi \mathrm{i}}{\omega_{1}} \underline{X}} ; \mathrm{e}^{2 \pi \mathrm{i} \frac{\omega_{2}}{\omega_{1}}}\right)\left(1+O\left(\mathrm{e}^{2 \pi \mathrm{i} \frac{\omega_{1}}{\omega_{2}}}\right)\right),
$$

up to proportionality factors. Therefore, the $q$-Virasoro modular double might give rise to a doubled or non-perturbative version of refined CS. Moreover, it is known that the (large $N$ limit of) refined CS observables are captured by refined (closed) open topological strings $[2,4,123]$, and thus one can expect that the $q$-Virasoro modular double might also play a role in the non-perturbative description of refined topological strings. This observation is in line with those of $[61,97]$, and we will comment more on this aspect in Sect. 7.

\section{Generalization to Quiver Gauge Theories}

Our discussion on the $q$-Virasoro structures in $3 \mathrm{~d} \mathcal{N}=2$ YM-CS theories has so far focused on a single node $\mathrm{U}(N)$ gauge group coupled to 1 adjoint and possibly (anti-) fundamental chirals. The goal of this section is to show that our construction admits a generalization to a huge class of $3 \mathrm{~d} \mathcal{N}=2$ unitary quiver gauge theories and $\mathrm{W}_{q, t}(\Gamma)$ algebras of [73].

Let us start by recalling some algebraic definition from [73]. A quiver $\Gamma$ is a collection of nodes $\Gamma_{0}$ and arrows $\Gamma_{1}$, see Fig. 1 for an example. Given two nodes $a, b \in \Gamma_{0}$ and an arrow $\Gamma_{1} \ni e: a \rightarrow b$, we can associate to the quiver the deformed Cartan matrix $C_{a b} \in\left|\Gamma_{0}\right| \times\left|\Gamma_{0}\right|$

$$
C_{a b}=\left(1+p^{-1}\right) \delta_{a b}-\sum_{e: b \rightarrow a} m_{e}^{-1}-p^{-1} \sum_{e: a \rightarrow b} m_{e},
$$




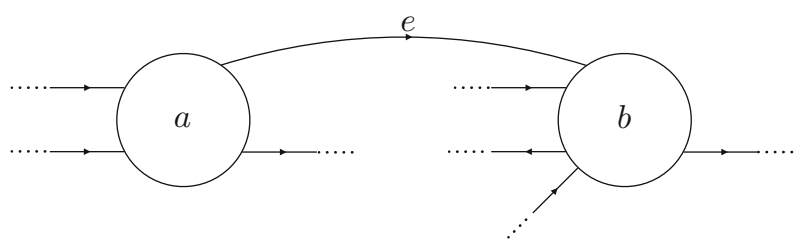

Fig. 1. Portion of a quiver $\Gamma$. We explicitly displayed 2 nodes $a, b \in \Gamma_{0}$, an arrow $e \in \Gamma_{1}$ from $a$ to $b$, and several arrows with source or target in $a$ or $b$

the Heisenberg algebra (we display non-vanishing commutators only)

$\left[\mathrm{a}_{n}^{a}, \mathrm{a}_{m}^{b}\right]=\frac{1}{n}\left(q^{\frac{n}{2}}-q^{-\frac{n}{2}}\right)\left(t^{\frac{n}{2}}-t^{-\frac{n}{2}}\right) p^{\frac{n}{2}} C_{a b}^{[n]} \delta_{n+m, 0}, \quad\left[\mathrm{P}^{a}, \mathrm{Q}^{b}\right]=C_{a b}^{[0]}, n, m \in \mathbb{Z} \backslash\{0\}$,

and the screening current

$$
\mathrm{S}^{a}(w)=: \mathrm{e}^{-\sum_{n \neq 0} \frac{w^{-n}}{q^{n / 2}-q^{-n / 2}} \mathbf{a}_{n}^{a}}: \mathrm{e}^{\sqrt{\beta} \mathrm{Q}^{a}} w^{\sqrt{\beta} \mathrm{P}^{a}},
$$

where $q, t, p=q t^{-1}, m_{e} \in \mathbb{C}^{14}$, while the ${ }^{[n]}$ operation means replacing each parameter with its $n^{\text {th }}$ power, for instance

$$
C_{a b}^{[n]}=\left(1+p^{-n}\right) \delta_{a b}-\sum_{e: b \rightarrow a} m_{e}^{-n}-p^{-n} \sum_{e: a \rightarrow b} m_{e}^{n} .
$$

With these data the $\mathrm{W}_{q, t}(\Gamma)$ algebra can be defined to be the non-commutative associative algebra generated by the currents $\left\{\mathrm{T}^{a}(z)=\sum_{n \in \mathbb{Z}} \mathrm{T}_{n}^{a} z^{-n}, a \in \Gamma_{0}\right\}$ and given as the commutant up to total differences of the screening currents in the Heisenberg algebra

$$
\left[\mathrm{T}_{n}^{a}, \mathrm{~S}^{b}(w)\right]=\text { total difference. }
$$

For instance, the single node quiver $\Gamma_{0}=\{1\}, \Gamma_{1}=\{\emptyset\}$ corresponding to the $A_{1}$ Lie algebra diagram gives rise to the $q$-Virasoro $=\mathrm{W}_{q, t}\left(A_{1}\right)$ algebra reviewed in Sect. 2, whereas the $n$-node quiver $\Gamma_{0}=\{1, \ldots, n\}, \Gamma_{1}=\left\{e_{a}: a \rightarrow a+1, a=1, \ldots, n-1\right\}$ corresponding to the $A_{n}$ Lie algebra gives rise to the $\mathrm{W}_{q, t}\left(A_{n}\right)$ algebra of [124]. More generally, for quivers associated to simple Lie algebras the construction of [73] agrees with [114].

Following the discussion of Sect. 2.3, we can now associate to the $\mathrm{W}_{q, t}(\Gamma)$ algebra a $3 \mathrm{~d} \mathcal{N}=2$ unitary quiver gauge theory on $D^{2} \times S^{1}$, whose Wilson loop generating function will be reproduced by the action of the $\mathrm{W}_{q, t}(\Gamma)$ screening charges on a charged Fock vacuum $|\underline{\alpha}\rangle, \underline{\alpha}=\left\{\alpha_{a}, a \in \Gamma_{0}\right\}$, namely

$$
Z\left(\left\{\underline{\tau}^{a}\right\}\right) \simeq \oint \prod_{a=1}^{\left|\Gamma_{0}\right|} \frac{\mathrm{d}^{N_{a}} \underline{w}_{a}}{2 \pi \underline{\mathrm{i}}_{a}} \prod_{a=1}^{\left|\Gamma_{0}\right|} \prod_{j=1}^{N_{a}} \mathrm{~S}^{a}\left(w_{a, j}\right)|\underline{\alpha}\rangle .
$$

It is important at this point to not confuse the $\Gamma$ quiver of the algebra with the unitary quiver of the $3 \mathrm{~d}$ theory. For instance, the $A_{1}$ quiver associated to the $q$-Virasoro algebra has a single node and no arrows at all, while the dual gauge theory involves a $\mathrm{U}(N)$ gauge vector and 1 adjoint chiral multiplet, whose quiver description usually consists of

14 To compare with [73] we have to set $(q)_{\text {here }}=\left(q_{2}\right)_{\text {there }},(t)_{\text {here }}=\left(q_{1}^{-1}\right)_{\text {there }}$ 
$A_{1}$ quiver

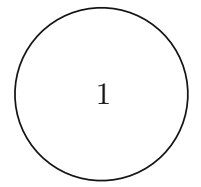

3 d quiver

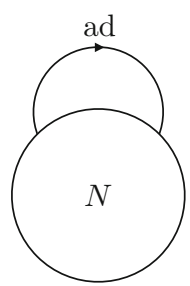

Fig. 2. The quivers of $q$-Virasoro (left) and the corresponding $3 \mathrm{~d}$ gauge theory (right)

a round node for the gauge group and a loop arrow for the adjoint, as depicted in Fig. 2. In order to determine the dual $3 \mathrm{~d}$ gauge theory description for the general case, the key point is to understand the measure arising from the product of several screening currents

$$
\begin{aligned}
& \prod_{a=1}^{\left|\Gamma_{0}\right|} \prod_{j=1}^{N_{a}} \mathrm{~S}^{a}\left(w_{a, j}\right)=: \prod_{a=1}^{\left|\Gamma_{0}\right|} \prod_{j=1}^{N_{a}} \mathrm{~S}^{a}\left(w_{a, j}\right): \prod_{a=1}^{\left|\Gamma_{0}\right|} c_{\beta}\left(w_{a}, 1 ; q\right) \Delta_{\beta}\left(w_{a} ; q\right) \prod_{j=1}^{N_{a}} w_{a, j}^{\beta\left(N_{a}-1\right)} \\
& \quad \times \prod_{a=1}^{\left|\Gamma_{0}\right|} \prod_{e: a \rightarrow a} \frac{1}{c_{\beta}\left(w_{a}, m_{e} ; q\right)} \prod_{1 \leq j \neq k \leq N_{a}} \frac{\left(t m_{e} w_{a, k} w_{a, j}^{-1} ; q\right)_{\infty}}{\left(m_{e} w_{a, k} w_{a, j}^{-1} ; q\right)_{\infty}} \prod_{j=1}^{N_{a}} w_{a, j}^{-\beta\left(N_{a}-1\right)} \\
& \quad \times \prod_{1 \leq a<b \leq\left|\Gamma_{0}\right|} \prod_{j=1}^{N_{a}} \prod_{k=1}^{N_{b}} \prod_{e: a \rightarrow b} \frac{\left(t m_{e} w_{b, k} w_{a, j}^{-1} ; q\right)_{\infty}}{\left(m_{e} w_{b, k} w_{a, j}^{-1} ; q\right)_{\infty}} w_{a, j}^{-\beta} \\
& \quad \times \prod_{e: b \rightarrow a} \frac{\left(q m_{e}^{-1} w_{b, k} w_{a, j}^{-1} ; q\right)_{\infty}}{\left(q t^{-1} m_{e}^{-1} w_{b, k} w_{a, j}^{-1} ; q\right)_{\infty}} w_{a, j}^{-\beta} .
\end{aligned}
$$

From this expression we can immediately read off the corresponding $3 \mathrm{~d} \mathcal{N}=2$ gauge theory: it is a $\Gamma$ quiver YM theory with $\mathrm{U}\left(N_{a}\right)$ gauge nodes each coupled to 1 adjoint chiral multiplet, 1 bi-fundamental hyper multiplet (actually a pair of fundamental/antifundamental chirals) for each arrow connecting different gauge nodes and 1 adjoint hyper multiplet (a pair of adjoint chirals) for each loop edge. The generating function of the theory is identified with a highest weight state of the $\mathrm{W}_{q, t}(\Gamma)$ algebra and will satisfy the associated constraints

$$
T^{a}\left(z \mid \underline{\tau}^{a}\right) Z\left(\left\{\underline{\tau}^{a}\right\}\right)=\operatorname{Pol}(z) \Rightarrow T_{n}^{a}\left(\underline{\tau}^{a}\right) Z\left(\left\{\underline{\tau}^{a}\right\}\right)=0, \quad n>0
$$

by construction. (Anti-)fundamental chiral multiplets or CS levels for each gauge node can be added on top of this construction as insertion of addition vertex operators, and may be represented by auxiliary square nodes or integer labels respectively.

As it should be clear from the previous sections, if we want to discuss $3 \mathrm{~d}$ theories on compact spaces we should construct the modular double of the $\mathrm{W}_{q, t}(\Gamma)$ algebras. In the $q$-Virasoro $=\mathrm{W}_{q, t}\left(A_{1}\right)$ case, our construction of the modular double only relied on the property (6.5) of the screening currents, and therefore we can generalize our analysis to arbitrary $\mathrm{W}_{q, t}(\Gamma)$ algebras following the recipe given in Sects. 3, 4 for the various geometries. As an application, in the following we will consider the simple but important example of the (mass deformed) $\mathrm{ABJ}(\mathrm{M})$ theory on $S_{b}^{3}$, where the relevant quivers are shown in Fig. 3. 
$\Gamma$ quiver

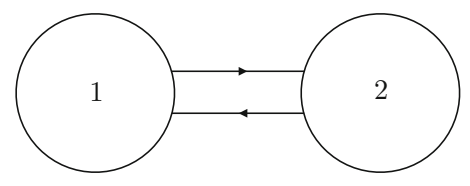

3d quiver

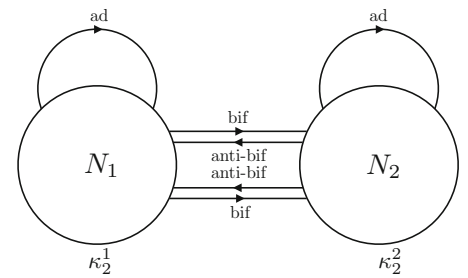

Fig. 3. The "ABJ(M) quiver" of the algebra (left) and the gauge theory (right)

6.1. $A B J(M)$ theory. The ABJ theory [10] is the $\mathcal{N}=6 \mathrm{U}\left(N_{1}\right)_{\kappa_{2}} \times \mathrm{U}\left(N_{2}\right)_{-\kappa_{2}} \mathrm{CS}$ theory with 1 bi-fundamental and 1 anti-bi-fundamental hyper multiplets, where the subindex denotes the CS level. In the case $N_{1}=N_{2}$ the theory specializes to the ABJM model [9]. Using the notation of Sect. 3, its $S_{b}^{3}$ partition function reads as (see for instance [125])

$$
\begin{aligned}
Z_{\mathrm{ABJ}}= & \mathcal{N}_{0} \int_{\mathrm{i} \mathbb{R}} \prod_{a=1,2} \mathrm{~d}^{N_{a}} \underline{X}_{a} \mathrm{e}^{-\frac{\mathrm{i} \pi \kappa_{2}}{\omega_{1} \omega_{2}}\left(\sum_{j=1}^{N_{1}} X_{1, j}^{2}-\sum_{k=1}^{N_{2}} X_{2, k}^{2}\right)} \\
& \times \prod_{a=1,2} \prod_{1 \leq j<k \leq N_{a}}(2 \mathrm{i})^{2} \sin \left(\pi \frac{X_{a, j}-X_{a, k}}{\omega_{1}}\right) \sin \left(\pi \frac{X_{a, j}-X_{a, k}}{\omega_{2}}\right) \\
& \times \prod_{j=1}^{N_{1}} \prod_{k=1}^{N_{2}} \frac{S_{2}\left(\frac{\omega}{2}+X_{2, k}-X_{1, j}+\frac{\omega}{4} \mid \underline{\omega}\right)^{2}}{S_{2}\left(\frac{\omega}{2}+X_{2, k}-X_{1, j}-\frac{\omega}{4} \mid \underline{\omega}\right)^{2}} .
\end{aligned}
$$

In order to describe the partition function (or the generating function) of the ABJ theory through $\mathrm{W}_{q, t}(\Gamma)$ techniques, let us start by considering a two node quiver $\Gamma$ with two oppositely oriented ${ }^{15}$ arrows connecting the two nodes as in Fig. 3. The corresponding product of screening currents is a simple specialization of (6.7)

$$
\begin{aligned}
\prod_{a=1}^{2} \prod_{j=1}^{N_{a}} \mathrm{~S}^{a}\left(w_{a, j}\right)= & : \prod_{a=1}^{2} \prod_{j=1}^{N_{a}} \mathrm{~S}^{a}\left(w_{a, j}\right): \Delta_{\beta}\left(\underline{w}_{a} ; q\right) c_{\beta}\left(\underline{w}_{a} ; q\right) \prod_{a=1}^{2} \prod_{j=1}^{N_{a}} w_{a, j}^{\beta\left(N_{a}-1\right)} \\
& \times \prod_{j=1}^{N_{1}} w_{1, j}^{-2 \beta N_{2}} \prod_{k=1}^{N_{2}} \frac{\left(t m_{12} w_{2, k} w_{1, j}^{-1} ; q\right)_{\infty}}{\left(m_{12} w_{2, k} w_{1, j}^{-1} ; q\right)_{\infty}} \frac{\left(q m_{21}^{-1} w_{2, k} w_{1, j}^{-1} ; q\right)_{\infty}}{\left(q t^{-1} m_{21}^{-1} w_{2, k} w_{1, j}^{-1} ; q\right)_{\infty}}
\end{aligned}
$$

where we set $m_{e}=m_{12}$ for the arrow $e: 1 \rightarrow 2$ and $m_{e}=m_{21}$ for the arrow $e: 2 \rightarrow 1$. In order to describe the theory on $S_{b}^{3}$ we consider the modular double construction of Sect. 3, namely we define

$$
\begin{aligned}
\mathcal{S}^{a}\left(X_{a, j}\right) & =\left(w_{a, j}\right)_{1}\left(w_{a, j}\right)_{2} \mathrm{~S}^{a}\left(w_{a, j}\right)_{1} \otimes \mathrm{S}^{a}\left(w_{a, j}\right)_{2}, \\
\left(w_{a, j}\right)_{i} & =\mathrm{e}^{\frac{2 \pi \mathrm{i}}{\omega_{i}} X_{a, j}}, \quad\left(m_{e}\right)_{i}=\mathrm{e}^{\frac{2 \pi \mathrm{i}}{\omega_{i}} M_{e}}, \quad e \in\{(12),(21)\}, \quad i=1,2,
\end{aligned}
$$

with the $\operatorname{SL}(2, \mathbb{Z})$ gluing as in table (3.13). Here $M_{e}$ are interpreted as the complexified masses for the bi-fundamental hypers, namely $i M_{e}=M_{e}^{\mathbb{R}}+\mathrm{i} \frac{\omega}{2} \Delta$ where $\Delta$ is the Weyl

15 The orientation does not actually matter for the $\mathrm{ABJ}(\mathrm{M})$ theory. 
dimension, which we take to be $\Delta=1 / 2$. The corresponding product of screening charges yields the operator

$$
\begin{aligned}
\mathcal{Z}= & \int_{\mathrm{i} \mathbb{R}} \prod_{a=1,2} \mathrm{~d}^{N_{a}} \underline{X}_{a} \prod_{a=1}^{2} \prod_{j=1}^{N_{a}} \mathcal{S}^{a}\left(X_{a, j}\right) \\
= & \mathrm{e}^{-\frac{\mathrm{i} \pi \omega \beta N_{1} N_{2}}{\omega_{1} \omega_{2}}\left(M_{12}-M_{21}\right)} \int_{i \mathbb{R}} \prod_{a=1,2} \mathrm{~d}^{N_{a}} \underline{X}_{a}: \prod_{a=1}^{2} \prod_{j=1}^{N_{a}} \mathcal{S}^{a}\left(X_{a, j}\right): \\
& \times \prod_{a=1,2} \mathrm{e}^{\frac{2 \pi \mathrm{i} \omega \sqrt{\beta}}{\omega_{1} \omega_{2}}\left((-1)^{a} \sqrt{\beta}\left(N_{2}-N_{1}\right)-Q_{\beta}\right) \sum_{j=1}^{N_{a}} X_{a, j}} \Delta_{S}\left(\underline{X}_{a}\right) \\
& \times \prod_{j=1}^{N_{1}} \prod_{k=1}^{N_{2}} \frac{S_{2}\left(\omega-M_{21}+X_{2, k}-X_{1, j} \mid \underline{\omega}\right) S_{2}\left(\beta \omega+M_{12}+X_{2, k}-X_{1, j} \mid \underline{\omega}\right)}{S_{2}\left(\omega-\omega \beta-M_{21}+X_{2, k}-X_{1, j} \mid \underline{\omega}\right) S_{2}\left(M_{12}+X_{2, k}-X_{1, j} \mid \underline{\omega}\right)} .
\end{aligned}
$$

We now include CS terms as discussed in Sect. 5. The $\mathrm{W}_{q, t}(\Gamma)$ generalization of the vertex operators (5.3) is given by

$$
\mathcal{V}_{ \pm}^{a}=\mathrm{V}_{ \pm, 1}^{a} \otimes \mathrm{V}_{ \pm, 2}^{a}, \quad \mathrm{~V}_{ \pm, i}^{a}=\mathrm{e}^{ \pm \sum_{n>0} \frac{(-1)^{n} \lambda_{ \pm n, i}^{a}}{\left(q_{i}^{n / 2}-q_{i}^{-n / 2}\right)\left(t_{i}^{n / 2}-t_{i}^{-n / 2}\right)}}
$$

where we have introduced the basis

$$
\lambda_{n, i}^{a}=\mathrm{a}_{n, i}^{b}\left(C_{i}^{[-n]}\right)_{b a}^{-1} p_{i}^{\frac{n}{2}}, \quad \mathrm{P}_{\lambda, i}^{a}=\mathrm{P}_{i}^{b}\left(C_{i}^{[0]}\right)_{b a}^{-1}, \quad \mathrm{Q}_{\lambda, i}^{a}=\mathrm{Q}_{i}^{b}\left(C_{i}^{[0]}\right)_{b a}^{-1}, \quad n \in \mathbb{Z} \backslash\{0\}
$$

of the two commuting $(i=1,2)$ Heisenberg algebras satisfying (we display non-trivial relations only)

$$
\begin{gathered}
{\left[\mathrm{a}_{n, i}^{a}, \lambda_{m, i}^{b}\right]=\frac{1}{n}\left(q_{i}^{\frac{n}{2}}-q_{i}^{-\frac{n}{2}}\right)\left(t_{i}^{\frac{n}{2}}-t_{i}^{-\frac{n}{2}}\right) \delta_{a, b} \delta_{n+m, 0},} \\
{\left[\mathrm{P}_{i}^{a}, \mathrm{Q}_{\lambda, i}^{b}\right]=\left[\mathrm{P}_{\lambda, i}^{a}, \mathrm{Q}_{i}^{b}\right]=\delta_{a, b}, \quad n, m \in \mathbb{Z} \backslash\{0\} .}
\end{gathered}
$$

We can now consider the dressed operator

$$
\begin{aligned}
& \prod_{a=1,2}\left(\mathcal{V}_{+}^{a}\right)^{\kappa_{2}^{a}} \mathcal{Z} \prod_{a=1,2}\left(\mathcal{V}_{-}^{a}\right)^{\kappa_{2}^{a}} \\
& =\mathrm{e}^{-\frac{\mathrm{i} \pi \omega \beta N_{1} N_{2}}{\omega_{1} \omega_{2}}\left(M_{12}-M_{21}\right)} \int_{\mathrm{i} \mathbb{R}} \prod_{a=1,2} \mathrm{~d}^{N_{a}} \underline{X}_{a}: \prod_{a=1}^{2}\left(\mathcal{V}_{+}^{a}\right)^{\kappa_{2}^{a}}\left(\prod_{j=1}^{N_{a}} \mathcal{S}^{a}\left(X_{a, j}\right)\right)\left(\mathcal{V}_{-}^{a}\right)^{\kappa_{2}^{a}}: \\
& \quad \times \prod_{a=1,2} \mathrm{e}^{-\frac{\mathrm{i} \pi \kappa_{2}^{a}}{\omega_{1} \omega_{2}} \sum_{j=1}^{N_{a}} X_{a, j}^{2} \mathrm{e}^{\frac{2 \pi \mathrm{i} \omega \sqrt{\beta}}{\omega_{1} \omega_{2}}\left((-1)^{a} \sqrt{\beta}\left(N_{2}-N_{1}\right)-Q_{\beta}\right) \sum_{j=1}^{N_{a}} X_{a, j}} \Delta_{S}\left(\underline{X}_{a}\right)} \\
& \quad \times \prod_{j=1}^{N_{1}} \prod_{k=1}^{N_{2}} \frac{S_{2}\left(\omega-M_{21}+X_{2, k}-X_{1, j} \mid \underline{\omega}\right) S_{2}\left(\beta \omega+M_{12}+X_{2, k}-X_{1, j} \mid \underline{\omega}\right)}{S_{2}\left(\omega-\omega \beta-M_{21}+X_{2, k}-X_{1, j} \mid \underline{\omega}\right) S_{2}\left(M_{12}+X_{2, k}-X_{1, j} \mid \underline{\omega}\right)}
\end{aligned}
$$

where the equality holds up to constant proportionality factors. The action of this operator on the charged Fock vacuum $|\underline{\alpha}\rangle$ defined by 


$$
\underline{\alpha}=\left\{\alpha_{a}, a=1,2\right\}, \quad|\underline{\alpha}\rangle=\otimes_{i=1,2} \mathrm{e}^{\sum_{a} \alpha_{a} \mathrm{Q}_{\lambda, i}^{a}}|0\rangle, \quad \mathrm{a}_{n>0}^{a}|0\rangle=0, \quad \mathrm{P}_{i}^{a}|\underline{\alpha}\rangle=\alpha_{a}|\underline{\alpha}\rangle,
$$

yields the state

$$
\begin{aligned}
& \prod_{a=1,2}\left(\mathcal{V}_{+}^{a}\right)^{\kappa_{2}^{a}} \mathcal{Z} \prod_{a=1,2}\left(\mathcal{V}_{-}^{a}\right)^{\kappa_{2}^{a}}|\underline{\alpha}\rangle \\
& =\mathrm{e}^{-\frac{\mathrm{i} \pi \omega \beta N_{1} N_{2}}{\omega_{1} \omega_{2}}\left(M_{12}-M_{21}\right)} \int_{\mathrm{i} \mathbb{R}} \prod_{a=1,2} \mathrm{~d}^{N_{a}} \underline{X}_{a} \prod_{a=1,2} \mathrm{e}^{-\frac{\mathrm{i} \pi \kappa_{2}^{a}}{\omega_{1} \omega_{2}} \sum_{j=1}^{N_{a}} X_{a, j}^{2} \mathrm{e}^{\frac{2 \pi \mathrm{i} \kappa_{1}^{a}}{\omega_{1} \omega_{2}} \sum_{j=1}^{N_{a}} X_{a, j}}} \\
& \times \Delta_{S}\left(\underline{X}_{a}\right) \prod_{j=1}^{N_{1}} \prod_{k=1}^{N_{2}} \frac{S_{2}\left(\omega-M_{21}+X_{2, k}-X_{1, j} \mid \underline{\omega}\right) S_{2}\left(\beta \omega+M_{12}+X_{2, k}-X_{1, j} \mid \underline{\omega}\right)}{S_{2}\left(\omega-\omega \beta-M_{21}+X_{2, k}-X_{1, j} \mid \underline{\omega}\right) S_{2}\left(M_{12}+X_{2, k}-X_{1, j} \mid \underline{\omega}\right)} \\
& \times \bigotimes_{i=1,2} \exp \left(\sum_{n>0} \frac{\sum_{j=1}^{N_{a}}\left(w_{a, j}^{n}\right)_{i}}{q_{i}^{n / 2}-q_{i}^{-n / 2}} \mathrm{a}_{-n, i}^{a}-\sum_{n>0} \frac{\kappa_{2}^{a}(-1)^{n}}{\left(q_{i}^{n / 2}-q_{i}^{-n / 2}\right)\left(t_{i}^{n / 2}-t_{i}^{-n / 2}\right)} \lambda_{-n, i}\right) \\
& \times \mathrm{e}^{\sqrt{\beta} N_{a} \mathrm{Q}_{i}^{a}}|\underline{\alpha}\rangle,
\end{aligned}
$$

up to proportionality factors, with $\kappa_{1}^{a}=\omega \sqrt{\beta}\left((-1)^{a} \sqrt{\beta}\left(N_{2}-N_{1}\right)-Q_{\beta}+\alpha_{a}\right)$. Using the representation

$$
\begin{aligned}
\mathrm{a}_{-n, i}^{a} & \simeq\left(q_{i}^{\frac{n}{2}}-q_{i}^{-\frac{n}{2}}\right) \tau_{n, i}^{a}, \quad \lambda_{n, i}^{a} \simeq \frac{1}{n}\left(t_{i}^{\frac{n}{2}}-t_{i}^{-\frac{n}{2}}\right) \frac{\partial}{\partial \tau_{n, i}^{b}}, \quad \mathrm{Q}_{\lambda, i}^{a} \simeq \tau_{0, i}^{a}, \\
\mathrm{P}_{i}^{a} & =\frac{\partial}{\partial \tau_{0, i}^{a}}, \quad n>0
\end{aligned}
$$

this state describes the generating function of the mass deformed ABJ theory coupled to two additional adjoint chiral multiplets and FI parameters

$$
\begin{aligned}
& \prod_{a=1,2}\left(\mathcal{V}_{+}^{a}\right)^{\kappa_{2}^{a}} \mathcal{Z} \prod_{a=1,2}\left(\mathcal{V}_{-}^{a}\right)^{\kappa_{2}^{a}}|\underline{\alpha}\rangle \simeq Z_{\mathrm{ABJ}}\left(\beta,\left\{M_{e}\right\},\left\{\kappa_{1}^{a}\right\} \mid\left\{\underline{\tau}_{1}^{a}, \underline{\tau}_{2}^{a}\right\}\right) \\
& \quad=\left\langle\prod_{i=1,2} \exp \left(\sum_{a, b=1}^{2} \sum_{n>0} \tau_{n, i}^{b}\left(\delta_{b a} \sum_{j=1}^{N_{a}}\left(w_{a, j}^{n}\right)_{i}-\left(C_{i}^{[n]}\right)_{b a}^{-1} p_{i}^{-n / 2} \frac{(-1)^{n} \kappa_{2}^{a}}{t_{i}^{n / 2}-t_{i}^{-n / 2}}\right)\right)\right\rangle,
\end{aligned}
$$

where

$$
\begin{aligned}
\langle 1\rangle= & \mathcal{N}_{0} \int_{i \mathbb{R}} \prod_{a=1,2} \mathrm{~d}^{N_{a}} \underline{X}_{a} \prod_{a=1,2} \mathrm{e}^{-\frac{\mathrm{i} \pi \kappa_{2}^{a}}{\omega_{1} \omega_{2}} \sum_{j=1}^{N_{a}} X_{a, j}^{2}} \mathrm{e}^{\frac{2 \pi \mathrm{i} \kappa_{1}^{a}}{\omega_{1} \omega_{2}} \sum_{j=1}^{N_{a}} X_{a, j}} \Delta_{S}\left(\underline{X}_{a}\right) \\
& \times \prod_{j=1}^{N_{1}} \prod_{k=1}^{N_{2}} \frac{S_{2}\left(\omega-M_{21}+X_{2, k}-X_{1, j} \mid \underline{\omega}\right) S_{2}\left(\beta \omega+M_{12}+X_{2, k}-X_{1, j} \mid \underline{\omega}\right)}{S_{2}\left(\omega-\omega \beta-M_{21}+X_{2, k}-X_{1, j} \mid \underline{\omega}\right) S_{2}\left(M_{12}+X_{2, k}-X_{1, j} \mid \underline{\omega}\right)}, \\
\mathcal{N}_{0}= & \mathrm{e}^{-\frac{\mathrm{i} \pi \omega \beta N_{1} N_{2}}{\omega_{1} \omega_{2}}\left(M_{12}-M_{21}\right)} \prod_{i=1,2} \mathrm{e}^{\sum_{a, b=1}^{2} \tau_{0, i}^{b}\left(\delta_{a b} \sqrt{\beta} N_{a}+\left(C_{i}^{[0]}\right)_{b a}^{-1} \alpha_{a}\right)} .
\end{aligned}
$$


We can now specialize to $\beta=1 / 2$, which effectively removes the adjoint chiral multiplets and reduces $\Delta_{S}\left(\underline{X}_{a}\right)$ to pure vector contributions (see Sect. 5.2)

$$
\left.\Delta_{S}\left(\underline{X}_{a}\right)\right|_{\beta=\frac{1}{2}}=\prod_{j, k=1}^{N_{a}} S_{2}\left(X_{a, j}-X_{a, k} \mid \underline{\omega}\right)=\prod_{i=1,2} \prod_{1 \leq j<k \leq N_{a}} 2 \mathrm{i} \sin \left(\pi \frac{X_{a, j}-X_{a, k}}{\omega_{i}}\right) .
$$

Moreover, if we set

$$
\kappa_{2}^{1}=-\kappa_{2}^{2}=\kappa_{2}, \quad \kappa_{1}^{1}=\kappa_{1}^{2}=0, \quad M_{12}=M_{21}=\frac{\omega}{4},
$$

the CS levels have opposite signs, the FI parameters are set to zero and the bifundamentals are massless, and hence we are effectively describing the ABJ theory. This $\mathrm{W}_{q, t}(\Gamma)$ modular double description would allow us to write explicitly the Ward identities satisfied by ABJ generating function by acting with the algebra generators.

\section{Summary, Comments and Outlook}

In this work we have shown that a wide class of $3 \mathrm{~d} \mathcal{N}=2$ unitary quiver gauge theories on compact spaces hides a modular double $\mathrm{W}_{q, t}$ symmetry, which we defined. Our argument was based on the realization of supersymmetric Wilson loop generating functions as Fock states obtained through the action of vertex operators and screening charges of the modular double on a vacuum state. Our interpretation implies the existence of two SL(2, $\mathbb{Z}$ )-related commuting sets of $\mathrm{W}_{q, t}$ constraints (Ward identities) annihilating the YM generating functions, corresponding to highest weight conditions. As recently stressed in [84], these type of deformed Ward identities may be regarded as a further step towards a proper definition of $q$-CFT theories, a deformation of ordinary $2 \mathrm{~d}$ CFTs. While most of the studies have so far focused on the chiral description, our work shows that it is possible to consistently couple different chiral sectors into well-defined modular invariant objects [52,53]. This is familiar in 2d CFTs, where the invariance w.r.t the Moore-Seiberg groupoid puts severe constraints on the physical theories [126]. From this perspective it is not totally surprising that the structure of the modular double is dictated by the compact space geometries: in fact, localization on spaces with boundaries (see [102,127-129] for recent discussions) is notoriously more complicated than on closed spaces where there are no ambiguities due to the boundary (indeed, most of the gauge theory dualities have been tested by using compact space observables).

There are a number of further directions worth studying, physically and mathematically. First of all, the $\mathrm{W}_{q, t}$ modular double symmetry of $3 \mathrm{~d} \mathcal{N}=2$ quiver gauge theories on compact spaces that we have considered represents a new tool for studying these theories, which should supplement the existing large $N[130,131]$ or Fermi gas techniques [132-135]. Moreover, it is very likely that the $3 \mathrm{~d}$ dualities mentioned in the introduction (see also [136] for recent new results) have a natural and simple interpretation in $\mathrm{W}_{q, t}$ language, perhaps along the lines of [137] in the more familiar 4d AGT context. It is also interesting to observe the appearance of W-like symmetries in these theories, which are usually associated to area-preserving diffeomorphisms of membranes (a possible connection between $\mathrm{W}_{q, t}$ algebras and the physics of membranes was already pointed out in [109]).

Secondly, the $5 \mathrm{~d}$ gauge theory origin of the quiver $\mathrm{W}_{q, t}$ algebras immediately raises the question whether our $3 \mathrm{~d}$ gauge theory inspired construction of the modular double 
can describe the parent $5 \mathrm{~d}$ theories on compact spaces as well. While in the chiral case (i.e., $5 \mathrm{~d}$ theories on $\mathbb{R}^{4} \times S^{1}$ ) the answer is clearly affirmative according to the results of $[54,68,73,96]$, in the non-chiral case (i.e., $5 \mathrm{~d}$ theories on compact spaces) the answer is not straightforward (the rank 1 case was studied in [52,53]). First of all, the free boson realization of $\mathrm{W}_{q, t}$ explicitly breaks the $q, t$ symmetry of the $5 \mathrm{~d}$ theory, which is however restored in the large $N$ limit (i.e., sending the number of screening currents to infinity or going to the affine case). Secondly, 5d partition functions on $S^{4} \times S^{1}$ [138-140], $S^{5}[97,141-146], Y^{p, q}[147,148]$ and toric Sasaki-Einstein manifolds [149] exhibit an $\operatorname{SL}(3, \mathbb{Z})$ factorization property rather than just $\operatorname{SL}(2, \mathbb{Z})$. How this symmetry enhancement can emerge from $3 \mathrm{~d}$ considerations is highly non-trivial and we leave this topic for future research. Here we just observe that the large $N$ limit we have just mentioned is essentially the geometric transition in open/closed topological strings $[6,121,150,151]$. Interestingly enough, the $S^{3}$ and $S^{5}$ partition functions have been proposed [97] to give a non-perturbative definition of open and closed topological string partition functions respectively, which is consistent with the expectation that our construction can describe $5 \mathrm{~d}$ theories on compact spaces as well.

Thirdly, the $\mathrm{W}_{q, t}$ modular double algebra might also be useful to study $4 \mathrm{~d}$ supersymmetric gauge theories in all those situations where $3 \mathrm{~d}$ theories appear as boundary conditions or interfaces $[12,13,15,152-154]$. In particular, S-duality domain wall in 4d $\mathcal{N}=2$ theories of class $\mathcal{S}[155]$ are realized by $3 \mathrm{~d} \mathcal{N}=2 \mathrm{U}(N)$ YM theories on $S_{b}^{3}[156-$ 160], and their partition functions are modular kernels of Liouville [161-163] or Toda theories [160]. Moreover, our construction of the $\mathrm{W}_{q, t}$ modular double should easily lift to the elliptic case $[164,165]$. In fact, as shown in [164], 4d holomorphic blocks [67] of $4 \mathrm{~d} \mathcal{N}=1 \mathrm{U}(N)$ theories on $D^{2} \times \mathbb{T}^{2}$ are captured by correlators of vertex operators and screening charges of the elliptic Virasoro algebra. Since $4 \mathrm{~d}$ compact space partition functions (including $\mathcal{N}=1,2$ supersymmetric indexes [166-169], see also [170] for a review) can be decomposed into holomorphic blocks as much as in 3d [67,171-173], we expect that elliptic matrix models provided by Coulomb branch localization on $4 \mathrm{~d}$ manifolds with the topology of $S^{3} \times S^{1}, L(r, 1) \times S^{1}$ and $S^{2} \times \mathbb{T}^{2}$ [40,174-179] can naturally be studied with the same techniques developed in this paper and [118]. This perspective might also reveal interesting connections between the elliptic W algebras and elliptic integrable systems arising in that context [180-182].

Finally, there are mathematical aspects that deserve further investigations. For instance, our construction of the $\mathrm{W}_{q, t}$ modular double shares many similarities with Faddeev's $\mathcal{U}_{q}\left(\mathfrak{s l}_{2}\right)$ modular double [183] (see also [184]). In that case there are two commuting copies of $\mathcal{U}_{q}\left(\mathfrak{s l}_{2}\right)$ whose $q$-deformation parameters are related by $S \in \operatorname{SL}(2, \mathbb{Z})$ and a unique simultaneous $\mathcal{R}$ matrix for both copies; in our case there are two commuting $\mathrm{W}_{q, t}$ algebras with $q$-deformation parameters related by $\operatorname{SL}(2, \mathbb{Z})$ elements and a unique simultaneous screening current $\mathcal{S}$ for both copies. Interestingly enough, they are exactly the representations of the $\mathcal{U}_{q}\left(\mathfrak{s l}_{2}\right)$ modular double that are relevant in the construction of the Liouville modular kernel, which has also a $3 \mathrm{~d}$ gauge theory interpretation as we mentioned. Therefore, we also hope that our results may help to clarify the precise relation between $\mathcal{U}_{q}\left(\mathfrak{s l}_{2}\right)$ and $q$-Virasoro algebras (and their modular doubles), which is still not very well understood, as well as the role of $\mathrm{W}_{q, t}$ algebras in ordinary $2 \mathrm{~d}$ CFTs.

Acknowledgements. We thank Francesco Bonechi, Sara Pasquetti, Vasily Pestun and Alessandro Torrielli for discussions. The research of A.N. is supported in part by INFN and by MIUR-FIRB Grant RBFR10QS5J "String Theory and Fundamental Interactions". The research of F.N. and M.Z. is supported in part by Vetenskapsrådet under Grant \#2014-5517, by the STINT grant and by the grant "Geometry and Physics" from the Knut and Alice Wallenberg foundation. 
Open Access This article is distributed under the terms of the Creative Commons Attribution 4.0 International License (http://creativecommons.org/licenses/by/4.0/), which permits unrestricted use, distribution, and reproduction in any medium, provided you give appropriate credit to the original author(s) and the source, provide a link to the Creative Commons license, and indicate if changes were made.

\section{A. Special Functions}

We summarize the special functions and their properties used throughout the paper, for details we refer to [185]. The (multiple) $q$-Pochhammer symbol is defined by

$$
\left(x ; q_{1}, \ldots, q_{n}\right)_{\infty}=\exp \left(-\sum_{k>0} \frac{x^{k}}{k \prod_{i=1}^{n}\left(1-q_{i}^{k}\right)}\right)=\prod_{k_{1}, \ldots, k_{n} \geq 0}\left(1-x q_{1}^{k_{1}} \cdots q_{n}^{k_{n}}\right) .
$$

The last expression is valid for $\left|q_{i}\right|<1$, but it can be continued to other regions by means of

$$
(x ; q)_{\infty}=\frac{1}{\left(q^{-1} x ; q^{-1}\right)_{\infty}} .
$$

The finite $q$-Pochhammer symbol is defined by

$$
(x ; q)_{n}=\frac{(x ; q)_{\infty}}{\left(q^{n} x ; q\right)_{\infty}}=\prod_{k=1}^{n-1}\left(1-x q^{k}\right),
$$

and satisfies

The $\Theta$ function is defined by

$$
(x ; q)_{-n}=\left(q^{-n} x ; q\right)_{n}^{-1} .
$$

$$
\Theta(x ; q)=(x ; q)_{\infty}\left(q x^{-1} ; q\right)_{\infty} .
$$

The modular properties we are interested in are

$$
\begin{aligned}
& \Theta\left(\mathrm{e}^{2 \pi \mathrm{i} X} \mathrm{e}^{\frac{2 \pi \mathrm{i} \ell}{r}} ; \mathrm{e}^{2 \pi \mathrm{i} \epsilon}\right) \Theta\left(\mathrm{e}^{\frac{2 \pi \mathrm{i} X}{r \epsilon-1}} \mathrm{e}^{-\frac{2 \pi \mathrm{i} \ell}{r}} ; \mathrm{e}^{\frac{2 \pi \mathrm{i} \epsilon}{r \epsilon-1}}\right) \\
& =\mathrm{e}^{\frac{\mathrm{i} \pi}{r} \ell(r-\ell)} \mathrm{e}^{-\mathrm{i} \pi\left(B_{22}(X \mid 1, \epsilon)+B_{22}\left(1+\frac{X}{r \epsilon-1} \mid 1, \frac{\epsilon}{r \epsilon-1}\right)\right)},
\end{aligned}
$$

for $r \in \mathbb{Z}, \ell \in \mathbb{Z}_{r}$, and

$$
\begin{gathered}
\Theta\left(q^{-\frac{\ell}{2}} x ; q\right) \Theta\left(q^{-\frac{\ell}{2}} x^{-1} ; q^{-1}\right)=\left(-q^{-\frac{1}{2}} x\right)^{\ell}, \\
\Theta\left(q^{-\frac{\ell}{2}} x ; q\right) \Theta\left(q^{\frac{\ell}{2}} x ; q^{-1}\right)=(-x)^{\ell+1},
\end{gathered}
$$

for $\ell \in \mathbb{Z}$. Here $B_{22}(X \mid \underline{\omega})$ is the quadratic Bernoulli polynomial

$$
B_{22}(X \mid \underline{\omega})=\frac{1}{\omega_{1} \omega_{2}}\left(\left(X-\frac{\omega}{2}\right)^{2}-\frac{\omega_{1}^{2}+\omega_{2}^{2}}{12}\right), \quad \omega=\omega_{1}+\omega_{2} .
$$

The double Sine function $S_{2}(X \mid \underline{\omega})$ is defined as the $\zeta$-regularized product

$$
S_{2}(X \mid \underline{\omega})=\prod_{n_{1}, n_{2} \geq 0} \frac{n_{1} \omega_{1}+n_{2} \omega_{2}+X}{n_{1} \omega_{1}+n_{2} \omega_{2}+\omega-X} .
$$

Two important properties of the double Sine function are the quasi-periodicity

$$
\begin{aligned}
\frac{S_{2}(X \mid \underline{\omega})}{S_{2}\left(b_{1} \omega_{1}+b_{2} \omega_{2}+X \mid \underline{\omega}\right)}= & (-1)^{b_{1} b_{2}} 2^{b_{1}+b_{2}} \prod_{n_{1}=0}^{b_{1}-1} \sin \left(\pi \frac{n_{1} \omega_{1}+X}{\omega_{2}}\right) \\
& \times \prod_{n_{2}=0}^{b_{2}-1} \sin \left(\pi \frac{n_{2} \omega_{2}+X}{\omega_{1}}\right)
\end{aligned}
$$


and the reflection

$$
S_{2}(X \mid \underline{\omega}) S_{2}(\omega-X \mid \underline{\omega})=1 .
$$

For two generic complex numbers $\underline{\omega}$ such that $\operatorname{Im}\left(\frac{\omega_{2}}{\omega_{1}}\right) \neq 0$, the double Sine function has the factorized expression

$$
S_{2}(X \mid \underline{\omega})=\mathrm{e}^{\frac{\mathrm{i} \pi}{2} B_{22}(X \mid \underline{\omega})}\left(\mathrm{e}^{\frac{2 \pi \mathrm{i}}{\omega_{1}} X} ; \mathrm{e}^{2 \pi \mathrm{i} \frac{\omega}{\omega_{1}}}\right)_{\infty}\left(\mathrm{e}^{\frac{2 \pi \mathrm{i}}{\omega_{2}} X} ; \mathrm{e}^{2 \pi \mathrm{i} \frac{\omega}{\omega_{2}}}\right)_{\infty} .
$$

The generalized double Sine function $S_{2, \ell}(X \mid \underline{\omega})$ is [67]

$$
S_{2, \ell}(X \mid \underline{\omega})=S_{2}\left(X+\omega_{1}\left(r-[\ell]_{r}\right) \mid \omega, r \omega_{1}\right) S_{2}\left(X+\omega_{2}[\ell]_{r} \mid \omega, r \omega_{2}\right),
$$

with $[\ell]_{r}$ the positive integer part of $\ell \bmod r$. For two generic complex numbers $\underline{\omega}$ such that $\operatorname{Im}\left(\frac{\omega_{2}}{\omega_{1}}\right) \neq 0$, we also have the factorized expression

$$
\begin{aligned}
S_{2, \ell}(X \mid \underline{\omega})=\mathrm{e}^{-\frac{\mathrm{i} \pi}{2 r}[\ell]_{r}\left(r-[\ell]_{r}\right)} \mathrm{e}^{\frac{\mathrm{i} \pi}{2}\left(B_{22}\left(X \mid \omega, r \omega_{1}\right)+B_{22}\left(X+r \omega_{2} \mid \omega, r \omega_{2}\right)\right)} & \\
& \times\left(\mathrm{e}^{\frac{2 \pi \mathrm{i}}{r \omega_{1}}\left(X+\omega_{1} \ell\right)} ; \mathrm{e}^{2 \pi \mathrm{i} \frac{\omega}{r \omega_{1}}}\right)_{\infty}\left(\mathrm{e}^{\frac{2 \pi \mathrm{i}}{r \omega_{2}}\left(X-\omega_{2} \ell\right)} ; \mathrm{e}^{2 \pi \mathrm{i} \frac{\omega}{r \omega_{2}}}\right)_{\infty} .
\end{aligned}
$$

\section{References}

1. Witten, E.: Quantum field theory and the jones polynomial. Commun. Math. Phys. 121, 351-399 (1989)

2. Aganagic, M., Shakirov, S.: Knot homology and refined Chern-Simons index. Commun. Math. Phys. 333, 187-228. arXiv:1105.5117 (2015)

3. Aganagic, M., Shakirov, S.: Refined Chern-Simons theory and knot homology. Proc. Symp. Pure Math. 85, 3-32. arXiv:1202.2489 (2012)

4. Aganagic, M., Shakirov, S.: Refined Chern-Simons theory and topological string. arXiv:1210.2733 (2012)

5. Witten, E.: Chern-Simons gauge theory as a string theory. Prog. Math. 133, 637678. arXiv:hep-th/9207094 (1995)

6. Gopakumar, R., Vafa, C.: On the gauge theory/geometry correspondence. Adv. Theor. Math. Phys. 3, 1415-1443. arXiv:hep-th/9811131 (1999)

7. Aganagic, M., Marino, M., Vafa, C.: All loop topological string amplitudes from Chern-Simons theory. Commun. Math. Phys. 247, 467-512. arXiv:hep-th/0206164 (2004)

8. Hanany, A., Witten, E.: Type IIB superstrings, BPS monopoles, and three-dimensional gauge dynamics. Nucl. Phys. B 492, 152-190. arXiv:hep-th/9611230 (1997)

9. Aharony, O., Bergman, O., Jafferis, D.L., Maldacena, J.: N = 6 superconformal chern-simons-matter theories, m2-branes and their gravity duals. JHEP 10, 091. arXiv:0806.1218 (2008)

10. Aharony, O., Bergman, O., Jafferis, D.L.: Fractional M2-branes. JHEP 11, 043. arXiv:0807.4924 (2008)

11. Dimofte, T., Gukov, S., Hollands, L.: Vortex counting and Lagrangian 3-manifolds. Lett. Math. Phys. 98, 225-287. arXiv:1006.0977 (2011)

12. Dimofte, T., Gaiotto, D., Gukov, S.: Gauge theories labelled by three-manifolds. Commun. Math. Phys. 325, 367-419. arXiv:1108.4389 (2014)

13. Dimofte, T., Gaiotto, D., Gukov, S.: 3-Manifolds and 3d indices. Adv. Theor. Math. Phys. 17, 9751076 arXiv:1112.5179 (2013)

14. Chung, H.-J., Dimofte, T., Gukov, S., Sulkowski, P.: 3d-3d correspondence revisited. JHEP. 04, 140. arXiv:1405.3663 (2016)

15. Dimofte, T.: 3d Superconformal theories from three-manifolds. In: Teschner, J. (ed). New Dualities of Supersymmetric Gauge Theories, pp. 339-373. arXiv:1412.7129 (2016)

16. Witten, E.: Quantization of Chern-Simons gauge theory with complex gauge group. Commun. Math. Phys. 137, 29-66 (1991)

17. Karch, A.: Seiberg duality in three-dimensions. Phys. Lett. B 405, 79-84. arXiv:hep-th/9703172 (1997)

18. Aharony, O.: IR duality in $\mathrm{d}=3 \mathrm{~N}=2$ supersymmetric $\mathrm{USp}(2 \mathrm{~N}(\mathrm{c}))$ and $\mathrm{U}(\mathrm{N}(\mathrm{c}))$ gauge theories. Phys. Lett. B 404, 71-76. arXiv:hep-th/9703215 (1997)

19. Niarchos, V.: Seiberg duality in Chern-Simons theories with fundamental and adjoint matter. JHEP 11, 001. arXiv:0808.2771 (2008)

20. Benini, F., Closset, C., Cremonesi, S.: Comments on 3d Seiberg-like dualities. JHEP. 10, 075. arXiv:1108.5373 (2011)

21. Aharony, O., Razamat, S.S., Seiberg, N., Willett, B.: 3d dualities from 4d dualities. JHEP. 07, 149. arXiv:1305.3924 (2013) 
22. Aharony, O., Razamat, S.S., Seiberg, N., Willett, B.: $3 d$ dualities from $4 d$ dualities for orthogonal groups. JHEP 08, 099. arXiv:1307.0511 (2013)

23. Intriligator, K.A., Seiberg, N.: Mirror symmetry in three-dimensional gauge theories. Phys. Lett. B 387, 513-519. arXiv:hep-th/9607207 (1996)

24. de Boer, J., Hori, K., Oz, Y., Yin, Z.: Branes and mirror symmetry in $\mathrm{n}=2$ supersymmetric gauge theories in three-dimensions. Nucl. Phys. B 502, 107-124. arXiv:hep-th/9702154 (1997)

25. Aharony, O., Hanany, A., Intriligator, K.A., Seiberg, N., Strassler, M.J.: Aspects of $\mathrm{n}=2$ supersymmetric gauge theories in three-dimensions. Nucl. Phys. B 499, 67-99. arXiv:hep-th/9703110 (1997)

26. Gadde, A., Gukov, S., Putrov, P.: Fivebranes and 4-manifolds. In: Ballmann, W., Blohmann, C., Faltings, G., Teichner, P., Zagier, D. (eds). Arbeitstagung Bonn 2013, pp. 155-245. arXiv:1306.4320 (2016)

27. Gukov, S., Pei, D.: Equivariant Verlinde formula from fivebranes and vortices. arXiv:1501.01310 (2015)

28. Festuccia, G., Seiberg, N.: Rigid supersymmetric theories in curved superspace. JHEP 06, 114. arXiv:1105.0689 (2011)

29. Dumitrescu, T.T., Festuccia, G., Seiberg, N.: Exploring curved superspace. JHEP 08, 141. arXiv:1205.1115 (2012)

30. Closset, C., Dumitrescu, T.T., Festuccia, G., Komargodski, Z.: Supersymmetric field theories on three-manifolds. JHEP 05, 017. arXiv:1212.3388 (2013)

31. Closset, C., Dumitrescu, T.T., Festuccia, G., Komargodski, Z.: The geometry of supersymmetric partition functions. JHEP 01, 124. arXiv:1309.5876 (2014)

32. Closset, C., Dumitrescu, T.T., Festuccia, G., Komargodski, Z.: From rigid supersymmetry to twisted holomorphic theories. Phys. Rev. D 90, 085006. arXiv:1407.2598 (2014)

33. Imbimbo, C., Rosa, D.: Topological anomalies for Seifert 3-manifolds. JHEP $07,068$. arXiv:1411.6635 (2015)

34. Pestun, V.: Localization of gauge theory on a four-sphere and supersymmetric Wilson loops. Commun. Math. Phys. 313, 71-129. arXiv:0712.2824 (2012)

35. Kapustin, A., Willett, B., Yaakov, I.: Exact results for wilson loops in superconformal chern-simons theories with matter. JHEP 03, 089. arXiv:0909.4559 (2010)

36. Hama, N., Hosomichi, K., Lee, S.: SUSY gauge theories on squashed three-spheres. JHEP $05,014$. arXiv:1102.4716 (2011)

37. Imamura, Y., Yokoyama, D.: $\mathrm{N}=2$ supersymmetric theories on squashed three-sphere. Phys. Rev. D 85, 025015. arXiv:1109.4734 (2012)

38. Alday, L.F., Martelli, D., Richmond, P., Sparks, J.: Localization on three-manifolds. JHEP 10, 095 arXiv:1307.6848 (2013)

39. Gang, D.: Chern-Simons theory on L(p,q) lens spaces and localization. arXiv:0912.4664 (2009)

40. Benini, F., Nishioka, T., Yamazaki, M.: 4d Index to 3d Index and 2d TQFT. Phys. Rev. D 86, 065015. arXiv:1109.0283 (2012)

41. Alday, L.F., Fluder, M., Sparks, J.: The large N limit of M2-branes on lens spaces. JHEP 10, 057. arXiv:1204.1280 (2012)

42. Imamura, Y., Yokoyama, D.: $S^{3} / \mathbb{Z}_{n}$ partition function and dualities. JHEP $11,122$. arXiv:1208.1404 (2012)

43. Imamura, Y., Matsuno, H., Yokoyama, D.: Factorization of the $S^{3} / \mathbb{Z}_{n}$ partition function. Phys. Rev. D 89, 085003. arXiv:1311.2371 (2014)

44. Imamura, Y., Yokoyama, S.: Index for three dimensional superconformal field theories with general r-charge assignments. JHEP 04, 007. arXiv:1101.0557 (2011)

45. Kapustin, A., Willett, B.: Generalized Superconformal Index for Three Dimensional Field Theories. arXiv:1106.2484 (2011)

46. Benini, F., Zaffaroni, A.: A topologically twisted index for three-dimensional supersymmetric theories. JHEP 07, 127. arXiv:1504.03698 (2015)

47. Benini, F., Zaffaroni, A.: Supersymmetric partition functions on Riemann surfaces. arXiv:1605.06120 (2016)

48. Shiraishi, J., Kubo, H., Awata, H., Odake, S.: A quantum deformation of the virasoro algebra and the macdonald symmetric functions. Lett. Math. Phys. 38, 33-51. arXiv:q-alg/9507034 (1996)

49. Alday, L.F., Gaiotto, D., Tachikawa, Y.: Liouville correlation functions from four-dimensional gauge theories. Lett. Math. Phys. 91, 167-197. arXiv:0906.3219 (2010)

50. Wyllard, N.: A(n-1) conformal toda field theory correlation functions from conformal $\mathrm{n}=2$ su(n) quiver gauge theories. JHEP 11, 002. arXiv:0907.2189 (2009)

51. Awata, H., Yamada, Y.: Five-dimensional AGT conjecture and the deformed Virasoro algebra. JHEP 01, 125. arXiv:0910.4431 (2010)

52. Nieri, F., Pasquetti, S., Passerini, F.: $3 d$ and $5 d$ gauge theory partition functions as $q$-deformed CFT correlators. Lett. Math. Phys. 105, 109-148. arXiv:1303.2626 (2015)

53. Nieri, F., Pasquetti, S., Passerini, F., Torrielli, A.: 5d partition functions, q-virasoro systems and integrable spin-chains. JHEP 12, 040. arXiv:1312.1294 (2014) 
54. Aganagic, M., Haouzi, N.: ADE Little String Theory on a Riemann Surface (and Triality). arXiv:1506.04183 (2015)

55. Aganagic, M., Cheng, M.C.N., Dijkgraaf, R., Krefl, D., Vafa, C.: Quantum geometry of refined topological strings. JHEP. 11, 019. arXiv:1105.0630 (2012)

56. Gadde, A., Gukov, S., Putrov, P.: Walls, lines, and spectral dualities in 3d gauge theories. JHEP 05, 047. arXiv:1302.0015 (2014)

57. Gaiotto, D., Koroteev, P.: On three dimensional quiver gauge theories and integrability. JHEP 05, 126. arXiv:1304.0779 (2013)

58. Bullimore, M., Kim, H.-C., Koroteev, P.: Defects and quantum Seiberg-Witten geometry. JHEP 05, 095. arXiv:1412.6081 (2015)

59. Koroteev, P., Sciarappa, A.: Quantum Hydrodynamics from Large-n Supersymmetric Gauge Theories. arXiv: 1510.00972 (2016)

60. Koroteev, P., Sciarappa, A.: On elliptic algebras and large-n supersymmetric gauge theories. J. Math. Phys. 57, 112302. arXiv:1601.08238 (2016)

61. Pasquetti, S.: Factorisation of $\mathrm{N}=2$ theories on the squashed 3 -sphere. JHEP 04, 120. arXiv:1111.6905 (2012)

62. Beem, C., Dimofte, T., Pasquetti, S.: Holomorphic blocks in three dimensions. JHEP 12, 177. arXiv:1211.1986 (2014)

63. Benini, F., Peelaers, W.: Higgs branch localization in three dimensions. JHEP $05,030$. arXiv:1312.6078 (2014)

64. Fujitsuka, M., Honda, M., Yoshida, Y.: Higgs branch localization of $3 \mathrm{~d} N=2$ theories. PTEP 2014, 123B02. arXiv:1312.3627 (2014)

65. Taki, M.: Holomorphic Blocks for 3d Non-abelian Partition Functions. arXiv:1303.5915 (2013)

66. Hwang, C., Park, J.: Factorization of the 3d superconformal index with an adjoint matter. JHEP 11, 028. arXiv:1506.03951 (2015)

67. Nieri, F., Pasquetti, S.: Factorisation and holomorphic blocks in 4d. JHEP 11, 155. arXiv:1507.00261 (2015)

68. Aganagic, M., Haouzi, N., Kozcaz, C., Shakirov, S.: Gauge/Liouville Triality. arXiv:1309.1687 (2013)

69. Aganagic, M., Shakirov, S.: Gauge/Vortex duality and AGT. In: Teschner, J. (ed.) New Dualities of Supersymmetric Gauge Theories, pp. 419-448. arXiv:1412.7132 (2016)

70. Nekrasov, N.: Bps/cft correspondence: non-perturbative Dyson-Schwinger equations and qqcharacters. JHEP 03, 181. arXiv:1512.05388 (2016)

71. Nekrasov, N., Pestun, V.: Seiberg-Witten Geometry of Four Dimensional N=2 Quiver Gauge Theories. arXiv:1211.2240 (2012)

72. Nekrasov, N., Pestun, V., Shatashvili, S.: Quantum Geometry and Quiver Gauge Theories. arXiv:1312.6689 (2013)

73. Kimura, T., Pestun, V.: Quiver W-algebras. arXiv:1512.08533 (2015)

74. Nekrasov, N.A.: Seiberg-Witten prepotential from instanton counting. Adv. Theor. Math. Phys. 7, 831864. arXiv:hep-th/0206161 (2003)

75. Nekrasov, N., Okounkov, A.: Seiberg-Witten theory and random partitions. Prog. Math. 244, 525596. arXiv:hep-th/0306238 (2006)

76. Leung, N.C., Vafa, C.: Branes and toric geometry. Adv. Theor. Math. Phys. 2, 91-118. arXiv:hep-th/9711013 (1998)

77. Kol, B., Rahmfeld, J.: BPS spectrum of five-dimensional field theories, (p,q) webs and curve counting. JHEP 08, 006. arXiv:hep-th/9801067 (1998)

78. Aharony, O., Hanany, A.: Branes, superpotentials and superconformal fixed points. Nucl. Phys. B 504, 239-271. arXiv:hep-th/9704170 (1997)

79. Aharony, O., Hanany, A., Kol, B.: Webs of $(p, q)$ five-branes, five-dimensional field theories and grid diagrams. JHEP 01, 002. arXiv:hep-th/9710116 (1998)

80. Iqbal, A., Kozcaz, C., Vafa, C.: The Refined topological vertex. JHEP 10, 069. arXiv:hep-th/0701156 (2009)

81. Awata, H., Kanno, H.: Instanton counting, Macdonald functions and the moduli space of Dbranes. JHEP 05, 039. arXiv:hep-th/0502061 (2005)

82. Taki, M.: Refined topological vertex and instanton counting. JHEP 03, 048. arXiv:0710.1776 (2008)

83. Mironov, A., Morozov, A., Zenkevich, Y.: Ding-Iohara-Miki symmetry of network matrix models. Phys. Lett. B 762, 196-208. arXiv:1603.05467 (2016)

84. Awata, H., Kanno, H., Matsumoto, T., Mironov, A., Morozov, A., Morozov, A. et al.: Explicit examples of DIM constraints for network matrix models. JHEP 07, 103. arXiv:1604.08366 (2016)

85. Ding, J., Iohara, K.: Generalization and Deformation of Drinfeld Quantum Affine Algebras. arXiv:q-alg/9608002 (1996)

86. Miki, K.: A (q, $\gamma)$ analog of the $\mathrm{W}_{1+\text { ? }}$ algebra. J. Math. Phys. 48, 123520-123520 (2007)

87. Iqbal, A., Kashani-Poor, A.-K.: The vertex on a strip. Adv. Theor. Math. Phys. 10, 317-343. arXiv:hep-th/0410174 (2006) 
88. Awata, H., Yamada, Y.: Five-dimensional AGT Relation and the deformed beta-ensemble. Prog. Theor. Phys. 124, 227-262. arXiv:1004.5122 (2010)

89. Mironov, A., Morozov, A., Shakirov, S., Smirnov, A.: Proving AGT conjecture as HS duality: extension to five dimensions. Nucl. Phys. B 855, 128-151. arXiv:1105.0948 (2012)

90. Carlsson, E., Nekrasov, N., Okounkov, A.: Five Dimensional Gauge Theories and Vertex Operators. arXiv:1308.2465 (2013)

91. Zenkevich, Y.: Generalized macdonald polynomials, spectral duality for conformal blocks and agt correspondence in five dimensions. JHEP 05, 131. arXiv:1412.8592 (2015)

92. Zenkevich, Y.: Quantum Spectral Curve for (q,t)-matrix Model. arXiv:1507.00519 (2015)

93. Morozov, A., Zenkevich, Y.: Decomposing Nekrasov decomposition. JHEP 02, 098. arXiv:1510.01896 (2016)

94. Mironov, A., Morozov, A., Zenkevich, Y.: On elementary proof of AGT relations from six dimensions. Phys. Lett. B 756, 208-211. arXiv:1512.06701 (2016)

95. Mironov, A., Morozov, A., Zenkevich, Y.: Spectral duality in elliptic systems, six-dimensional gauge theories and topological strings. JHEP 05, 121. arXiv:1603.00304 (2016)

96. Aganagic, M., Haouzi, N., Shakirov, S.: $A_{n}$-Triality. arXiv:1403.3657 (2014)

97. Lockhart, G., Vafa, C.: Superconformal Partition Functions and Non-perturbative Topological Strings. arXiv:1210.5909 (2012)

98. Morozov, A.: Integrability and matrix models. Phys. Usp. 37, 1-55. arXiv:hep-th/9303139 (1994)

99. Di Francesco, P., Ginsparg, P.H., Zinn-Justin, J.: 2-D gravity and random matrices. Phys. Rept. 254, 1133. arXiv:hep-th/9306153 (1995)

100. Odake, S.: Beyond CFT: Deformed Virasoro and elliptic algebras. In: Theoretical Physics at the End of the Twentieth Century. Proceedings, Summer School, Banff, Canada, June 27-July 10, 1999, pp. 307-449 (1999). arXiv:hep-th/9910226

101. Itoyama, H., Oota, T., Yoshioka, R.: q -vertex operator from 5d nekrasov function. J. Phys. A Math. Theor. 49, 345201 (2016)

102. Yoshida, Y., Sugiyama, K.: Localization of $3 \mathrm{~d} \mathcal{N}=2$ supersymmetric theories on $s^{1} \times d^{2}$. arXiv:1409.6713 (2014)

103. Benini, F., Eager, R., Hori, K., Tachikawa, Y.: Elliptic genera of two-dimensional N = 2 gauge theories with rank-one gauge groups. Lett. Math. Phys. 104, 465-493. arXiv:1305.0533 (2014)

104. Benini, F., Eager, R., Hori, K., Tachikawa, Y.: Elliptic genera of $2 \mathrm{~d} \mathcal{N}=2$ gauge theories. Commun. Math. Phys. 333, 1241-1286. arXiv:1308.4896 (2015)

105. Gadde, A., Gukov, S.: 2d index and surface operators. JHEP 03, 080. arXiv:1305.0266 (2014)

106. Hosomichi, K.: A review on SUSY gauge theories on $\mathbf{S}^{3}$. In: J. Teschner, (ed.) New Dualities of Supersymmetric Gauge Theories, pp. 307-338. arXiv:1412.7128 (2016)

107. Jafferis, D.L.: The exact superconformal R-symmetry extremizes Z. JHEP $\mathbf{0 5 ,} 159$. arXiv:1012.3210 (2012)

108. Tanaka, A.: Comments on knotted 1/2 BPS Wilson loops. JHEP 07, 097. arXiv:1204.5975 (2012)

109. Awata, H., Kubo, H., Odake, S., Shiraishi, J.: Virasoro type symmetries in solvable models, In: Extended and Quantum Algebras and their Applications to Physics Tianjin, China, August 19-24, 1996. arXiv:hep-th/9612233 (1996)

110. Ohkubo, Y., Awata, H., Fujino, H.: Crystallization of Deformed Virasoro Algebra, Ding-Iohara-Miki Algebra and 5d agt Correspondence. arXiv:1512.08016 (2015)

111. Bouwknegt, P., Pilch, K.: The deformed virasoro algebra at roots of unity. Commun. Math. Phys. 196:249-288 (1998)

112. Itoyama, H., Oota, T., Yoshioka, R.: $2 \mathrm{~d}-4 \mathrm{~d}$ connection between $q$-virasoro/w block at root of unity limit and instanton partition function on ale space. Nucl. Phys. B 877, 506-537. arXiv:1308.2068 (2013)

113. Itoyama, H., Oota, T., Yoshioka, R.: $q$-Virasoro/W algebra at root of unity and parafermions. Nucl. Phys. B 889, 25-35. arXiv:1408.4216 (2014)

114. Frenkel, E., Reshetikhin, N.: Deformations of W-algebras Associated to Simple Lie Algebras. arXiv:q-alg/9708006 (1997)

115. Frenkel, E., Reshetikhin, N., Semenov-Tian-Shansky, M.: Drinfeld-Sokolov reduction for difference operators and deformations of -algebras i. the case of virasoro algebra. Commun. Math. Phys. 192, 605629 (1998)

116. Semenov-Tian-Shansky, A.M., Sevostyanov, V.A.: Drinfeld-sokolov reduction for differenceoperators and deformations of w-algebras ii. the general semisimple case. Commun. Math. Phys. 192, 631647 (1998)

117. Mironov, A., Morozov, A.: On the origin of Virasoro constraints in matrix models: Lagrangian approach. Phys. Lett. B 252, 47-52 (1990)

118. Nedelin, A., Zabzine, M.: q-Virasoro Constraints in Matrix Models. arXiv:1511.03471 (2015)

119. Dijkgraaf, R., Verlinde, H.L., Verlinde, E.P.: Loop equations and Virasoro constraints in nonperturbative 2-D quantum gravity. Nucl. Phys. B 348, 435-456. (1991) 
120. Marino, M.: Chern-Simons theory, matrix integrals, and perturbative three manifold invariants. Commun. Math. Phys. 253, 25-49. arXiv:hep-th/0207096 (2004)

121. Aganagic, M., Klemm, A., Marino, M., Vafa, C.: Matrix model as a mirror of Chern-Simons theory. JHEP 02, 010. arXiv:hep-th/0211098 (2004)

122. Dubinkin, O.: On the Virasoro constraints for torus knots. J. Phys. A 47, 485203. arXiv:1307.7909 (2014)

123. Iqbal, A., Kozcaz, C.: Refined Hopf link revisited. JHEP 04, 046. arXiv:1111.0525 (2012)

124. Awata, H., Kubo, H., Odake, S., Shiraishi, J.: Quantum W(N) algebras and Macdonald polynomials. Commun. Math. Phys. 179, 401-416. arXiv:q-alg/9508011 (1996)

125. Hatsuda, Y.: ABJM on ellipsoid and topological strings. JHEP 07, 026. arXiv:1601.02728 (2016)

126. Moore, G.W., Seiberg, N.: Classical and Quantum Conformal Field Theory. Commun. Math. Phys. 123, 177 (1989)

127. Sugishita, S., Terashima, S.: Exact results in supersymmetric field theories on manifolds with boundaries. JHEP 11, 021 [arXiv:1308.1973] (2013)

128. Faizal, M., Luo, Y., Smith, D.J., Tan, M.-C., Zhao, Q.: Gauge and supersymmetry invariance of $n=2$ boundary Chern-Simons theory. Nucl. Phys. B 914, 577-598. arXiv:1601.05429 (2017)

129. Aprile, F., Niarchos, V.: $\mathcal{N}=2$ supersymmetric field theories on 3-manifolds with $A$-type boundaries. JHEP 07, 176. arXiv:1604.01561 (2016)

130. Drukker, N., Marino, M., Putrov, P.: From weak to strong coupling in ABJM theory. Commun. Math. Phys. 306, 511-563. arXiv:1007.3837 (2011)

131. Drukker, N., Marino, M., Putrov, P.: Nonperturbative aspects of ABJM theory. JHEP 11, 141. arXiv:1103.4844 (2011)

132. Marino, M., Putrov, P.: ABJM theory as a Fermi gas. J. Stat. Mech. 1203, P03001. arXiv:1110.4066 (2012)

133. Codesido, S., Grassi, A., Marino, M.: Exact results in $\mathcal{N}=8$ Chern-Simons-matter theories and quantum geometry. JHEP 07, 011. arXiv:1409.1799 (2015)

134. Grassi, A., Hatsuda, Y., Marino, M.: Quantization conditions and functional equations in $\mathrm{ABJ}(\mathrm{M})$ theories. J. Phys. A 49, 115401. arXiv:1410.7658 (2016)

135. Assel, B., Drukker, N., Felix, J.: Partition functions of $3 \mathrm{~d} \hat{D}$-quivers and their mirror duals from $1 \mathrm{~d}$ free fermions. JHEP 08, 071. arXiv:1504.07636 (2015)

136. Benvenuti, S., Pasquetti, S.: $3 \mathrm{~d} N=2$ mirror symmetry, pq-webs and monopole superpotentials. JHEP 08, 136. arXiv:1605.02675 (2016)

137. Gomis, J., Le Floch, B.: M2-brane surface operators and gauge theory dualities in Toda. JHEP 04, 183. arXiv:1407.1852 (2016)

138. Kim, H.-C., Kim, S.-S., Lee, K.: 5-dim superconformal index with enhanced En global symmetry. JHEP 10, 142. arXiv:1206.6781 (2012)

139. Terashima, S.: Supersymmetric gauge theories on $S^{4}$ x $S^{1}$. Phys. Rev. D 89, 125001. arXiv:1207.2163 (2014)

140. Iqbal, A., Vafa, C.: BPS degeneracies and superconformal index in diverse dimensions. Phys. Rev. D 90, 105031. arXiv:1210.3605 (2014)

141. Källén, J., Zabzine, M.: Twisted supersymmetric 5D Yang-Mills theory and contact geometry. JHEP 05, 125. arXiv:1202.1956 (2012)

142. Källén, J., Qiu, J., Zabzine, M.: The perturbative partition function of supersymmetric 5d yang-mills theory with matter on the five-sphere. JHEP 08, 157. arXiv:1206.6008 (2012)

143. Imamura, Y: Perturbative partition function for squashed $S^{5}$. Prog. Theor. Exp. Phys. 2013(7), 073B01. arXiv:1210.6308 (2003)

144. Kim, H.-C., Kim, J., Kim, S.: Instantons on the 5-Sphere and M5-Branes. arXiv:1211.0144 (2012)

145. Kim, H.-C., Kim, S.: M5-branes from gauge theories on the 5-sphere. JHEP $\mathbf{0 5}, 144$. arXiv:1206.6339 (2013)

146. Minahan, J.A., Nedelin, A., Zabzine, M.: 5D super Yang-Mills theory and the correspondence to $\mathrm{AdS}_{7} / \mathrm{CFT}_{6}$. J. Phys. A 46, 355401. arXiv:1304.1016 (2013)

147. Qiu, J., Zabzine, M.: 5D super Yang-Mills on $Y^{p, q}$ Sasaki-Einstein manifolds. Commun. Math. Phys. 333, 861-904. arXiv:1307.3149 (2015)

148. Qiu, J., Zabzine, M.: Factorization of 5D super Yang-Mills theory on $Y^{p, q}$ spaces. Phys. Rev. D 89, 065040. arXiv:1312.3475 (2014)

149. Qiu, J., Tizzano, L., Winding, J., Zabzine, M.: Gluing Nekrasov partition functions. Commun. Math. Phys. 337, 785-816. arXiv:1403.2945 (2015)

150. Vafa, C.: Superstrings and topological strings at large N. J. Math. Phys. 42, 27982817. arXiv:hep-th/0008142 (2001)

151. Cachazo, F., Intriligator, K.A., Vafa, C.: A large $\mathrm{N}$ duality via a geometric transition. Nucl. Phys. B 603, 3-41. arXiv:hep-th/0103067 (2001)

152. Gaiotto, D., Witten, E.: Supersymmetric boundary conditions in N = 4 super Yang-Mills theory. J. Stat. Phys. 135, 789-855. arXiv:0804.2902 (2009) 
153. Gaiotto, D., Witten, E.: S-duality of boundary conditions in $\mathrm{N}=4$ super Yang-Mills theory. Adv. Theor. Math. Phys. 13, 721-896. arXiv:0807.3720 (2009)

154. Cecotti, S., Cordova, C., Vafa, C.: Braids, Walls, and Mirrors. arXiv:1110.2115 (2011)

155. Gaiotto, D.: N=2 dualities. JHEP 08, 034. arXiv:0904.2715 (2012)

156. Terashima, Y., Yamazaki, M.: SL(2,R) Chern-Simons, Liouville, and gauge theory on duality walls. JHEP 08, 135. arXiv:1103.5748 (2011)

157. Galakhov, D.V., Mironov, A., Morozov, A., Smirnov, A., Mironov, A.D., Morozov, A.Yu. et al.: Threedimensional extensions of the Alday-Gaiotto-Tachikawa relation. Theor. Math. Phys. 172, 939962. arXiv:1104.2589 (2012)

158. Teschner, J., Vartanov, G.: 6j symbols for the modular double, quantum hyperbolic geometry, and supersymmetric gauge theories. Lett. Math. Phys. 104, 527-551. arXiv:1202.4698 (2014)

159. Teschner, J., Vartanov, G.S.: Supersymmetric gauge theories, quantization of $\mathcal{M}_{\text {flat }}$, and conformal field theory. Adv. Theor. Math. Phys. 19, 1-135. arXiv:1302.3778 (2015)

160. Le Floch, B.: S-duality Wall of SQCD from Toda Braiding. arXiv:1512.09128 (2015)

161. Ponsot, B., Teschner, J.: Liouville Bootstrap Via Harmonic Analysis on a Noncompact Quantum Group. arXiv:hep-th/9911110 (1999)

162. Ponsot, B., Teschner, J.: Clebsch-Gordan and Racah-Wigner coefficients for a continuous series of representations of u(q)(sl(2,r)). Commun. Math. Phys. 224, 613-655. arXiv:math/0007097 (2001)

163. Teschner, J.: Liouville theory revisited. Class. Quant. Grav. 18, R153-R222. arXiv:hep-th/0104158 (2001)

164. Nieri, F.: An Elliptic Virasoro Symmetry in 6d. arXiv:1511.00574 (2015)

165. Iqbal, A., Kozcaz, C., Yau, S.-T.: Elliptic Virasoro Conformal Blocks. arXiv:1511.00458

166. Romelsberger, C.: Counting chiral primaries in $\mathrm{N}=1, \mathrm{~d}=4$ superconformal field theories. Nucl. Phys. B 747, 329-353. arXiv:hep-th/0510060 (2006)

167. Romelsberger, C.: Calculating the Superconformal Index and Seiberg Duality. arXiv:0707.3702 (2007)

168. Kinney, J., Maldacena, J.M., Minwalla, S., Raju, S.: An index for 4 dimensional super conformal theories. Commun. Math. Phys. 275, 209-254. arXiv:hep-th/0510251 (2007)

169. Dolan, F.A., Osborn, H.: Applications of the superconformal index for protected operators and q-hypergeometric identities to $\mathrm{n}=1$ dual theories. Nucl. Phys. B 818, 137-178. arXiv:0801.4947 (2009)

170. Rastelli, L., Razamat, S.S.: The Superconformal Index of Theories of Class $\mathcal{S}$. In: Teschner, J. (ed.) New Dualities of Supersymmetric Gauge Theories, pp. 261-305. arXiv:1412.7131 (2016)

171. Yoshida, Y.: Factorization of $4 d \mathrm{~N}=1$ Superconformal Index. arXiv:1403.0891 (2014)

172. Peelaers, W.: Higgs branch localization of $\mathcal{N}=1$ theories on $\mathrm{S}^{3} \times \mathrm{S}^{1}$. JHEP $\mathbf{0 8}, 060$. arXiv:1403.2711 (2014)

173. Chen, H.-Y., Chen, H.-Y.: Heterotic surface defects and dualities from 2d/4d indices. JHEP. 10, 004 arXiv:1407.4587 (2014)

174. Razamat, S.S.M. Yamazaki: S-duality and the $\mathrm{N}=2$ lens space index. JHEP 10, 048. arXiv:1306.1543 (2013)

175. Closset, C., Shamir, I.: The $\mathcal{N}=1$ chiral multiplet on $T^{2} \times S^{2}$ and supersymmetric localization. JHEP 03, 040. arXiv:1311.2430 (2014)

176. Assel, B., Cassani, D., Martelli, D.: Localization on Hopf surfaces. JHEP 08, 123. arXiv:1405.5144 (2014)

177. Nishioka, T., Yaakov, I.: Generalized indices for $\mathcal{N}=1$ theories in four-dimensions. JHEP 12, 150. arXiv:1407.8520 (2014)

178. Honda, M., Yoshida, Y.: Supersymmetric index on $T^{2} \times S^{2}$ and elliptic genus. arXiv:1504.04355 (2015)

179. Gadde, A., Razamat, S.S., Willett, B.: On the reduction of $4 \mathrm{~d} \mathcal{N}=1$ theories on $\mathbb{S}^{2}$. JHEP 11, 163. arXiv: 1506.08795 (2015)

180. Gadde, A., Rastelli, L., Razamat, S.S., Yan, W.: The 4d superconformal index from q-deformed $2 \mathrm{~d}$ Yang-Mills. Phys. Rev. Lett. 106, 241602 arXiv:1104.3850 (2011)

181. Gaiotto, D., Rastelli, L., Razamat, S.S.: Bootstrapping the superconformal index with surface defects. JHEP 01, 022. arXiv:1207.3577 (2013)

182. Razamat, S.S.: On the $\mathcal{N}=2$ superconformal index and eigenfunctions of the elliptic RS model. Lett. Math. Phys. 104, 673-690. arXiv:1309.0278 (2014)

183. Faddeev, L.D.: Modular double of quantum group. Math. Phys. Stud. 21, 149-156. arXiv:math/9912078 (2000)

184. Kharchev, S., Lebedev, D., Semenov-Tian-Shansky, M.: Unitary representations of u(q) (sl(2, r)), the modular double, and the multiparticle q deformed toda chains. Commun. Math. Phys. 225, 573609. arXiv:hep-th/0102180 (2002)

185. Narukawa, A.: The modular properties and the integral representations of the multiple elliptic gamma functions. Adv. Math. 189(2), 247-267. arXiv:math/0306164 (2004) 